\title{
Colonization pattern of crop plants by endophytic fungi
}

\author{
Dissertation \\ to obtain the $\mathrm{Ph}$. D. degree \\ in the International Ph. D. Program for Agricultural Sciences in Goettingen (IPAG) \\ at the Faculty of Agricultural Sciences, \\ Georg-August-University Göttingen, Germany
}

Presented by

Leilei Zhang

Born in Shandong, P. R. China

Göttingen, May 2014 
D7

1. Name of supervisor: Prof. Dr. Stefan Vidal

2. Name of co-supervisor: Prof. Dr. Petr Karlovsky

Date of dissertation: 


\section{Table of contents}

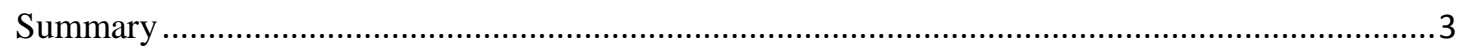

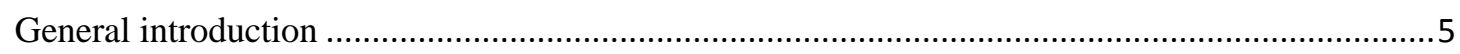

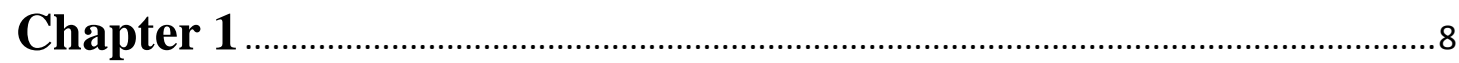

Comparison of different methods to introduce Beauveria bassiana as endophyte in cabbage..........8

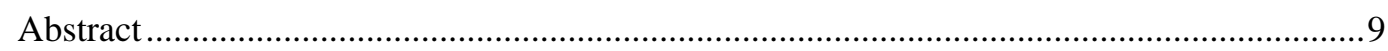

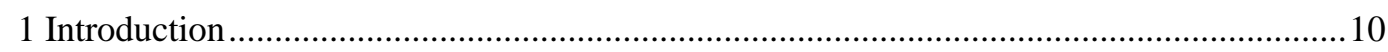

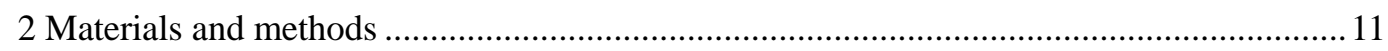

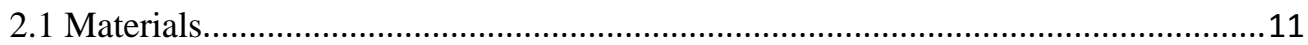

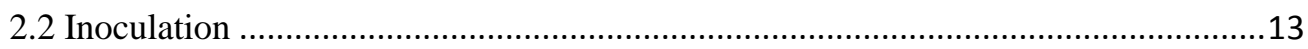

2.3 Selective medium re-isolation........................................................................14

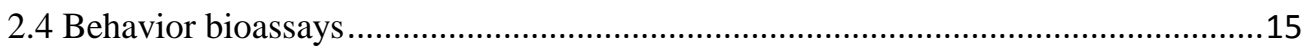

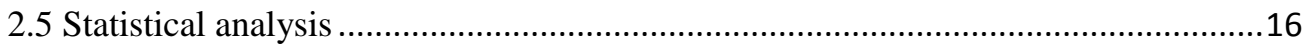

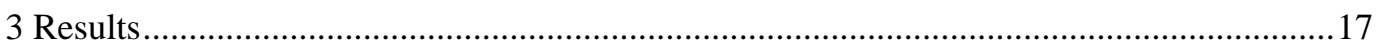

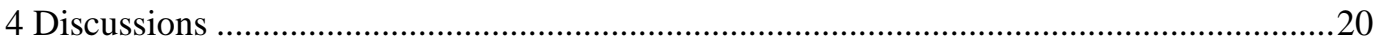

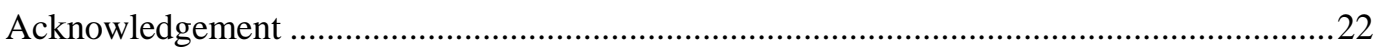

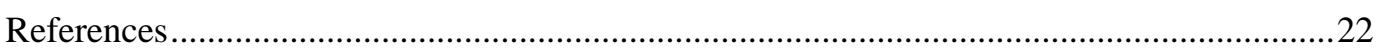

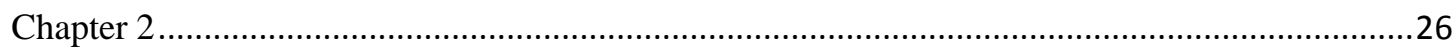

Endophytic establishment of Beauveria bassiana and Trichoderma harzianum in cabbage ...........26

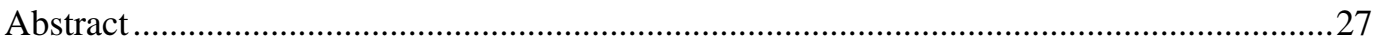

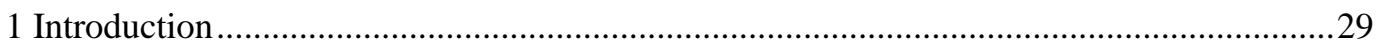

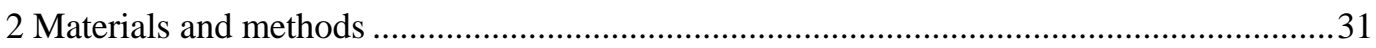

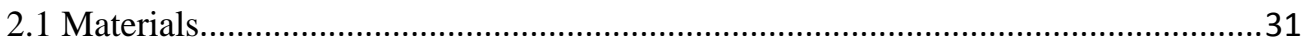

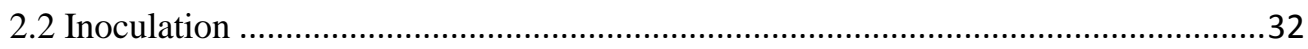

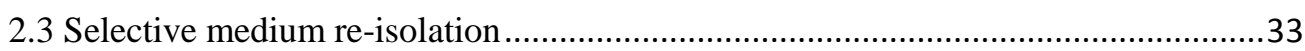

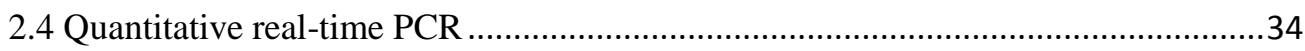

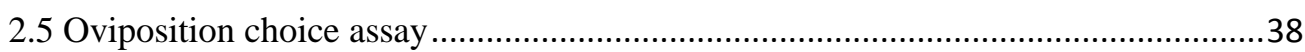

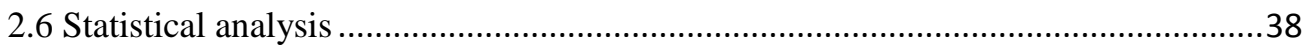

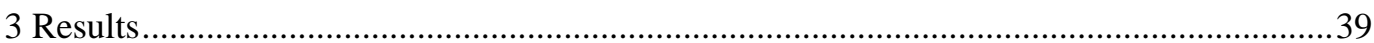

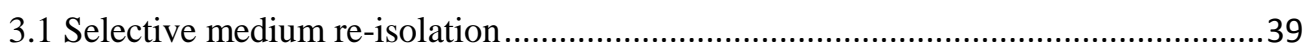

3.2 Real time PCR quantification of fungal colonization ............................................40

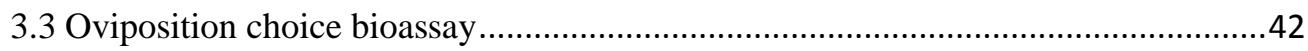

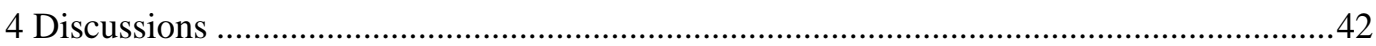

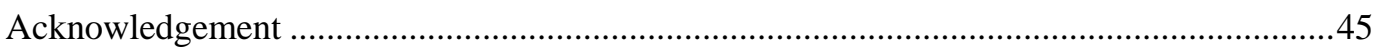

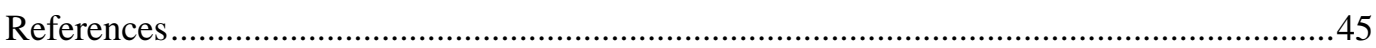

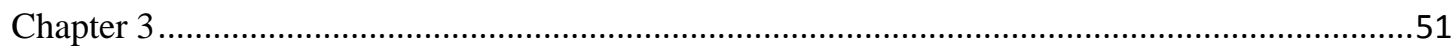

Trichoderma harzianum colonization triggers plant systemic defense against herbivores.............51

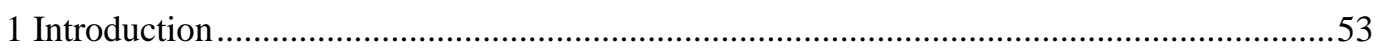

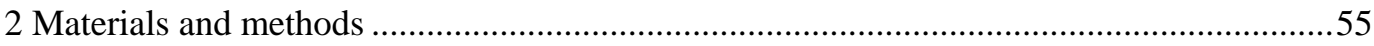

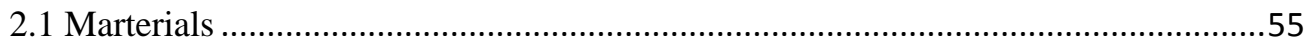

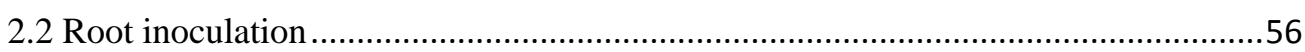


2.3 Selective medium re-isolation ....................................................................5

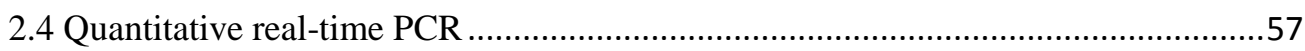

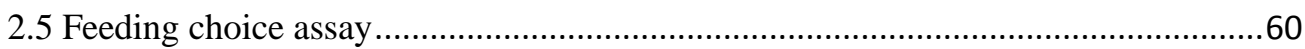

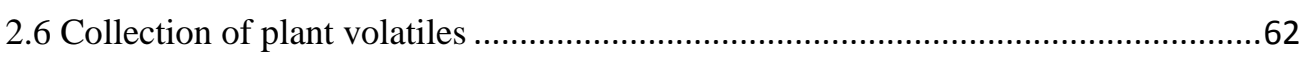

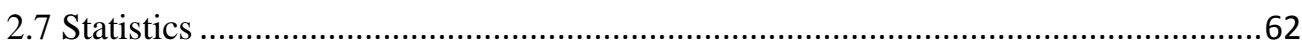

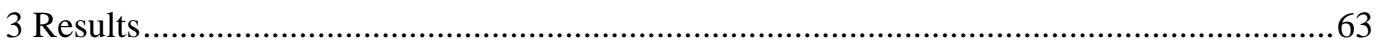

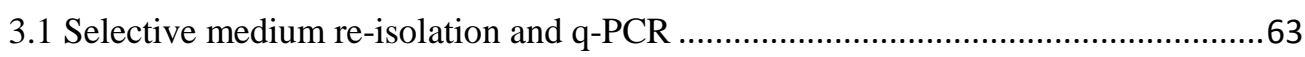

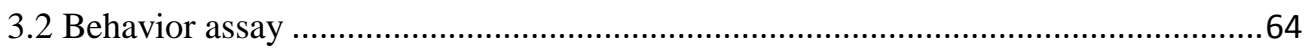

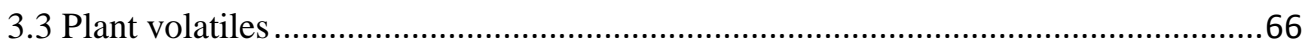

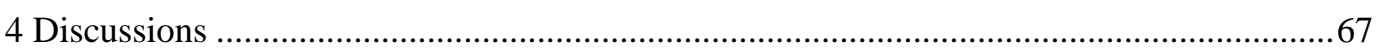

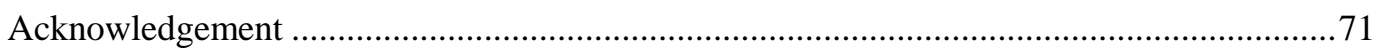

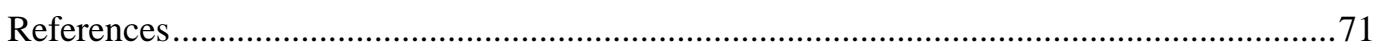

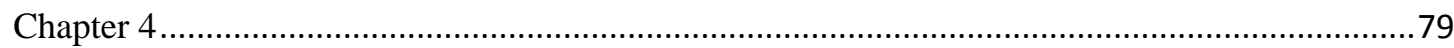

Endophytic colonization of faba bean Vicia faba by two beneficial fungi: Beauveria bassiana and

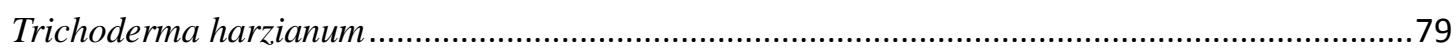

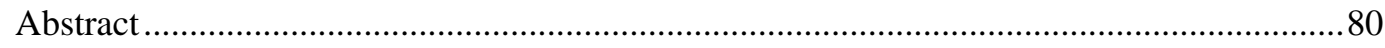

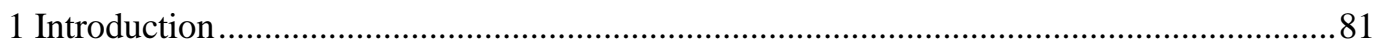

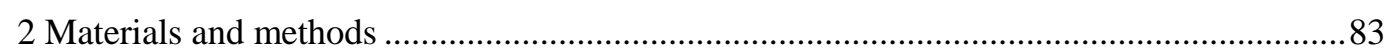

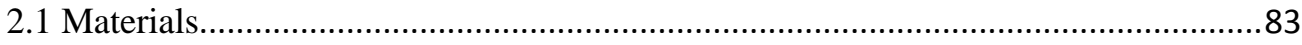

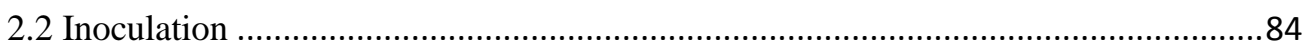

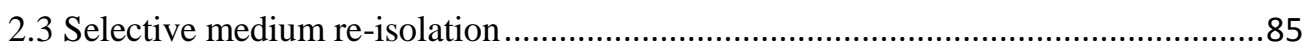

2.4 Quantitative real-time PCR ...................................................................... 86

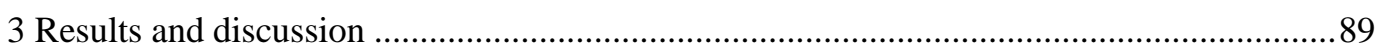

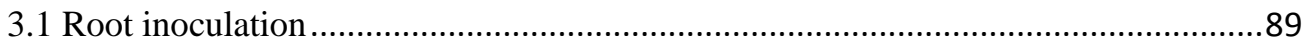

3.2 Seed inoculation ....................................................................................... 91

3.3 Comparison of the colonization of different fungi and different inoculation methods

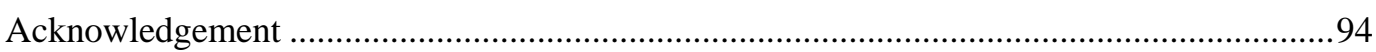

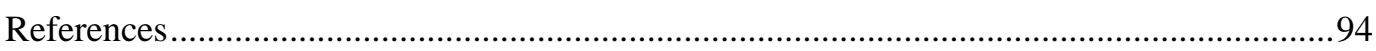

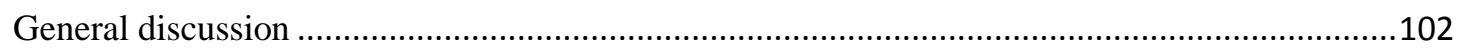

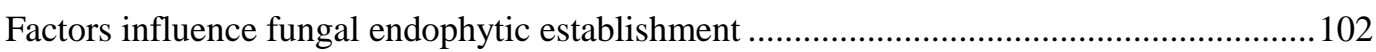

Inoculation methods and fungal species....................................................... 102

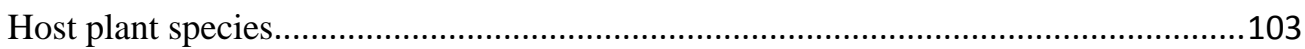

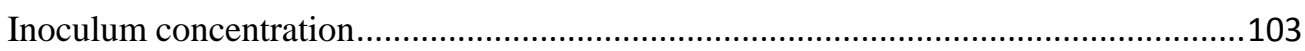

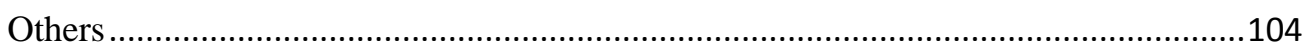

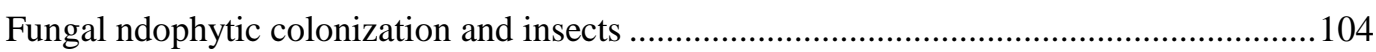

References cited in general introduction and discussion.....................................................106

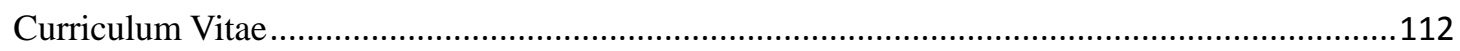

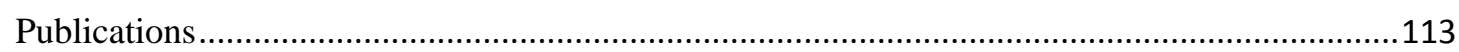

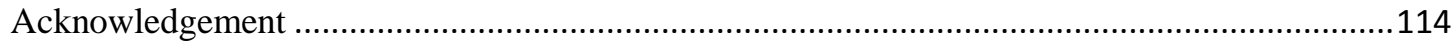




\section{Summary}

Endophytes are fungi or bacteria occurring inside plant tissues without causing any apparent symptoms in the host plants. Fungal endophytes are quite common in nature; some of them are able to work as bio-control agents to defend insects, nematodes and plant pathogens. As endophytic fungi can continuously be presented and expressed throughout the crop cycle. Such an endophytic expression of the entomopathogens within the plant system is expected to be more advantageous than external application of bio-agents. Fungi Beauveria bassiana (strain ATP01, ATP02, Bb03032, EABb04/01-tip and Naturalis) and Trichoderma harzianum (strain T39 and Tu) have been already proved and well-studied as endophytes in host plants to work against either herbivores or plant pathogens.

This study aimed at introducing B. bassiana and T. harzianum to host plant cabbage and faba bean as endophytes. The distribution of the endophytic fungi was investigated by selective medium re-isolation and q-PCR with the comparison ofdifferent inoculation methods.. Bioassays were conducted to test the influence of the fungal endophytic colonization on herbivores.

Main findings:

Both B. bassiana and T. harzianum were able to establish in the host plants as endophytes. By different inoculation methods, various colonization patterns were found where $B$. bassiana had the best fungal establishment in leaf inoculated treatments while T. harzianum had the better performance in below ground inoculated trials (root inoculation and seed inoculation).

With root and seed inoculation, both B. bassiana and T. harzianum had a higher colonization level in faba bean compared with cabbage. 
The existence of endophytic fungi influenced the development and behavior of the diamondback moth (DBM) Plutella xylostella. DBM larvae feeding on detached $B$. bassiana (strain ATP02) inoculated leaf had a slower development and a lower body weight. In the dual-choice oviposition choice experiments the DBM significantly preferred control plants to B. bassiana inoculated plants. At a high colonization level, endophytic T. harzianum was able to affect both the feeding choice and oviposition choice in the dual-choice bioassays, although T. harzianum could not kill DBM larvae directly and the endophytic $T$. harzianum also did not affect the DBM larvae development significantly.

The endophytic establishment of $T$. harzianum strongly influenced the volatile emission. The green volatile compounds content decreased while the percentage composition of terpenes increased in T. harzianum inoculated treatments. 


\section{General introduction}

The term “endophyte” was first described by German botanist Anton de Bary in 1884 (De Bary 1884), and later was specifically defined as fungi or bacteria occurring inside plant tissues without causing any apparent symptoms in the host plants by Wilson (1995). The potential importance of endophytic fungi was not recognized until 1970s, when the endophytes of pasture grasses in the family Clavicipitacheae were found to be toxic to cattle (Bacon et al. 1975, Bacon et al. 1977, Porras-Alfaro and Bayman 2011). Currently, as fungal endophytes were found in almost all plant species, their ecological role has been well studied (Stone et al. 2004). Studies demonstrated that some of the endophytes are able to promote plant growth, improve resistance to environment stresses, and work against insects, nematodes and plant pathogens (Vega et al. 2008, Porras-Alfaro and Bayman 2011, Biswas et al. 2012). The defense of endophytic fungi against pests and herbivores is accomplished by antagonism, including producing fungal metabolites, competition or parasitism and the induction of host defenses and stimulation of host growth vigor (Alabouvette et al. 2009, Porras-Alfaro and Bayman 2011). However, the mechanism of how these microbial communities affect plant health and function is not always clear.

As endophytes are able to colonize the host tissues internally and are present inside the host continuously, they were expected to be more advantageous than traditional microbial control agents (Azevedo et al. 2000). Since the ecological function of endophytes in plant has been well recognized, to date, more studies have been conducted focusing on artificially introduced beneficial fungi to host plants. In the current study, the abilities of endophytic colonization of two famous fungi Beauveria bassiana (Balsamo) Vuillemin (Ascomycota: Hypocreales) and Trichoderma harzianum Rifai (Ascomycota: Hypocreales) were investigated.

Being one of the most famous entomopathogen, $B$. bassiana was shown to be able to establish either naturally or by artificial inoculation in maize (Bing and Lewis 
1991), potato (Jones 1994), cocoa (Posada and Vega 2005), coffee (Posada et al. 2007), banana (Akello et al. 2007b), date palm (Gomez-Vidal et al. 2006), sorghum (Tefera and Vidal 2009), opium poppy (Quesada-Moraga et al. 2009), cotton, pumpkin, wheat (Gurulingappa et al. 2010), Pinus radiate (Reay et al. 2010), jute (Biswas et al. 2012) and common bean (Parsa et al. 2013). Meanwhile, the capability of endophytic B. bassiana in defending the herbivores was also proven in many cases. Studies showed that endophytic B. bassiana was able to reduce the tunneling of European corn borer larvae Ostrinia nubilalis in maize (Bing and Lewis 1991), suppress the stem-borer Sesamia calamistis in maize (Cherry et al. 2004); reduce the population of banana weevil Cosmopolites sordidus (Akello et al. 2008); control the stem borer Chilo partellus in millet crop plant (Sorghum bicolor) (Reddy et al. 2009); and provide systemic protection against damage by poppy stem gall wasp Iraella luteipes in opium poppy (Quesada-Moraga et al. 2009).

Free-living fungus T. harzianum is famous for its capacity to control plant pathogens (Harman et al. 2004). Moreover, Trichoderma spp. also improves photosynthetic efficiency and increases nutrient use efficiency in plants, which can result in the promotion of the plant growth and greater yields in colonized plants (Shoresh et al. 2010). Currently, there is no doubt about the capacity of T. harzianum to act as a beneficial fungus to protect crops from phytopathogens, but there are different arguments about whether T. harzianum can act pathogenic against insects. For instance, T. harzianum was able to control damping off disease of beans Phaseolus vulgaris L. caused by Fusarium solani and Rhizoctonia solani (Abd-El-Khair et al. 2010) and to eliminate chocolate spot disease in faba bean caused by Botrytis fabae Sard. or B. cinerea Pers. (Saber et al. 2009, Abd El-Rahman and Mohamed 2014). Besides the evidence we listed in the introduction that T. harzianum has a positive effect on herbivore control, studies also showed that the presence of some T. harzianum strains has no direct effect to the insect. Studies showed that $T$. harzianum was able to lead to a high mortality in Oncopeltus fasciatus (Santamarina et al. 2002); significantly reduces spruce beetle survival and reproduction (Cardoza et 
al. 2006); induces a higher mortality in Tenebrio molitor larvae (Shakeri and Foster 2007) and results in a significant decrease of aphid Myzus persicae survival (Ganassi et al. 2009). On the other side, some studies also illustrated that $T$. harzianum was not harmful to termites Coptotermes formosanus, honey bees Apis mellifera, bumble bees Bombus terrestris and dusky sap beetle Carpophilus lugubris (Vega et al. 1995, Brownold et al. 1997, Kovach et al. 2000, Van der Steen et al. 2004, Jayasimha and Henderson 2007, Mommaerts et al. 2008, Albano et al. 2009).

Species and cultivar of the host plants, species and strain of endophytes, concentration of the inoculum, age and growing conditions of the host plants are considered as the major factors which influence the establishment of the artificial introduce of endophytes (Kessler et al. 2003, Tefera and Vidal 2009, Parsa et al. 2013). In this study, different inoculation methods (leaf, root, seed, shoot and stem inoculations) were used to introduce $B$. bassiana and $T$. harzianum to the host plant cabbage or faba bean; the fungal colonization status in different tissues of the host plant was investigated by selective medium re-isolation and quantitative PCR. Moreover, the influence of the fungi endophytic colonization on herbivores was also studied via feeding and oviposition bioassays. The most notorious pest diamondback moth (DBM) Plutella xylostella were used as the tested insects in the bioassays. 


\title{
Chapter 1
}

\section{Comparison of different methods to introduce Beauveria bassiana as}

\author{
endophyte in cabbage
}

Leilei Zhang and Stefan Vidal

Georg-August-University Goettingen

Department of Crop Sciences

Section of Agricultural Entomology

Grisebachstrasse 6

37077 Goettingen Germany 


\begin{abstract}
Five different Beauveria bassiana strains (ATP01, ATP02, Bb03032, EABb04/01-tip and Naturalis) were tested for their capability to colonize cabbage plants with different inoculation methods. All five strains were able to colonize the complete plant tissue with a leaf, shoot or root inoculation. The colonization rates in the stems were over $50 \%$ for all the strains with a leaf inoculation and decreased to 5\%-30\% with a shoot and root inoculation. The newer emerged leaves always had a lower fungal colonization most likely due to the slower growth of the endophytic $B$. bassiana than the host plant. Feeding and ovipositing bioassays were conducted to test whether the colonization of the B. bassiana could affect the performance of the diamondback moth (DBM) Plutella. xylostella. DBM larvae fed by ATP02 inoculated leaves had a significant weight loss and needed a significant longer time to pupate and emerge. Oviposition bioassay from the leaf inoculated plants revealed that DBM adults had a significant preference for control plants to inoculated plants. These results indicate that the endophytic $B$. bassiana can be established as an effective bio-control agent.
\end{abstract}

Key words: Beauveria bassiana, inoculation, colonization, endophyte, entomopathogenic fungi 


\section{Introduction}

Fungal endophytes live within host plant tissues without causing any visible symptoms of diseases (Wilson 1995) and are very common in nature. Vega et al. and references therein described that some of them have the ability to work against insects, nematodes and plant pathogens (2008). The use of endophytic entomopathogenic fungi as new bio-control agents has been widely studied in recent years (Vega et al. 2008, Biswas et al. 2012). As endophytic entomopathogens can continuously exist and their activity expressed throughout the crop cycle, endophytic colonization of entomopathogens within the plant system is expected to be more advantageous than external application (Biswas et al. 2012).

The most famous entomopathogen, Beauveria bassiana has been widely studied for its ability to colonize naturally as well as in response to artificial inoculation in maize (Bing and Lewis 1991), potato (Jones 1994), cocoa (Posada and Vega 2005), coffee (Posada et al. 2007), banana (Akello et al. 2007b), date palm (Gomez-Vidal et al. 2006), sorghum (Tefera and Vidal 2009), opium poppy (Quesada-Moraga et al. 2009), cotton, pumpkin, wheat (Gurulingappa et al. 2010), Pinus radiate (Reay et al. 2010), jute (Biswas et al. 2012) and common bean (Parsa et al. 2013). The endophytic B. bassiana has been demonstrated to suppress many pest species by significantly reducing the tunneling of European corn borer larvae (Ostrinia nubilalis) in maize (Bing and Lewis 1991) and successfully suppressing the stem-borer (Sesamia calamistis) in maize (Cherry et al. 2004); the banana weevil (Cosmopolites sordidus) (Akello et al. 2008), the stem borer Chilo partellus in millet crop plant (Sorghum bicolor) (Reddy et al. 2009) or providing systemic protection against damage by the poppy stem gall wasp, Iraella luteipes in opium poppy (Quesada-Moraga et al. 2009).

Different inoculation methods including soil drenching (Tefera and Vidal 2009), seed coating and immersion (Brownbridge et al. 2012), root and rhizome immersion (Akello et al. 2009, Posada et al. 2010), stem injection (Posada et al. 2007) foliar spray (Tefera \& Vidal, 2009 and flower spray (Posada et al. 2010) have been used to 
introduce B. bassiana as endophytes into host plants.

The establishment status of $B$. bassiana in the host plants is related to both the inoculation methods and fungal strains. In this study, we tried to introduce five different B. bassiana strains (ATP 01, ATP 02, Bb03032, EABb04/01-tip (EABb04), and commercial strain Naturalis) to host cabbage plant Brassica oleracea var. capitata L, using three different inoculation methods (leaf spray, shoot brush and root immersion). This study aimed to compare these inoculation methods and the performance of different strains; the $B$. bassiana establishment was investigated according to the growth of host plants in leaf and shoot inoculation treatments. Feeding and ovipostion bioassays were also conducted to test whether the colonization of the $B$. bassiana influences the development and oviposition behavior of the herbivore diamondback moth (DBM), Plutella xylostella.

\section{Materials and methods}

\subsection{Materials}

\subsubsection{Fungi}

Five strains of $B$. bassiana were used in the experiments (ATP 01, ATP 02, Bb03032, EABb04 and Naturalis). The origins of the strains are listed in Table 1.

Fungal conidia were harvested from 3-week-old PDA (potato dextrose agar) cultures. Fungal conidial suspensions were prepared in sterile $0.1 \%$ Tween 20 solution and the conidia concentration was adjusted to $1 \times 10^{8}$ conidia $/ \mathrm{ml}$ or $2 \times 10^{7}$ conidia $/ \mathrm{ml}$ using an improved Neubauer hemocytometer. The germination of all the fungal strains was checked by PDA medium to ensure the vitality of the fungal spores (Posada and Vega 2005). 
Table 1 Beauveria bassiana strains used in the experiments

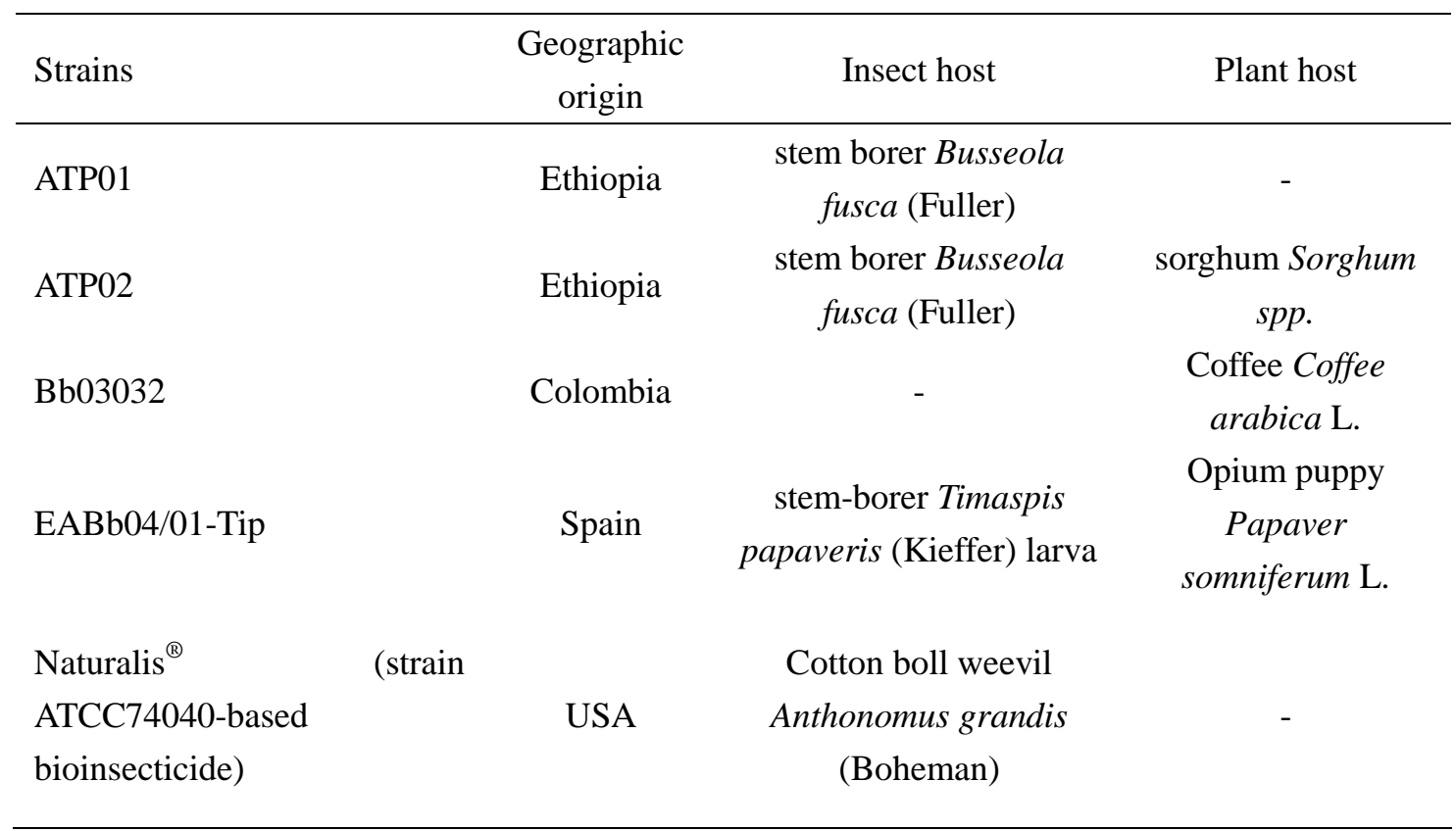

\subsubsection{Host plant}

Cabbage plants, Brassica oleracea var. capitata L. (flat-leaf cultivar: Furchenkohl; Norddeutsche Pflanzenzucht Hans-Georg Lembke KG) were grown in seedling trays from seeds in a greenhouse chamber $\left(24 \pm 3^{\circ} \mathrm{C}\right.$; $80 \%$ relative humidity; 16L: 8D photoperiod). 10-day-old seedlings were transplanted into $11 \mathrm{~cm}$ diameter plastic pots with non-sterile soil (Fruhstorfer Erde Typ T, Hawita Gruppe GmbH, Vechta, Germany) and sand mixture (3:1 volume). Plants were grown in the chamber, irrigated regularly and fertilized once a week until needed (fertilizer: Hakaphos 2g/L (15\% N, 11\% $\mathrm{P}_{2} \mathrm{O}_{5}, 15 \% \mathrm{~K}_{2} \mathrm{O}, 1 \% \mathrm{Mg}, 0.1 \% \mathrm{Fe}, 0.1 \% \mathrm{Mn}, 0.04 \% \mathrm{Cu}, 0.025 \% \mathrm{~B}$ and $0.005 \% \mathrm{Mo}))$.

\subsubsection{Insect}

The diamondback moths (DBM) used in the bioassays were collected from a laboratory culture which has been kept in a cage $(90 \mathrm{~cm} \times 50 \mathrm{~cm} \times 50 \mathrm{~cm})$ in a climate controlled insectary $\left(18 \pm 2{ }^{\circ} \mathrm{C}, 70 \pm 5 \%\right.$ relative humidity; L16:D8 photoperiod), fed with cabbage plants (cultivar: Furchenkohl) until needed. 


\subsection{Inoculation}

Leaf inoculation: 3-week-old cabbage plants (growth stage: 5 leaves) were used for inoculation. The leaf inoculation was done on the newly-emerging leaves ( $6^{\text {th }}$ leaf) by spraying on its upper and lower surface with a $1 \times 10^{8}$ conidia/ml fungal suspension. The inoculation was handled with in a plastic bag in order to avoid the contamination on the other leaves. Approximate $3 \mathrm{ml}$ fungal suspension was applied on each leaf using a hand sprayer. Instead of fungal suspension, sterile water with $0.1 \%$ Tween 20 was used in control treatment. The plastic bag was maintained on the plants for $24 \mathrm{~h}$ to maintain a high humidity which could enhance the fungal spore germination.

Shoot inoculation: Sterile camel-hair brushes were dipped into the $1 \times 10^{8}$ conidia/ml suspensions; the brush (carried with B. bassiana spores) was used to attach the spores onto the shoot ( $3 \mathrm{~cm}$ long stem area from top) of a 3-week-old cabbage plant by carefully touching the shoot with the brush. As the colonization status would be checked in next six newer expanding leaves, metal rings were used to mark the first newly-expand leaves. Sterile water with $0.1 \%$ Tween 20 was used in control application.

Root inoculation: Cabbage seedlings were gently removed from the pots two weeks after transplanting, and the soil was washed off under tap water. During root washing specific care was taken to not damage any roots. The inoculation was performed by immersing the root of the seedling into $2 \times 10^{7}$ conidia/ml B. bassiana spore suspension or sterile water (with $0.1 \%$ Tween 20 for the control treatment) for 30 minutes. In the next step, the plants were planted into new $11 \mathrm{~cm}$ diameter pots and irrigated well.

All the plants were kept in the greenhouse chamber, irrigated regularly and fertilized once a week (fertilizer: Hakaphos 2g/L). 


\subsection{Selective medium re-isolation}

Leaf and shoot inoculation: The colonization rate of the newly-emerged leaves were tested at different leaf growth stages using $B$. bassiana selective medium (2\% oatmeal infusion, 2\% agar, $550 \mathrm{mg} / \mathrm{L}$ dodine, $5 \mathrm{mg} / \mathrm{L}$ chloramphenicol $5 \mathrm{mg} / \mathrm{L}$ crystal violet) (Chase et al. 1986). After the inoculation, the colonization rates in the newly-emerged leaves were tested six times at 6-day intervals. For each leaf, four leaf discs were obtained directly from the leaves of the cabbage plants with a sterile cork borer (diameter: $13 \mathrm{~mm}$ ). For the first three checked leaves, a second check was processed six days later on the same leaf on the opposite side of the first sampled sites. The $13 \mathrm{~mm}$ diameter leaf discs were surface sterilized with 70\% ethanol 1minutes, $2 \%$ $\mathrm{NaClO}$ three minutes, and rinsed with sterile water for three times. The samples were dried in a clean bench. As the sterilization procedure might deactivate the fungal mycelium grown close to the edge of the leaf discs, a smaller sterile cork borer (diameter: $8 \mathrm{~mm}$ ) was used to cut off the middle of the original leaf discs. The mycelium in this section was considered to be not affected. In the leaf inoculated trials, the colonization status of the inoculated leaves was checked three days after the inoculation by sampling four leaf discs from each leaf. With four leaf discs per plant and ten plants per treatment, a total number of forty leaf discs were obtained for each treatment. For both leaf and stem inoculations, the stem samples were obtained at the time of harvest. Four stem segments (five mm, average distributed from top to bottom of the stem) were obtained from each stem.

Root inoculation: The root inoculated plants were only checked one time at four weeks after the inoculation by the time of harvest. Three leaves per treatment (newly-expanded, medium, and oldest leaves) and four leaf discs per leaf were sampled and surfaced sterilized with the same sterilization method as leaf and shoot inoculations. After surface sterilization, a sterile $8 \mathrm{~mm}$ diameter sterile cork borer was used to obtain the samples as described with the leaf and shoot inoculation. The 
colonization status of stems and roots were also analyzed: a single stem was cut into twelve segments and placed on selective medium after surface sterilization; three 2 cm long root segments per plant were obtained and placed on selective medium after surface sterilization. Only three B. bassiana strains (Bb3032, EABb04, and Naturalis) were used in the root inoculation. There were ten replicates per treatment.

All petri dishes with the plant sample on the selective medium were sealed with parafilm (Pechiney Plastic Packaging. Chicago Il) and incubated at $25^{\circ} \mathrm{C}$ in the incubator for 3 weeks, by then the fungal existence was examined visually for fungal growth. Fungal growth was characterized as $B$. bassiana based on white dense mycelia, becoming cream to pale yellow at the edge (Humber 1997). For each treatment, the fungal percent colonization was calculated following the Petrini (1991) formula: colonization rate $=$ number of plant samples showing $B$. bassiana outgrowth divided by the total number of incubated plant samples $\times 100 \%$.

\subsection{Behavior bioassays}

As leaf inoculation was more efficient than shoot and root inoculation. Thus leaf inoculated plants were used in the bioassay test.

\section{Detached leaf feeding bioassay}

B. bassiana ATP02 $\left(1 \times 10^{8}\right.$ conidia/ml $)$ and sterilized water $(0.1 \%$ Tween 20$)$ leaf inoculated plants were used in the experiment. Second-instar DBM larvae were used in this bioassay. The experiment was conducted in $9 \mathrm{~cm}$ diameter petri-dishes in a climate chamber with $25^{\circ} \mathrm{C}, 60 \%$ relative humidity and 16 -hours photoperiod. Filter paper was used to cover the bottom of the petri dish, and then a single larva was kept in each petri dish. A $4 \mathrm{~cm}$ diameter leaf discs from the surface sterilized detached ATP02 sprayed leaf or water sprayed leaf (at the same age) were used to feed the insects. Newly obtained leaf discs were used to replace the old ones every day. The weight of the insects was recorded every day until the first adult emerged. 10 
replicates were used in each treatment.

\section{Oviposition choice assay:}

Insect rearing cages (size: $90 \mathrm{~cm} \times 50 \mathrm{~cm} \times 50 \mathrm{~cm}$ ) were used in the oviposition bioassay. Fresh pupae from lab culture were collected into a plastic box $(18 \mathrm{~cm} \times 15$ $\mathrm{cm} \times 5 \mathrm{~cm}$ ). After five days, five pairs of newly emerged male and female DBMs were placed in a test tube $(1.7 \mathrm{~cm}$ diameter $\times 10.5 \mathrm{~cm}$ length $)$ and closed off with a lid. Two cabbage plants were placed on opposite sides of the rearing cage. One plant has been inoculated with strain EABb04 or Naturalis with leaf inoculation-method; the second plant served as a $B$. bassiana-free control. The lid of the test tube with the DBM was removed and the test tube placed in the half way between the plants in the cage. To avoid the influence of light on DBM behavior, the cages were left in darkness for 48 hours. In a pre-experiment, the DBM was found to have a significant preference to oviposit on parafilm (Pechiney Plastic Packaging. Chicago Il), thus in this experiment, to enhance the oviposition performance of DBM, parafilm stripes were used to cover all the stems surface of the tested plants to form the oviposition sites. The cabbage plants were then removed from the cage and the number of the eggs oviposited on each plant (including the pots, stems, leaves) counted. Ten cages for each treatment were applied in the assay.

\subsection{Statistical analysis}

Raw data were checked for normality and homogeneity of variance using the Shapiro-Wilk test. Repeat measure ANOVA was used to test whether the larval weights between the two groups in the feeding bioassay were significant. One way ANOVA was used to compare the emerged and pupated days between two groups. The overall number of eggs for each treatment was analyzed by goodness-of-fit test (SAS 9.3) 


\section{Results}

Spore germination rates of all $B$. bassiana strains used in the experiment were above $90 \%$. No B. bassiana was observed in the selective medium used to grow the third time rinsing water which indicated the sterilization process was sufficient to exclude the leaf surface $B$. bassiana. Therefore, outgrowth of $B$. bassiana from surface-sterilized leaf discs originated from endophytic colonization of the plant tissues by the fungus. Besides the artificially inoculated B. bassiana, according to the morphology of the fungi mycelium and spores, some other fungi were also found to occur as natural endophytes in cabbage, those fungi included: Acremonium strictum Aspergillus spp., Chaetomium globosum, Penicillium spp. and Trichoderma harzianum.

Table 2 Colonization rates (\%) of the newly-expanded leaves of $\boldsymbol{B}$. bassiana leaf inoculated cabbage

\begin{tabular}{lcccccccccccc}
\hline \multirow{2}{*}{ Strain } & \multirow{2}{*}{ Inoculated $^{\mathrm{a}}$} & \multicolumn{2}{c}{ U1 $^{\mathrm{b}}$} & \multicolumn{1}{c}{ U2 } & \multicolumn{3}{c}{ U3 } & \multirow{2}{*}{ U4 } & \multirow{2}{*}{ U5 } & U6 & Stem \\
\cline { 3 - 10 } & & U1A $^{\mathrm{c}}$ & U1B & U2A & U2B & U3A & U3B & & & & \\
\hline Control & 0 & 0 & 0 & 0 & 0 & 0 & 0 & 0 & 0 & 0 & 0 \\
ATP01 & 80.0 & 17.5 & 15.0 & 10.0 & 5.0 & 10.0 & 7.5 & 5.0 & 2.5 & 0.0 & 70.0 \\
ATP02 & 75.0 & 30.0 & 17.5 & 15.0 & 15.0 & 12.5 & 10.0 & 15.0 & 7.5 & 5.0 & 52.5 \\
EABb04 & 90.0 & 17.5 & 30.0 & 17.5 & 5.0 & 2.5 & 2.5 & 2.5 & 7.5 & 5.0 & 55.0 \\
Bb03032 & 47.5 & 2.5 & 7.5 & 7.5 & 5.0 & 5.0 & 5.0 & 5.0 & 2.5 & 2.5 & 50.0 \\
Naturalis & 85.0 & 17.5 & 10.0 & 12.5 & 7.5 & 7.5 & 2.5 & 2.5 & 2.5 & 2.5 & 55.0 \\
\hline
\end{tabular}

Ten plants ( $=10$ replicates) were used in each treatment; ${ }^{\text {a }}$ : inoculated leaves: checked three days after the inoculation; ${ }^{\mathrm{b}}$ : U1-U6 : newly expanded leaves checked 6, 12, 18, 24, 30, 36 days after inoculation; ' : U1B, U2B, and U3B checked 6 days after U1A, U2A, U3A, respectively

B. bassiana could colonize the cabbage leaves and stems with a leaf inoculation successfully. The inoculated leaves, examined three days after inoculation, were successfully colonized by B. bassinana with the highest (90\%) by strain EABb04 and the lowest colonization rate by strain Bb03032 (47.5\%). The colonization rates of newer leaves, checked at subsequent sampling dates, were lower at a more advanced growth stage; by the sixth sampling date, strain ATP01 was not recovered in the examined leaves. The analysis of the same leaf at 6-days interval showed that in most 
cases the recovery rates did not increase 6 days after inoculation. All the strains had colonization rates higher than $50 \%$ in the stem which indicated that, all tested $B$. bassiana strains were able to colonize the host plants systemically with a leaf inoculation.

A similar pattern in leaf inoculated treatments than in shoot inoculated cabbage was found but with lower colonization rates (Table 3). With shoot inoculation, however, the colonization rates of all the strains remained at lower levels. The highest recovery rates for all the strains were from the first expanded leaves (22.5\% ATP02 in the second check of first expanded leaf). As the plant grew, the colonization rates of all the strains were getting as low as zero from the fourth leaves. The recovery rates of B .bassiana were also much lower a stem than a leaf inoculation.

Table 3 Colonization rates (\%) of the newly-expanded leaves of $B$. bassiana shoot inoculated cabbage

\begin{tabular}{|c|c|c|c|c|c|c|c|c|c|c|}
\hline \multirow{2}{*}{ Strain } & \multicolumn{2}{|c|}{$\mathrm{U} 1^{\mathrm{a}}$} & \multicolumn{2}{|c|}{$\mathrm{U} 2$} & \multicolumn{2}{|c|}{ U3 } & \multirow{2}{*}{$\mathrm{U} 4$} & \multirow{2}{*}{ U5 } & \multirow{2}{*}{ U6 } & \multirow{2}{*}{ Stem } \\
\hline & $\mathrm{U}^{\mathrm{A}} \mathrm{A}^{\mathrm{b}}$ & U1B & U2A & $\mathrm{U} 2 \mathrm{~B}$ & U3A & U3B & & & & \\
\hline Control & 0 & 0 & 0 & 0 & 0 & 0 & 0 & 0 & 0 & 0 \\
\hline АТР01 & 20.0 & 17.5 & 12.5 & 10.0 & 5.0 & 7.5 & 2.5 & 0.0 & 2.5 & 10.0 \\
\hline АТР02 & 15.0 & 22.5 & 17.5 & 5.0 & 2.5 & 2.5 & 2.5 & 0.0 & 0.0 & 5.0 \\
\hline EABb04 & 10.0 & 7.5 & 12.5 & 5.0 & 2.5 & 2.5 & 0.0 & 2.5 & 2.5 & 12.5 \\
\hline Bb3032 & 20.0 & 10.0 & 15.0 & 12.5 & 10.0 & 7.5 & 12.5 & 5.0 & 0.0 & 7.5 \\
\hline Naturalis & 10.0 & 10.0 & 10.0 & 2.5 & 0.0 & 5.0 & 0.0 & 0.0 & 0.0 & 5.0 \\
\hline
\end{tabular}

Ten plants (= 10 replicates) were used in each treatment; ${ }^{\mathrm{a}}$ : U1-U6 : newly expanded leaves checked 6, 12, 18, 24, 30, 36 days after inoculation; ':U1B, U2B, and U3B checked 6 days after U1A, U2A, U3A, respectively

In the root inoculation trials, although the inoculation was done in the roots, the root system was not fully-established with the B.bassiana endophyte. Strain EABb04 and Naturalis had 30\% colonization rates while strain Bb03032 was slightly lower than $20 \%$ (Table 4). In the stems, the colonization rates were lower than the roots. The colonization rates in the leaves were very low in all the three treatments with the highest being only 5\% from EABb04 inoculated treatment. 
Table 4 Colonization rates (\%) of $B$. bassiana root inoculated cabbage

\begin{tabular}{cccc}
\hline Strain & Leaf & Stem & Root \\
\hline Control & 0 & 0 & 0 \\
Bb03032 & 2.5 & 5.8 & 20.0 \\
EABb04 & 5.0 & 12.5 & 30.0 \\
Naturalis & 3.3 & 12.5 & 30.0 \\
\hline
\end{tabular}

Ten plants (=replicates) were used in each treatment. Three leaves per plant were obtained, four leaf discs were cut from each plant, twelve stem segments and three root segments per plant were used for colonization analysis.

In the feeding trials, the larvae feeding on $B$. bassiana treated leaves have a significant weight loss $(p<0.0001)$ compared to the non-inoculated control and also needed significantly longer to pupate $(p=0.012)$ and emerge $(p<0.01)$ (Figure 1$)$. DBM larvae feeding on control leaves needed $5.4 \pm 0.3$ days to pupate and $9.4 \pm 0.3$ days to emerge whilst larvae feeding on ATP02 inoculated leaves needed an average $6.8 \pm 0.5$ days to pupate and $11.3 \pm 0.6$ days to emerge; all larvae survived until pupation and only one larva from the ATP02 group did not successful emerge. However, Beauveria white mycelium did not grow out from the dead pupa which was kept on moisture paper in a sterile petri dish.

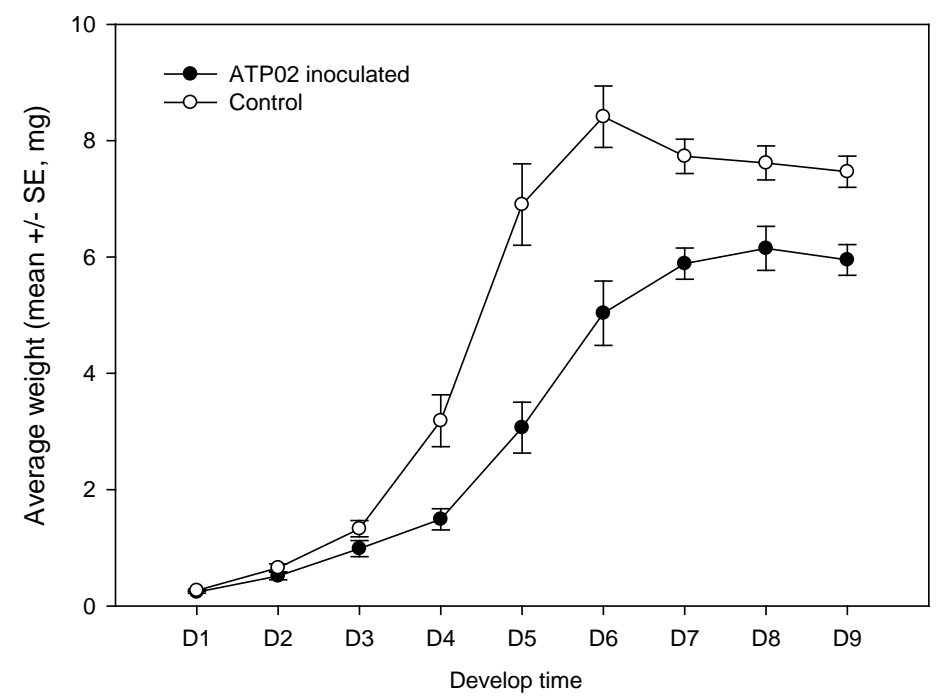

Figure 1 Weight of $P$. xylostella larvae after feeding on detached leaves from control and ATP 02 inoculated leaves (10 replicates/treatment; SE = standard error of the mean) 
In the oviposition bioassay, DBM adults significantly preferred the control plants over inoculated plants. In EABb04 inoculated trials, DBM laid an average $72.9 \pm 5.2$ eggs on control plants and $51.3 \pm 9.2$ eggs on EABb04 inoculated plants (goodness of fit for total number of eggs: $\mathrm{p}<0.001$ ); the same pattern was discovered from the Naturalis treatments as $69.9 \pm 8.1$ eggs were found on the control plants and $51.1 \pm 9.9$ eggs were found on treated plants (goodness of fit for total number of eggs: $p<0.001$ ).

\section{Discussions}

Inoculation of cabbage with $B$. bassiana did not show disease symptoms compared to non-inoculated controls. This indicates that B. bassiana colonization was not detrimental to the host plant. Furthermore B. bassiana was able to colonize cabbage plants with different inoculation methods.

A variety of major factors such as host species and cultivar, endophytes species and strain, concentration of the inoculum or age and growing conditions of the host plant can influence the establishment of the artificial introduction of entomopathogenic endophytes (Kessler et al. 2003, Tefera and Vidal 2009, Parsa et al. 2013). The level of B. bassiana colonization is substantially affected by inoculation method and plant growth medium (Tefera and Vidal (2009)): under non-sterile soil condition, either a leaf, seed and soil inoculation was performed, whereby a leaf spray inoculation resulted in the highest colonization rate in the leaf and stem of the host sorghum. A soil drench and seed inoculation resulted in low colonization efficiency. A similar pattern was found in the experiment conducted by Parsa et al. (2013), who compared a leaf spray and soil drench inoculation in common bean (Phaseolus vulgaris): the leaf spray method resulted in a higher colonization rate in the leaves and the soil inoculation resulted in a higher colonization rate in the root. In the here presented study, inoculation method and fungal strain both played an important role in the endophytic colonization of B. bassiana in cabbage tissue. The overall colonization rate in the leaf spray inoculation was higher than in the shoot and root inoculation. Moreover, different fungal strains performed differently in the three inoculation 
treatments. In the leaf and root inoculation treatments, Bb03032 had the lowest overall colonization rate, whereas the colonization of Naturalis was lowest in the shoot inoculated treatments; strain ATP01 and EABb04 showed a relatively higher colonization rates in all the trials.

B. bassiana can colonize the plants as an endophyte in host plants especially at the inoculated sites. Studies on the relationship between its distribution and the growth of host plant have been limited. Endophytic B. bassiana extensively colonizes parenchyma cells inter- and intracellularly; additionally it was also found in vascular (protoxylem) tissue (Gomez-Vidal et al. 2006). The inoculated fungi need time to spread from the inoculated site to the whole plant. As described by Posada and Vega (2005), inoculated B. bassiana exhibits a sharp increase in the colonization rate in cocoa seedlings from the first check period (one month after inoculation) to the second check period (2 month after inoculation). B. bassiana can also successfully establish in jute with an increased colonization rate from day 30 to 60 after inoculation (Biswas et al. 2012). However, some studies showed that the fungal colonization rates can decrease within certain periods. Study from Akello et al. (2007a) illustrated that, the percentage of $B$. bassiana colonization gradually declines over the 4 month assessment period with a root inoculation. A similar result was also found in B. bassiana colonized young date palm stem tissue (Gomez-Vidal et al. 2006). In this study the same leaf which was checked at 6-day intervals and also showed higher colonization rates during the first sampling dates. Although not proven yet, the decline in the percentage colonization might either be due to the competition in the host or the faster growth of the plants, outgrowing the expanding of the endophytic fungi.

All tested strains had the highest colonization in the inoculated leaves with a leaf inoculation; however, newly emerged leaves always had lower colonization rates with ongoing host plant growth; in the root inoculation treatments, a similar pattern was found as the inoculated root system had the highest colonization rate. In summary $B$. bassiana could colonize all plant tissues, but the introduced entomopathogenic fungus 
was only well established in the inoculated site of the associated inoculation method but poorly spread into the other plant tissue. This suggests that B. bassiana is a poor competitor and grows was very slowly in the host plants. The endophyte might establishe in the whole plant over a longer period than tested in this study.

The ultimate goal for our study is to develop an efficient inoculation method to introduce the $B$. bassiana into the host plant, which would be able to provide durable systemic resistance against herbivory and/or disease. The colonization of the enodphytic B. bassiana significantly affected the development of the DBM larvae and the ovipositing choice of the adults in the bioassay; the mechanisms behind these findings are not well known. This study provides evidence that B. bassiana is able to colonize the cabbages as an endophyte and could also serve as a potential bio-control agent against DBM. Further work need to be done to optimize the inoculation process in order to get a better fungal endophytic establishment and to explore the mechanism on how the endophyte influences herbivory and oviposition preference by DBM.

\section{Acknowledgement}

We would like to thank Chinese Scholar Council (CSC) for the financial support.

\section{References}

Akello, J., T. Dubois, D. Coyne, and S. Kyamanywa. 2008. Endophytic Beauveria bassiana in banana (Musa spp.) reduces banana weevil (Cosmopolites sordidus) fitness and damage. Crop Prot 27: 1437-1441.

Akello, J., T. Dubois, D. Coyne, and S. Kyamanywa. 2009. The effects of Beauveria bassiana dose and exposure duration on colonization and growth of tissue cultured banana Musa sp.) plants. Biological control 49: 6-10.

Akello, J., T. Dubois, D. Coyne, C. Gold, and S. Kyamanywa. Year. Published. Colonization and persistance of the entomopathogenic fungus, Beauveria 
bassiana, in tissue culture of banana, pp. 857-861. In, African Crop Science Conference Proceedings, 2007a.

Akello, J., T. Dubois, C. S. Gold, D. Coyne, J. Nakavuma, and P. Paparu. 2007b. Beauveria bassiana (Balsamo) Vuillemin as an endophyte in tissue culture banana (Musa spp.). Journal of Invertebrate Pathology 96: 34-42.

Bing, L. A., and L. C. Lewis. 1991. Suppression of Ostrinia nubilalis (Hubner) (Lepidoptera, Pyralidae) by endophytic Beauveria bassiana (Balsamo) Vuillemin. Environ Entomol 20: 1207-1211.

Biswas, C., P. Dey, S. Satpathy, and P. Satya. 2012. Establishment of the fungal entomopathogen Beauveria bassiana as a season long endophyte in jute (Corchorus olitorius) and its rapid detection using SCAR marker. Biocontrol 57: 565-571.

Brownbridge, M., S. D. Reay, T. L. Nelson, and T. R. Glare. 2012. Persistence of Beauveria bassiana (Ascomycota: Hypocreales) as an endophyte following inoculation of radiata pine seed and seedlings. Biological control 61: 194-200.

Chase, A., L. Osborne, and V. Ferguson. 1986. Selective isolation of the entomopathogenic fungi Beauveria bassiana and Metarhizium anisopliae from an artificial potting medim. Florida Entomologist 69: 285-292.

Cherry, A., A. Banito, D. Djegui, and C. Lomer. 2004. Suppression of the stem-borer Sesamia calamistis (Lepidoptera; Noctuidae) in maize following seed dressing, topical application and stem injection with African isolates of Beauveria bassiana. Int J Pest Manage 50: 67-73.

Gomez-Vidal, S., L. V. Lopez-Llorca, H. B. Jansson, and J. Salinas. 2006. Endophytic colonization of date palm (Phoenix dactylifera L.) leaves by entomopathogenic fungi. Micron 37: 624-632.

Gurulingappa, P., G. A. Sword, G. Murdoch, and P. A. Mcgee. 2010. Colonization of crop plants by fungal entomopathogens and their effects on two insect pests when in planta. Biological Control 55: 34-41.

Humber, R. A. 1997. Fungi: Identification. Manual of techniques in insect pathology: 153. 
Jones, K. D. 1994. Aspects of the biology and biological control of the European corn borer in North Carolina. Ph.D. Dissertation, North Carolina State University.

Kessler, P., H. Matzke, and S. Keller. 2003. The effect of application time and soil factors on the occurrence of Beauveria brongniartii applied as a biological control agent in soil. Journal of Invertebrate Pathology 84: 15-23.

Parsa, S., V. Ortiz, and F. E. Vega. 2013. Establishing fungal entomopathogens as endophytes: towards endophytic biological control. Journal of visualized experiments: JoVE.

Petrini, O. 1991. Fungal endophytes of tree leaves, pp. 179-197, Microbial ecology of leaves. Springer.

Posada, F., and F. E. Vega. 2005. Establishment of the fungal entomopathogen Beauveria bassiana (Ascomycota: Hypocreales) as an endophyte in cocoa seedlings (Theobroma cacao). Mycologia 97: 1195-1200.

Posada, F., M. C. Aime, S. W. Peterson, S. A. Rehner, and F. E. Vega. 2007. Inoculation of coffee plants with the fungal entomopathogen Beauveria bassiana (Ascomycota : Hypocreales). Mycol Res 111: 748-757.

Posada, F. J., F. C. Chaves, T. J. Gianfagna, M. Pava-Ripoll, and P. Hebbar. 2010. Establishment of the fungal entomopathogen Beauveria bassiana as an endophyte in cocoa pods (Theobroma cacao L.). Revista UDCA actualidad \& divulgación científica 13: 71-78.

Quesada-Moraga, E., F. J. Munoz-Ledesma, and C. Santiago-Alvarez. 2009. Systemic protection of Papaver somniferum L. against Iraella luteipes (Hymenoptera: Cynipidae) by an endophytic strain of Beauveria bassiana (Ascomycota: Hypocreales). Environ Entomol 38: 723-730.

Reay, S. D., M. Brownbridge, B. Gicquel, N. J. Cummings, and T. L. Nelson. 2010. Isolation and characterization of endophytic Beauveria spp. (Ascomycota: Hypocreales) from Pinus radiata in New Zealand forests. Biological Control 54: 52-60.

Reddy, N. P., A. P. Ali Khan, U. K. Devi, H. C. Sharma, and A. Reineke. 2009. Treatment of millet crop plant ( Sorghum bicolor) with the entomopathogenic 
fungus ( Beauveria bassiana) to combat infestation by the stem borer, Chilo partellus Swinhoe (Lepidoptera: Pyralidae). Journal of Asia-Pacific Entomology 12: 221-226.

Tefera, T., and S. Vidal. 2009. Effect of inoculation method and plant growth medium on endophytic colonization of sorghum by the entomopathogenic fungus Beauveria bassiana. Biocontrol 54: 663-669.

Vega, F. E., F. Posada, M. C. Aime, M. Pava-Ripoll, F. Infante, and S. A. Rehner. 2008. Entomopathogenic fungal endophytes. Biological Control 46: 72-82.

Wilson, D. 1995. Endophyte - the evolution of a term, and clarification of its use and definition. Oikos 73: 274-276. 


\title{
Chapter 2
}

\section{Endophytic establishment of Beauveria bassiana and Trichoderma} harzianum in cabbage

Leilei Zhang and Stefan Vidal

\author{
Georg-August-University Goettingen \\ Department of Crop Sciences \\ Section of Agricultural Entomology \\ Grisebachstrasse 6 \\ 37077 Goettingen Germany
}




\begin{abstract}
Experiments were conducted to introduce Beauveria bassinana (strains: EABb04 and Naturalis) and Trichoderma harzianum (strains: T39 and Tu) as endophytes in cabbage (Brassica oleracea var. capitata). Two separate inoculations of $B$. bassiana and $T$. harzianum were done by injecting the spores into the stem of the same plant at 7 day intervals. Three weeks after the second inoculation, the fungal colonization of leaf, stem and root was assessed through selective medium plating and specific real time PCR.
\end{abstract}

Both $B$. bassiana and $T$. harzianum had a poor establishment in leaves: a very low recovery rate and inoculum DNA concentration were found in B. bassiana treatments while none of the two T. harzianum strains was found to colonize the host cabbage leaves.

Inoculated fungi were recovered from all the stem samples obtained at the inoculated sites. However, $3 \mathrm{~cm}$ above the inoculated sites, fewer than half of the ten plants were confirmed to be colonized by B. bassiana; meanwhile with one week less growth time, $T$. harzianum could colonize the stems better than B. bassiana as at least six out of ten stem segments were positive on the selective medium. The q-PCR results showed that the highest fungal DNA concentration was found in the stem; $B$. bassiana single inoculated treatments had higher B. bassiana DNA concentration in stems compared with the dual-inoculated treatments. Although T. harzianum had higher positive rates, the concentration in stems were very low; no significant differences were found from the T. harzianum single inoculated treatments and dual inoculated treatments.

Fungi $T$. harzianum can colonize the root better than $B$. bassiana. While $B$. bassiana was poorly established in the root, nearly all the root samples were found to be positive for $T$. harzianum in selective medium re-isolation and q-PCR although the fungal concentrations were very low. 
Oviposition choice experiment showed that the diamondback moth adults significantly preferred control plants to EABb04 inoculated plants, however, no significant preference were found between the T39 inoculated and control plants.

Keywords: Beauveria bassiana; endophyte; Plutella xylostella; Trichoderma harzianum; stem inoculation, quantitative PCR 


\section{Introduction}

The use of endophytic fungi as bio-control agents has been widely studied in recent years (Vega et al. 2008, Biswas et al. 2012). Most bio-control measures of these fungi are based on exogenous application which is very costly as the presence of unsuitable conditions may kill most of the conidia resulting in multiple applications. With an endophytic establishment, the beneficial fungi can be present continuously in the host plant which is more economic and ecological friendly. Beauveria bassiana (Balsamo) Vuillemin (Ascomycota: Hypocreales) and Trichderma harzianum Rifai (Ascomycota: Hypocreales) are important environmentally friendly bio-control agents and their potential to colonize the plants has being widely recognized.

Beauveria bassiana is the most widely studied entomopathogenic fungus which has been proved for its ability to colonize either naturally or in response to artificial inoculation in maize (Bing and Lewis 1991), potato (Jones 1994), cocoa (Posada and Vega 2005), coffee (Posada et al. 2007), banana (Akello et al. 2007), date palm (Gomez-Vidal et al. 2006), and sorghum (Tefera and Vidal 2009), opium poppy (Quesada-Moraga et al. 2009), cotton, pumpkin, wheat (Gurulingappa et al. 2010), Pinus radiate (Reay et al. 2010), jute (Biswas et al. 2012) and common bean (Parsa et al. 2013). Endophytic B. bassiana has been demonstrated to suppress the pests in many studies. Bing and Lewis (1991) reported that under suitable environmental conditions tunneling by European corn borer larvae Ostrinia nubilalis could be significantly reduced by endophytic B. bassiana in maize; Cherry et al. (2004) reported that artificially introduced $B$. bassiana in maize successfully suppresses the stem-borer Sesamia calamistis; furthermore Akello et al (2008) found out that endophytic B. bassiana could reduce the population of banana weevil Cosmopolites sordidus and Vega et al. (2008) proved that endophytic B. bassiana can be used in controlling coffee berry borer; most recently Reddy et al (2009) reported the use of endophytic B. bassiana to control the stem borer Chilo partellus.

For the well-known bio-control fungus T. harzianum, most scientists have been 
focusing on its establishment in the plant rhizosphere, which results in stimulating plant growth and eliciting plant defense reactions against pathogens (Howell 2003, Benitez et al. 2004, Harman 2006). However, besides working as bio-control agent to control plant pathogens, some strains of Trichoderma spp. are also able to work against insects (Ownley et al. 2010). Kaaya and Okech (1990) isolated Trichoderma spp. from field-collected Glossina pallidipes, which was found to be mildly pathogenic for adult G. pallidipes; in a study from Santamarina et al. (2002) $T$. harzianum extracts were found to lead to a high mortality in Oncopeltus fasciatus; Cardoza et al. (2006) reported T. harzanium could significantly reduce spruce beetle survival and reproduction; Shakeri and Foster (2007) reported that two strains (101645 and 206040) of T. harzianum induced a higher mortality in Tenebrio molitor larvae; Ganassi et al. (2009) reported that strain ITEM908 showed entomopathogenic activity which resulted in a significant decrease of aphid (Myzus persicae) survival. As T. harzianum is also widely recognized as an endophyte (Harman et al. 2004), therefore, it's very interesting to know the role of endophytic Trichoderma spp. in host plants against herbivores.

In this study, the diamondback moth (DBM), Plutella xylostella (Linnaeus) (Lepidoptera: Plutellidae) was used in the bioassay set ups. As a worldwide pest, DBM is an oligophagous species that feeds on economically important plants in Brassicaceae (Thorsteinson 1953). Following the rapid increase of Brassica vegetable production in the past decade (Zalucki et al. 2012), new challenges have to be faced against the management of DBM. Up to now, no biological control methods have proven to be effective enough, and the control of the pest heavily relied on insecticides, which resulted in its resistance to all major classes of insecticides. Up to 2014, according to Arthropod Pesticide Resistance Database, 92 compounds have been reported as resistant in DBM. Due to agricultural intensification and greater production of Brassicaceae crops, it was estimated that DBM could cause a great economic loss of US\$4-5 billion annually worldwide (Zalucki et al. 2012). To control the pest, synthetic chemical insecticides were heavily used in the past decades; 
however, the short life cycle and high reproduction level resulted in its fast developing of field resistance to all major classes of insecticides (Talekar and Shelton 1993, Furlong et al. 2013). Efficient bio-control methods are highly required to control this pest.

Currently, few studies have focused on the establishment of B. bassiana and T. harzianum as endophytes in cabbage plants. The interaction of the two fungi was also not studied. In this study, we introduced B. bassiana and T. harzianum as endophytes in the host plant cabbage. The persistence of the fungi in different parts of the host plant was monitored through cultivation and quantitative PCR techniques; meanwhile, oviposition bioassays were also employed to test the influence of endophytic fungi on DBM. The aim of the study is the evaluation of endophytic biological control.

\section{Materials and methods}

\subsection{Materials}

\subsubsection{Fungus:}

Table 1 Beauveria bassiana and Trichoderma harzianum strains used for endophytic establishment in cabbage

\begin{tabular}{|c|c|c|c|}
\hline species & strains & Geographic origin & \\
\hline \multirow{4}{*}{ B. bassiana } & $\begin{array}{l}\text { EABb04/01-Tip } \\
\text { (EABb04) }\end{array}$ & Spain & $\begin{array}{lll}\text { stem-borer } & \text { Timaspis } & \text { papaveris } \\
\text { (Kieffer) larva } & & \end{array}$ \\
\hline & \multirow{3}{*}{ Naturalis ${ }^{\circledR}$} & \multirow{3}{*}{ USA } & ATCC74040-based \\
\hline & & & bioinsecticide) cotton boll weevil \\
\hline & & & Anthonomus grandis (Boheman) \\
\hline \multirow[t]{3}{*}{ T. harzianum } & $\mathrm{Tu}$ & Thailand & Uniseeds Co. Ltd. \\
\hline & \multirow{2}{*}{ T39 } & \multirow{2}{*}{ Israel } & (Makhteshim-Agan \\
\hline & & & Ltd., Tel Aviv, Israel) \\
\hline
\end{tabular}

Two strains of each fungal species were used in this experiment: B. bassiana (EABb04 (full name: EABb04/01-Tip) and Naturalis); T. harzianum (T39 and Tu). The origins and basic information of the fungal strains are listed in Table 1. Fungal 
conidia were harvested from 3-week-old PDA (potato dextrose agar) cultures. Conidial suspension was prepared in sterile $0.1 \%$ Tween 20 solution and the conidia concentration was adjusted to $1 \times 10^{8}$ conidia $\mathrm{ml}^{-1}$ using an improved Neubauer hemocytometer. The germination of all the fungal strains were checked on PDA medium using the method according to Posada and Vega (2005) .

\subsubsection{Host plant}

Cabbage plants, Brassica oleracea var. capitata L. (cultivar: Furchenkohl; Norddeutsche Pflanzenzucht Hans-Georg Lembke KG) were grown from seeds in seedling beds in the greenhouse chamber $\left(24 \pm 3^{\circ} \mathrm{C}\right.$; $80 \%$ relative humidity; 16L: 8D photoperiod). 10-day-old seedlings were transplanted into $11 \mathrm{~cm}$ diameter plastic pots with on-sterile soil (Fruhstorfer Erde Typ 25, Hawita Gruppe GmbH, peat fine structure with volcanic clay, pH: 5.7-6.3, Fertilization of 200-300mg N) and sand mixture (3:1 volume). Plants were grown in the chamber, irrigated regularly and fertilized once a week until needed (fertilizer: Hakaphos 2g/L (15\% N, 11\% $\mathrm{P}_{2} \mathrm{O}_{5}, 15 \%$ $\mathrm{K}_{2} \mathrm{O}, 1 \% \mathrm{Mg}, 0.1 \% \mathrm{Fe}, 0.1 \% \mathrm{Mn}, 0.04 \% \mathrm{Cu}, 0.025 \% \mathrm{~B}$ and $\left.0.005 \% \mathrm{Mo}\right)$ ).

\subsubsection{Insect}

DBM were obtained from the laboratory culture which was maintained in a cage $(90 \mathrm{~cm} \times 50 \mathrm{~cm} \times 50 \mathrm{~cm})$ in a climate controlled insectary $\left(18 \pm 2^{\circ} \mathrm{C}, 70 \pm 5 \%\right.$ relative humidity; L16:D8 photoperiod), fed with cabbage (cultivar: Furchenkohl) plants until needed.

\subsection{Inoculation}

4-week-old cabbage plants (about 6-leaf stage) were used for stem inoculation. A sterile needle was used to punch a hole on the stem $1.5 \mathrm{~cm}$ above the soil surface, following by pipetting $10 \mu \mathrm{l}$ B. bassiana spore suspension of strain EABb04 or Naturalis $\left(5 \times 10^{7}\right.$ conidia/ml) in the hole, after the suspension drops were fully 
absorbed (about 15min), a parafilm stripe was used to cover the inoculation area to avoid contamination and maintain a high humidity environment for spores germination. Beside the two treatments with B. bassiana strain EABb04 and Naturalis, another treatment sets was done using sterile $0.1 \%$ Tween 20 solution as control (Table 2).

Table 2 Stem inoculation design

\begin{tabular}{|c|c|c|c|c|c|}
\hline \multirow{2}{*}{\multicolumn{3}{|c|}{ Total: 9 treatments }} & \multicolumn{3}{|c|}{ First inoculation } \\
\hline & & & \multirow{2}{*}{$\begin{array}{l}\text { Sterile Tween } \\
20 \% \text { solution }\end{array}$} & \multicolumn{2}{|c|}{ B. bassiana } \\
\hline & & & & EABb04 & Naturalis \\
\hline \multirow{3}{*}{$\begin{array}{l}\text { Second } \\
\text { inoculation }\end{array}$} & \multicolumn{2}{|c|}{$\begin{array}{l}\text { Sterile Tween } 20 \% \\
\text { solution }\end{array}$} & $\begin{array}{l}\text { Serile-Sterile } \\
\text { (Control) }\end{array}$ & $\begin{array}{l}\text { EABb04-Sterile } \\
\text { (EABb04) }\end{array}$ & $\begin{array}{l}\text { Naturalis-Sterile } \\
\text { (Naturalis) }\end{array}$ \\
\hline & \multirow{2}{*}{ T. harzianum } & Т39 & $\begin{array}{l}\text { Sterile -T39 } \\
\text { (T39) }\end{array}$ & EABb04-T39 & Naturalis-T39 \\
\hline & & $\mathrm{Tu}$ & $\begin{array}{l}\text { Sterile-Tu } \\
\text { (Tu) }\end{array}$ & EABb04-Tu & Naturalis-Tu \\
\hline
\end{tabular}

There were ten replicates in each treatment

A second inoculation was done seven days after B.bassiana inoculation: $T$. harzianum (strain T39 or Tu) (5 $\times 10^{7}$ conidia/ml; 2nd treatment) or sterile $0.1 \%$ Tween 20 were inoculated $1 \mathrm{~cm}$ lower than the B.bassiana inoculation site using the same inoculation method (Table 2). Three weeks after the T. harzianum inoculation, fungal colonization rates were assessed in plant parts by placing them on selective medium as well as quantifying DNA with q-PCR.

\subsection{Selective medium re-isolation}

Beauveria selective medium (2\% oatmeal infusion, 2\% agar, 550 mg/L dodine, $0.25 \mathrm{~g} / \mathrm{L}$ chloramphenicol and $5 \mathrm{mg} / \mathrm{L}$ crystal violet; (Chase et al. 1986)) and Trichoderma selective medium (2\% agar, 2 g/L $\mathrm{MgSO}_{4}\left(7 \mathrm{H}_{2} \mathrm{O}\right), 0.9 \mathrm{~g} / \mathrm{L} \mathrm{K}_{2} \mathrm{HPO}_{4}, 0.15$ g/L KCl, 1.0 g/L N H $\mathrm{NO}_{3}$, 3.0 g/L glucose, 0.15 g/L rose bengal, 20 g/L agar, 0.25 g/L chloramphenicol, 0.2 g/L quintozene, 1.6 g/L metalaxyl (Elad and Chet 1983, Askew and Laing 1993)) were used to detect the presence of the fungi. 
Three weeks after the $T$. harzianum inoculation, stems $(5 \mathrm{~mm}$ segment at the inoculated site and $5 \mathrm{~mm}$ segment $3 \mathrm{~cm}$ above the inoculated site), roots and three leaves from different position (newly-emerged, medium and oldest) of each treated plants were collected and surface sterilized by the following sequence: $70 \%$ ethanol for one minute, $2 \% \mathrm{NaClO}$ for three minutes and rinsed three times in sterile water. The samples were dried under a clean bench. Four leaf discs per leaf were obtained using a sterile cork borer (diameter: $8 \mathrm{~mm}$ ), with 3 leaves per plant (newly-emerged, medium and oldest) and ten plants per treatment, 120 leaf discs per treatment were set up with a species specific selective medium. For stem and root samples, a $5 \mathrm{~mm}$ thick stem part was obtained $3 \mathrm{~cm}$ above the first inoculated site and a $2 \mathrm{~cm}$ long taproot part cut from the root system. Each sample was singly placed in a petri dish with selective medium. For stem samples, another $5 \mathrm{~mm}$ thick segment was obtained from the inoculated site and cultured in selective medium to confirm the inoculation was performed successfully. In order to exam whether the surface sterilization was successful, $100 \mu \mathrm{l}$ of the third rinsing water was pipetted and plated on selective medium. All the petri dishes were sealed with parafilm (Pechiney Plastic Packaging. Chicago Il) and incubated at $25^{\circ} \mathrm{C}$ for 3 weeks, by then the fungal existence was examined visually for fungal growth. Fungal growth was characterized as B. bassiana based on white dense mycelia, becoming cream to pale yellow at the edge (Humber 1997); T. harzianum based on the white, yellow, green mycelia and spores. The remains of the sterilized samples were well labeled and stored in $-80^{\circ} \mathrm{C}$ for the molecular check.

\subsection{Quantitative real-time PCR}

\subsubsection{Sample preparation}

Fungal DNA samples used for primer development and PCR assays were collected from one-week-old potato dextrose broth (PDB) culture. Mycelium was harvested by filtration. Surface sterilized plant samples and one-week-old fungal mycelium were freeze-dried and homogenized using the bead mill. 
Same CTAB DNA extraction method was used for all the fungal samples and plant samples. The steps were as follows. $50 \mathrm{mg}$ fine sample powders were transferred into a $2 \mathrm{ml}$ Eppendorf-tubes. 1ml CTAB buffer (supplemented with $2 \mu \mathrm{l}$ mercaptoethanol and $1 \mu \mathrm{l}$ proteinase; recipe for 1 liter: 7.4448 g Na-EDTA; 23 g Sorbitol; 10 g N-Laurylsarcosine, 8 g CTAB, 47 g Sodium Chloride, 10 g Polyvinylpyrrolidon 25.000-40.000, 10 mL 1 M Tris stock solution. $800 \mathrm{~mL}$ water) was added to each cup, mixed well; subsequently, the mixture was incubated at $42{ }^{\circ} \mathrm{C}$ for 10 minutes, then $65{ }^{\circ} \mathrm{C}$ for 10 minutes. In the following step, $800 \mu \mathrm{l}$ chlorofom/isoamylalcohol (24:1) was added, mixed and incubated on ice for $10 \mathrm{~min}$. After spinning at 8000 rpm for 10 minutes, the DNA was precipitated by transferring the supernatant into a new tube with $100 \mu \mathrm{l} 5 \mathrm{M} \mathrm{NaCl}$ and $200 \mu \mathrm{l} 30 \% \mathrm{PEG}$, incubating at room temperature for 5 minutes, then spinning at $14000 \mathrm{rpm}$ for 15 minutes. The supernatant was decanted and the pellet was then rinsed 2 times with $500 \mu \mathrm{l}$ 75\% ethanol, dried in SpeedVac and dissolved in $100 \mu \mathrm{l} 1 \times \mathrm{TE}$ buffer.

\subsubsection{Preparation of standards for quantitative real-time PCR}

The fungal genomic DNA was purified with phenol, and then the quality of the pure fungal DNA was checked by electrophoresis in $0.8 \%$ agarose gels. In order to determine the quantity of the DNA, densitometry values were compared with standard lambda phage DNA (methylated, from Escherichia coli host strain W3110) using Multi Analyst-Software (BioRad, Hercules, CA, USA) (Brandfass and Karlovsky 2008).

A dilution series (0.5 pg, 1 pg, 2.5 pg, 5 pg, 10 pg, 20 pg, 50 pg, 100 pg) of fungal genomic DNA was prepared to set up a standard curve in each PCR set. In addition to the standard curve three negative controls without template DNA (water instead) were also included in each PCR set. The plant samples were diluted 10 times and a PCR set was run to eliminate the matrix effect from the plant DNA which was made by comparing the q-PCR result from certain amount of fungal genomic DNA and fungal genomic DNA plus 10 times diluted cabbage DNA. 


\subsection{3 qPCR assays}

\section{B. bassiana:}

Two pairs of Beauveria primers were designed base on the 1765 bp Beauveria bassiana cuticle-degrading protease bassiasin I (bsn1) gene (GenBank Accession No. AF154118.1. Specificity tests were run for both of the primer pairs against the common fungi: Aspergillus niger, Beauveria bassiana (Bb3032, Canada1, EABb04, Naturalis, Columbia), Fusarium avenaceum, Fusarium equiseti, Fusarium proliferatum, Gliocladium Roseum, Metarhizium anisopliae, Microdochium nivale, Septoria nodorum, Trichoderma harzianum and Verticillium longisporum.

\section{bsn1-1}

Forward: 5’ -3’ CTGATGTAACTGCCTGTTCTCCTC

Reverse: 5’ -3’GATGCCTCAATTAGTGGCTTTCTC

Product size: 317 bp

\section{bsn1-2}

Forward: 5’ -3’ GCGTCAAGGTGCTCGAAGACAG

Reverse: 5’ -3’ TCTGGGCGGCATCCCTATTGT

Product size: 231bp

The iCycler System (CFX384 Real time system, Bio-Rad, Hercules, CA, USA) was used for the amplification and quantification of endophytic fungi. The reaction mixtures contained a final concentration of $1 \mu \mathrm{l} 10 \times$ Buffer (Bioline, Luckenwalde, Germany), $0.38 \mu \mathrm{l}$ of $50 \mathrm{mM} \mathrm{MgCl}_{2}$ (Bioline, Luckenwalde, Germany), $0.8 \mu \mathrm{l}$ of 2.5 mM dNTP (Bioline, Luckenwalde, Germany), $0.3 \mu$ of $10 \mu \mathrm{M}$ each primers (Invitrogen, Karlsruhe, Germany), $0.1 \mu \mathrm{l}$ of SybrGreen Mol Probes 1:1000 (Invitrogen, Karlsruhe, Germany), $0.02 \mu \mathrm{l}$ of DNA Polymerase (BIOTaq, Bioline, Luckenwalde, Germany), $6.1 \mu \mathrm{l}$ of sterile water and $1 \mu \mathrm{l}$ of sample DNA. The thermal cycling 
conditions for amplification were set as: an initial denaturation steps for 5 minutes at $94^{\circ} \mathrm{C}$, followed by 35 cycles each consisting of a denaturation step for 30 seconds at $94^{\circ} \mathrm{C}$, annealing for 20 seconds at $60{ }^{\circ} \mathrm{C}$, extension for 30 seconds at $72{ }^{\circ} \mathrm{C}$ and a plate read at the end of each cycle. This was followed by a final melting curve from $55^{\circ} \mathrm{C}$ to $95^{\circ} \mathrm{C}$ in $0.5^{\circ} \mathrm{C}$ increments held for 5 seconds at each temperature and a plate read at each temperature, which was used to determine the purity of the reaction products.

\section{T. harzianum}

T. harzianum specific primers (forward: 5'-TACAACTCCCAAACCCAATGTGA-3', reverse: 5'-CCGTTGTTGAAAGTTTTGATTCATTT-3') designed by Lopez-Mondejar et al. (2010) were used for the T. harzianum quantification. qPCR amplifications were performed in a total volume of $10 \mu \mathrm{l}$. The reaction mixtures contained a final concentration of $1 \mu \mathrm{l} 10 \times$ Buffer (Bioline, Luckenwalde, Germany), $0.6 \mu \mathrm{l}$ of $50 \mathrm{mM}$ $\mathrm{MgCl}_{2}$ (Bioline, Luckenwalde, Germany), $0.8 \mu \mathrm{l}$ of $2.5 \mathrm{mM} \mathrm{dNTP}$ (Bioline, Luckenwalde, Germany), $0.3 \mu \mathrm{l}$ of $10 \mu \mathrm{M}$ each primers (Invitrogen, Karlsruhe, Germany), $0.1 \mu \mathrm{l}$ of SybrGreen Mol Probes 1:1000 (Invitrogen, Karlsruhe, Germany), $0.02 \mu \mathrm{l}$ of DNA Polymerase (BIOTaq, Bioline, Luckenwalde, Germany), $5.88 \mu \mathrm{l}$ of sterile water and $1 \mu \mathrm{l}$ of sample DNA. The same thermal cycling conditions as Lopez-Mondejar et al. were used for amplification.

\section{Quantification}

Both of the Beauveria primer pairs can be used in DNA quantification. $10 \mathrm{pg}$ EABb04 gDNA was used to test the sensitivity of the two primer pairs; the results showed that Bsn1-2 (24.32) has a lower CT value than Bsn1-1(24.95), which indicated primer pair Bsn1-2 was more sensitive and would be used in the further $B$. bassiana quantification.

In all the PCR assays, two replicates for each DNA sample were assessed in PCR 
assay, the average data was used in the final data analysis. Since the amount of plant material and total volume of DNA volume was already known, the fungal DNA concentration in plant samples can be assessed from the PCR standard curve in each reaction.

\subsection{Oviposition choice assay}

The EABb04-Control (EABb04) inoculated plants and Control-T39 (T39) inoculated cabbage plants were used in the oviposition choice bioassay two weeks after the second inoculation. Insect rearing cages (size: $90 \mathrm{~cm} \times 50 \mathrm{~cm} \times 50 \mathrm{~cm}$ ) were used in the oviposition bioassay. Fresh pupae from lab culture were carefully collected into a plastic box $(18 \mathrm{~cm} \times 15 \mathrm{~cm} \times 5 \mathrm{~cm})$. After 5 days, 5 pairs of newly emerged male and female DBMs were placed in a test tube $(1.7 \mathrm{~cm}$ diameter $\times 10.5 \mathrm{~cm}$ length) which was closed off with a lid. Two cabbage plants were placed into each cage on opposite sides. One of the plant was fungal stem-inoculation-treated, while the other plant was control (treated with sterile water). The lid of the test tube with the DBM was removed and the test tube placed in the half way between the plants in the cage. To avoid the influence of the light on DBM behavior, the cages were left in darkness for $48 \mathrm{~h}$. To enhance the oviposition performance of DBM, parafilm stripes (Pechiney Plastic Packaging. Chicago Il) were used to cover the surface of the stems. The cabbage plants were then removed from the cage and the number of the eggs oviposited on each plant (including the pots, stems and leaves) was counted. $B$. bassiana strain EABb04 and T. harzianum strain T39 were tested for the influences of endophytic colonization on DBM oviposition behavior. Ten replicates (cages) for each treatment was applied in the assay.

\subsection{Statistical analysis}

Raw data were checked for normality and homogeneity of variance using the Shapiro-Wilk test. The variance within strains among different treatments for same plant tissue was analyzed with one way ANOVA and the overall egg laying number 
for each treatment was analyzed by goodness-of-fit test (SAS 9.3)

\section{Results}

\subsection{Selective medium re-isolation}

Spore germination rates of the B. bassiana and T. harzianum isolates used in the inoculation were over 90\%. No fungal growth was observed on the medium with $100 \mu$ l third time rinsing water.

Table 3 Colonization of cabbage leaves, stems and roots by Beauveria bassiana (strain EABb04 and Naturalis) and Trichoderma harzianum (strain T39 and Tu)

\begin{tabular}{ccccccc}
\hline \multirow{2}{*}{ Treatment } & \multicolumn{2}{c}{ Leaf } & \multicolumn{2}{c}{ Stem } & \multicolumn{2}{c}{ Root } \\
& B.bassiana & T.harzianum & B.bassiana & T.harzianum & B.bassiana & T.harzianum \\
\hline Control & 0 & 0 & 0 & 0 & 0 & 0 \\
Tu & - & 0 & - & 6 & - & 1 \\
T39 & - & 0 & - & 8 & - & 9 \\
Naturalis & 0 & - & 3 & - & 1 & - \\
Naturalis-Tu & 0 & 0 & 3 & 9 & 2 & 8 \\
Naturalis-T39 & 0 & 0 & 4 & 9 & 2 & 9 \\
EABb04 & 9 & - & 5 & - & 3 & - \\
EABb04-Tu & 9 & 0 & 4 & 8 & 2 & 7 \\
EABb04-T39 & 3 & 0 & 5 & 10 & 2 & 8 \\
\hline
\end{tabular}

Beauveria and Trichoderma selective medium was used to determine the recovery rate; stem samples were taken 3 cm above the inoculated sites; root samples were obtained from taproot; “-” represents no results; For leaf samples, a total number of 30 petri dish with120 leaf discs were collected for each treatment; for stem or root samples, a single sample was obtained from each plant.

Both B. bassiana and T. harzianum had a poor establishment in the leaves: a very low recovery rate were found in $B$. bassiana treatments while none of the two $T$. harzianum was found to colonize the host cabbage leaves (Table 3). All the inoculated site stem segments were confirmed with existence of the inoculated fungi. However, at $3 \mathrm{~cm}$ above the inoculated sites, fewer than half of the ten plants were confirmed to be colonized by B. bassiana; meanwhile despite with one week less growth time, $T$. harzianum could colonize the stems better as at least 6 out of 10 stem segments were positive on the selective medium (Table 3). In the root system, where B. bassiana was 
poorly established, all the root samples were found to be positive in selective medium re-isolation with T. harzianum (Table 3).

\subsection{Real time PCR quantification of fungal colonization}

The specificity of the self-designed $B$. bassiana primers against different fungal species was tested. The real time data as well as the products agarose gel showed that there was no specificity amplification for all of all the specificity test fungi except the B. bassiana strains. There was also no positive q-PCR signal from genomic DNA extracted from control cabbage, which indicates the primers don't bind to the cabbage genome and also eliminates the possibility of contamination in the inoculation section. The matrix effect was excluded by comparison of q-PCR results of pure fungal DNA and pure fungal DNA plus 10 times diluted control cabbage DNA.

\section{Quantification of endophytic B. bassiana}

Table 4 DNA concentration of $B$. bassiana (mean \pm SE) in stem inoculated cabbage by q-PCR (pg/mg plant material) using primer pair Reverse: 5'-3' TCTGGGCGGCATCCCTATTGT ; ;orward: 5' GCGTCAAGGTGCTCGAAGACAG

\begin{tabular}{ccccccc}
\hline \multirow{2}{*}{ Treatment } & \multicolumn{2}{c}{ Leaf } & \multicolumn{2}{c}{ Stem } & \multicolumn{2}{c}{ Root } \\
\cline { 2 - 7 } & mean \pm se & No. & mean \pm se & No. & mean \pm se & No. \\
\hline Control & nd & 0 & nd & 0 & nd & 0 \\
\hline Naturalis & $0.02 \pm 0.02 \mathrm{a}$ & 1 & $29.90 \pm 15.55 \mathrm{ab}$ & 5 & $1.67 \pm 1.13 \mathrm{ab}$ & 2 \\
Naturalis-Tu & $0.12 \pm 0.08 \mathrm{a}$ & 3 & $6.54 \pm 2.29 \mathrm{~b}$ & 10 & $4.45 \pm 0.10 \mathrm{ab}$ & 9 \\
Naturalis-T39 & $0.33 \pm 0.23 \mathrm{a}$ & 2 & $0.93 \pm 0.54 \mathrm{~b}$ & 3 & $0 \pm 0 \mathrm{~b}$ & 0 \\
EABb04 & $0.47 \pm 0.36 \mathrm{a}$ & 2 & $46.10 \pm 22.03 \mathrm{a}$ & 7 & $9.99 \pm 6.68 \mathrm{a}$ & 3 \\
EABb04 -Tu & $0.08 \pm 0.06 \mathrm{a}$ & 2 & $8.26 \pm 1.53 \mathrm{~b}$ & 10 & $4.27 \pm 2.02 \mathrm{ab}$ & 7 \\
EABb04 -T39 & $0.16 \pm 0.15 \mathrm{a}$ & 2 & $6.69 \pm 1.65 \mathrm{~b}$ & 9 & $1.04 \pm 0.45 \mathrm{ab}$ & 5 \\
\hline
\end{tabular}

Ten plants were used in q-PCR analysis, whereby 30 leaf sampls, 10 stem samples and 10 root samples were tested in q-PCR in each treatment; "No.” represents number of PCR positive results from the total number of tested samples; "nd" represents the fungal DNA concentration were under detectable amount; means ( \pm SE) followed by the same letter within a column are not significantly different at $P>0.05$.

As we can see from Table 4, the B. bassiana DNA content in the stem was higher than in the root, and only a very low positive rate and B. bassiana DNA concentration 
was discovered in the leaf samples. For 30 replicates per treatment in leaf samples, only up to three were giving a positive signal for q-PCR which indicated a very low colonization in the leaves and this was also confirmed by selective medium re-isolation. For stem samples, although not all the samples were positive for q-PCR (Table 5), B. bassiana single inoculated samples had higher average B. bassiana content than B. bassiana-T. harzianum double-inoculated treatments. When comparing the two B. bassiana strains, EABb04 was demonstrated to have both a higher average B. bassiana content and a higher q-PCR positive rate in nearly all the treatments (Table 4).

\section{Quantification of endophytic T. harzianum}

Table 5 Quantification of the concentration of T. harzianum in cabbage by q-PCR (Mean \pm SE pgDNA/mg plant material) using primer pair Reverse: 5'-CCGTTGTTGAAAGTTTTGATTCATTT-3' ; 5'-TACAACTCCCAAACCCAATGTGA-3 (Lopez-Mondejar et al., 2010)

\begin{tabular}{ccccccc}
\hline \multirow{2}{*}{ Treatments } & Leaf & \multicolumn{2}{c}{ Stem } & \multicolumn{2}{c}{ Root } \\
\cline { 2 - 7 } & mean \pm se & No. & mean \pm se & No. & mean \pm se & No. \\
\hline Control & nd & 0 & nd & 0 & nd & 0 \\
\hline Tu & nd & 0 & $0.11 \pm 0.04 \mathrm{a}$ & 9 & $0.05 \pm 0.01 \mathrm{~b}$ & 10 \\
T39 & nd & 0 & $0.38 \pm 0.19 \mathrm{a}$ & 9 & $0.14 \pm 0.03 \mathrm{~b}$ & 10 \\
Naturalis-Tu & nd & 0 & $0.12 \pm 0.03 \mathrm{a}$ & 9 & $0.14 \pm 0.08 \mathrm{~b}$ & 9 \\
Naturalis-T39 & nd & 0 & $0.13 \pm 0.05 \mathrm{a}$ & 7 & $0.15 \pm 0.04 \mathrm{ab}$ & 10 \\
EABb04 -Tu & nd & 0 & $0.04 \pm 0.01 \mathrm{a}$ & 8 & $0.31 \pm 0.08 \mathrm{a}$ & 8 \\
EABb04 -T39 & nd & 0 & $0.95 \pm 0.67 \mathrm{a}$ & 7 & $0.21 \pm 0.04 \mathrm{ab}$ & 10 \\
\hline
\end{tabular}

Ten plants were used in q-PCR analysis, whereby 30 leaf sampls, 10 stem samples and 10 root samples were tested in q-PCR; "No.” represents the number of PCR positive results from the total number of tested samples; "nd” represents the fungal DNA concentration were under detectable amount; means $( \pm \mathrm{SE}$ ) followed by the same letter within a column are not significantly different at $P>0.05$.

No positive sample was discovered from all the leaf samples while most of the samples from stem and root were positive (Table 5). Although the inoculation site was on the stem, in Naturalis-Tu, Naturalis-T39 and EABb04-Tu treatments, the $T$. harzianum DNA content in the root were even higher than in the stems from which we can draw the conclusion that $T$. harzianum can expand and colonize the root efficiently. No significant difference was found among the treatments within the same 
plant part of same T. harzianum strain (Table 5).

\section{Interaction of the two fungi species}

With a second inoculation of T. harzianum, although not always significant, both B. bassiana strains were discovered to have a lower amount of B. bassiana in stems; however, with the existence of $T$. harzianum, both stem and root samples were also found to have a higher PCR positive rate except in the Naturalis-T39 dual-inoculated treatment.

\subsection{Oviposition choice bioassay}

B. bassiana strain EABb04 and T. harzianum T39 were tested in the oviposition bioassay. The total number of eggs laid on control or inoculated plants within the same treatment was analyzed with Goodness-of-fit test. No significant difference ( $p=0.385$ ) was discovered for the ovipositive behavior between T39 treated plants (average $53.5 \pm 5.3$ ) and control (average $57.2 \pm 4.7)$. Significant difference $(p<0.001)$ was found between EABb04 (average $47.1 \pm 6.5$ ) inoculated and control plants (average $64.0 \pm 8.9$ ) which indicated DBM significantly preferred control plants over EABb04 inoculated plants.

\section{Discussions}

The recovery of the fungi in stem ( $3 \mathrm{~cm}$ above the inoculated site) and taproot indicated that the inoculated fungi were able to grow in both upward and downward from the inoculated site. As the sterilization might deactivate some of the endophytic fungi mycelium in the re-isolation, the molecular method was required to better demonstrate the distribution and quantity of the endophytic fungi in the host plant. As the distribution of the endophytic fungi in the plants is not homogenous, small amount of plant material (100 mg) were subsampled to extract DNA for PCR assays. Due to the large sampling error, the whole root, stem or leaf were used for subsamples in the 
experiment.

Entomopathogenic fungi are important regulators of insect populations with considerable potential as mycopesticides (Vega 2008, Parsa et al. 2013). Only in recent years, the fungal entomopathogens have been illustrated to occur as endophytes either naturally or in response to artificial inoculation. The existence of the entompathogenic fungi can promote the plant growth and reduce disease and herbivore resistance (Parsa et al. 2013). As one of the best known entomopathogen, $B$. bassiana has been well studied as a mycopesticide. In the past few years, its capability to act as an endophyte has also drawn wide attention from scientists. In this study, two B. bassiana strains were capable to colonize the cabbage plant, although the colonization rates were very low in some treatments. In the oviposition assay, with the establishment of $B$. bassiana strain EABb04, the DBM showed a significant preference for the control plant which proves the potential to use enophytic $B$. bassiana for biological control.

In this study, we found that although T. harzianum was inoculated one week afterwards, a higher colonization rate was found compared with B. bassiana which indicates that T. harzianum is more competitive in the plant. With a longer growing time, a higher T. harzianum content would be expected in the host plant. For the quantitative analysis, a higher $B$. bassiana DNA concentration and a lower recovery rate were found in $B$. bassiana single inoculated treatments. This might be due to the fact that the fungus was not competitive enough to expand in the host. In the dual-inoculated treatments, the establishment of the B. bassiana in the host plants was affected by T. harzianum, especially with the strain T39. With the existence of T39, the concentration of $B$. bassiana decreased rapdily which could be explained by the competition and mycoparasitism of T. harzianum. From q-PCR results, although with lower B. bassiana DNA concentration, more positive cases were found in most dual-inoculated treatments, unfortunately, further work needs to be done to explain this result. 
Currently, there is little doubt about the capacity of $T$. harzianum to act as a beneficial fungus to protect crops from phytopathogens, but there are different arguments about whether T. harzianum can act as an entomopathogen. Besides the evidence we listed in the introduction that T. harzianum has a positive effect on the herbivore control, studies also showed that the presence of some T. harzianum strains has no direct effect to the insect. For instance, Jayasimha and Henderson (Jayasimha and Henderson 2007) found the T. harzianum is not toxic to termites Coptotermes formosanus; studies on the pollinators also illustrate the health of both individual and the colony of honey bees and bumble bees was not be influenced by T. harzianum strain T22 and T39 (Brownold et al. 1997, Van der Steen et al. 2004, Mommaerts et al. 2008). Following this statement, some insects have been used as mechanical vectors to disperse T. harzianum. In 1995 (Vega et al. 1995), dusky sap beetle Carpophilus lugubris was reported to be used as a mechanical vector to disperse T. harzianum in the laboratory; Kovach et al. (Kovach et al. 2000) and Albano et al. (Albano et al. 2009) used honey bees Apis mellifera and bumble bees Bombus terrestris to spread $T$. harzianum to strawberries in order to control Botrytis spp.. All these studies showed T. harzianum has no direct influence on insects; however, with its well-known capability of producing secondary metabolites, enzymes and extracellular proteins, it has been proven that the Trichoderma genus is able to trigger the induced systemic resistance in many plants (Harman et al. 2004, Reino et al. 2008). Even though the Trichoderma induced systemic resistance has been well studied, limited research was focused on whether Trichoderma could have an indirect influence on insects. In 2013 (Battaglia et al. 2013), the influence of Trichoderma longibrachiatum colonization in tomato plants on the herbivore aphid Macrosiphum euphorbiae was studied. The result showed that the root colonization of $T$. longibrachiatum promotes tomato development and production of VOC, thus significantly improving the development and reproduction of $M$. euphorbiae and further affecting its natural enemies. In our study, T. harzianum was successfully established in the cabbage plants, but no significant difference was shown in the oviposition bioassay between the $T$. harzianum inoculated and control trials. This may be because either the endophytic 
expression of the T. harzianum did not affect the herbivore or the low content of fungi in the host was not sufficient to trigger the induced systemic resistance. Our data showed that as a very aggressive fungus, , T. harzianum was able to colonize the root effectively but with a small amount. However, the fungal colonization and fungal quantity of $T$. harzianum were assessed only 3 weeks after inoculation and with longer growth time, a higher colonization rate and fungal concentration can be expected. The high colonization rates in roots also indicated that $T$. harzianum can colonize the root system very effectively, as consequence root inoculation can be a potential good method to introduce T. harzianum as an endophyte.

\section{Acknowledgement}

We would like to thank Chinese Scholar Council (CSC) for the financial support and Prof. Dr. Petr Karlovsky for his suggestions and assistances concerning the primer design and real-time PCR.

\section{References}

Akello, J., T. Dubois, D. Coyne, and S. Kyamanywa. 2008. Endophytic Beauveria bassiana in banana (Musa spp.) reduces banana weevil (Cosmopolites sordidus) fitness and damage. Crop Prot 27: 1437-1441.

Akello, J., T. Dubois, C. S. Gold, D. Coyne, J. Nakavuma, and P. Paparu. 2007. Beauveria bassiana (Balsamo) Vuillemin as an endophyte in tissue culture banana (Musa spp.). Journal of Invertebrate Pathology 96: 34-42.

Albano, S., M. Chagon, D. de Oliveira, E. Houle, P. Thibodeau, and A. Mexia. 2009. Effectiveness of Apis mellifera and Bombus impatiens as dispensers of the Rootshield ${ }^{\circledR}$ biofungicide (Trichoderma harzianum, strain T-22) in a strawberry crop. Hell Plant Prot J 2: 57-66.

Askew, D. J., and M. D. Laing. 1993. An adapted selective medium for the 
quantitative isolation of Trichoderma species. Plant Pathol 42: 686-690.

Battaglia, D., S. Bossi, P. Cascone, M. C. Digilio, J. D. Prieto, P. Fanti, E. Guerrieri, L. Iodice, G. Lingua, M. Lorito, M. E. Maffei, N. Massa, M. Ruocco, R. Sasso, and V. Trotta. 2013. Tomato below ground-above fround interactions: Trichoderma longibrachiatum affects the performance of Macrosiphum euphorbiae and its natural antagonists. Mol Plant Microbe In 26: 1499-1499.

Benitez, T., A. M. Rincón, M. C. Limón, and A. C. Codón. 2004. Biocontrol mechanisms of Trichoderma strains. International microbiology 7: 249-260.

Bing, L. A., and L. C. Lewis. 1991. Suppression of Ostrinia nubilalis (Hubner) (Lepidoptera, Pyralidae) by endophytic Beauveria bassiana (Balsamo) Vuillemin. Environ Entomol 20: 1207-1211.

Biswas, C., P. Dey, S. Satpathy, and P. Satya. 2012. Establishment of the fungal entomopathogen Beauveria bassiana as a season long endophyte in jute (Corchorus olitorius) and its rapid detection using SCAR marker. Biocontrol 57: 565-571.

Brandfass, C., and P. Karlovsky. 2008. Upscaled CTAB-based DNA extraction and real-time PCR assays for Fusarium culmorum and F. graminearum DNA in plant material with reduced sampling error. Int J Mol Sci 9: 2306-2321.

Brownold, E., S. Flanders, and J. Kovach. 1997. The effect of Trichoderma harzianum on honey bee survival. NYS Fruit Project Reports Relating to IPM. Cornell Cooperative Extension, Cornell University, NYS IPM 214: 92-94.

Cardoza, Y. J., K. D. Klepzig, and K. F. Raffa. 2006. Bacteria in oral secretions of an endophytic insect inhibit antagonistic fungi. Ecol Entomol 31: 636-645.

Chase, A., L. Osborne, and V. Ferguson. 1986. Selective isolation of the entomopathogenic fungi Beauveria bassiana and Metarhizium anisopliae from an artificial potting medim. Florida Entomologist 69: 285-292.

Cherry, A., A. Banito, D. Djegui, and C. Lomer. 2004. Suppression of the stem-borer Sesamia calamistis (Lepidoptera; Noctuidae) in maize following seed dressing, topical application and stem injection with African isolates of 
Beauveria bassiana. Int J Pest Manage 50: 67-73.

Elad, Y., and I. Chet. 1983. Improved selective media for isolation of Trichoderma spp. or Fusarium spp. Phytoparasitica 11: 55-58.

Furlong, M. J., D. J. Wright, and L. M. Dosdall. 2013. Diamondback moth ecology and management: problems, progress, and prospects. Annu Rev Entomol 58: $517-+$

Ganassi, S., C. Altomare, and M. Sabatini. 2009. Interactions between fungi belonging to the genus Trichoderma and Myzus persicae (Hemiptera: Aphidoidea) to open new perspectives of biologicol control. Micologia Italiana 38: 3-9.

Gomez-Vidal, S., L. V. Lopez-Llorca, H. B. Jansson, and J. Salinas. 2006. Endophytic colonization of date palm (Phoenix dactylifera L.) leaves by entomopathogenic fungi. Micron 37: 624-632.

Gurulingappa, P., G. A. Sword, G. Murdoch, and P. A. Mcgee. 2010. Colonization of crop plants by fungal entomopathogens and their effects on two insect pests when in planta. Biological Control 55: 34-41.

Harman, G. E. 2006. Overview of mechanisms and uses of Trichoderma spp. Phytopathology 96: 190-194.

Harman, G. E., C. R. Howell, A. Viterbo, I. Chet, and M. Lorito. 2004. Trichoderma species - Opportunistic, avirulent plant symbionts. Nat Rev Microbiol 2: 43-56.

Howell, C. R. 2003. Mechanisms employed by Trichoderma species in the biological control of plant diseases: The history and evolution of current concepts. Plant Dis 87: 4-10.

Humber, R. A. 1997. Fungi: Identification. Manual of techniques in insect pathology: 153.

Jayasimha, P., and G. Henderson. 2007. Effect of Aspergillus flavus and Trichoderma harzianum on survival of Coptotermes formosanus (Isoptera : Rhinotermitidae). Sociobiology 49: 135-141.

Jones, K. D. 1994. Aspects of the biology and biological control of the European corn 
borer in North Carolina. Ph.D. Dissertation, North Carolina State University.

Kaaya, G. P., and M. A. Okech. 1990. Microorganisms associated with tsetse in nature -preliminary-results on isolation, identification and pathogenicity. Insect Sci Appl 11: 443-448.

Kovach, J., R. Petzoldt, and G. E. Harman. 2000. Use of honey bees and bumble bees to disseminate Trichoderma harzianum 1295-22 to strawberries for Botrytis control. Biological Control 18: 235-242.

Lopez-Mondejar, R., A. Anton, S. Raidl, M. Ros, and J. A. Pascual. 2010. Quantification of the biocontrol agent Trichoderma harzianum with real-time TaqMan PCR and its potential extrapolation to the hyphal biomass. Bioresource Technol 101: 2888-2891.

Mommaerts, V., G. Platteau, J. Boulet, G. Sterk, and G. Smagghe. 2008. Trichoderma-based biological control agents are compatible with the pollinator Bombus terrestris: A laboratory study. Biological Control 46: 463-466.

Ownley, B. H., K. D. Gwinn, and F. E. Vega. 2010. Endophytic fungal entomopathogens with activity against plant pathogens: ecology and evolution. Biocontrol 55: 113-128.

Parsa, S., V. Ortiz, and F. E. Vega. 2013. Establishing fungal entomopathogens as endophytes: towards endophytic biological control. Journal of visualized experiments: JoVE.

Posada, F., and F. E. Vega. 2005. Establishment of the fungal entomopathogen Beauveria bassiana (Ascomycota: Hypocreales) as an endophyte in cocoa seedlings (Theobroma cacao). Mycologia 97: 1195-1200.

Posada, F., M. C. Aime, S. W. Peterson, S. A. Rehner, and F. E. Vega. 2007. Inoculation of coffee plants with the fungal entomopathogen Beauveria bassiana (Ascomycota : Hypocreales). Mycol Res 111: 748-757.

Quesada-Moraga, E., F. J. Munoz-Ledesma, and C. Santiago-Alvarez. 2009. Systemic protection of Papaver somniferum L. against Iraella luteipes (Hymenoptera: Cynipidae) by an endophytic strain of Beauveria bassiana 
(Ascomycota: Hypocreales). Environ Entomol 38: 723-730.

Reay, S. D., M. Brownbridge, B. Gicquel, N. J. Cummings, and T. L. Nelson. 2010. Isolation and characterization of endophytic Beauveria spp. (Ascomycota: Hypocreales) from Pinus radiata in New Zealand forests. Biological Control 54: 52-60.

Reddy, N. P., A. P. Ali Khan, U. K. Devi, H. C. Sharma, and A. Reineke. 2009. Treatment of millet crop plant ( Sorghum bicolor) with the entomopathogenic fungus ( Beauveria bassiana) to combat infestation by the stem borer, Chilo partellus Swinhoe (Lepidoptera: Pyralidae). Journal of Asia-Pacific Entomology 12: 221-226.

Reino, J. L., R. F. Guerrero, R. Hernández-Galán, and I. G. Collado. 2008. Secondary metabolites from species of the biocontrol agent Trichoderma. Phytochemistry Reviews 7: 89-123.

Santamarina, M., J. Roselló, R. Llacer, and V. Sanchis. 2002. Antagonistic activity of Penicillium oxalicum Corrie and Thom, Penicillium decumbens Thom and Trichoderma harzianum Rifai isolates against fungi, bacteria and insects in vitro. Revista iberoamericana de micología 19: 99-103.

Shakeri, J., and H. A. Foster. 2007. Proteolytic activity and antibiotic production by Trichoderma harzianum in relation to pathogenicity to insects. Enzyme Microb Tech 40: 961-968.

Talekar, N. S., and A. M. Shelton. 1993. Biology, ecology, and management of the diamondback moth. Annual Review of Entomology 38: 275-301.

Tefera, T., and S. Vidal. 2009. Effect of inoculation method and plant growth medium on endophytic colonization of sorghum by the entomopathogenic fungus Beauveria bassiana. Biocontrol 54: 663-669.

Thorsteinson, A. 1953. The chemotactic responses that determine host specificity in an oligophagous insect (Plutella maculipennis (Curt.) Lepidoptera). Canadian Journal of Zoology 31: 52-72.

Van der Steen, J., C. Langerak, C. Van Tongeren, and A. Dik. Year. Published. Aspects of the use of honeybees and bumblebees as vector of antagonistic 
micro-organisms in plant disease control, pp. 41-46. In, Proceedings of the section experimental and applied entomology-Netherlands entomological society, 2004.

Vega, F. E. 2008. Insect pathology and fungal endophytes. Journal of invertebrate pathology 98: 277-279.

Vega, F. E., P. F. Dowd, and R. J. Bartelt. 1995. Dissemination of microbial agents using an autoinoculating device and several insect species as vectors. Biological Control 5: 545-552.

Vega, F. E., F. Posada, M. C. Aime, M. Pava-Ripoll, F. Infante, and S. A. Rehner. 2008. Entomopathogenic fungal endophytes. Biological Control 46: 72-82.

Zalucki, M. P., A. Shabbir, R. Silva, D. Adamson, S. S. Liu, and M. J. Furlong. 2012. Estimating the economic cost of one of the world's major insect pests, Plutella xylostella (Lepidoptera: Plutellidae): just how long is a piece of string? J Econ Entomol 105: 1115-1129. 


\section{Chapter 3}

\section{Trichoderma harzianum colonization triggers plant systemic defense}

\section{against herbivores}

Leilei Zhang, Sandra Aragón and Stefan Vidal

Georg-August-University Goettingen

Department of Crop Sciences

Section of Agricultural Entomology

Grisebachstrasse 6

37077 Goettingen Germany 
Abstract

Whether Trichoderma harzianum established in host plant as an endophyte affects the herbivore has been examined in this study. Two strains of bio-control fungus T. harzianum (T39 and Tu) were introduced to cabbage plant by root inoculation. The colonization status of T. harzianum in cabbage was checked by selective medium re-isolation and quantitative real-time PCR which illustrated that, both strains can colonize the root system of the host plant efficiently but hardly reached the leaves.

Three behavioral bioassays were performed using diamondback moth (DBM) Plutella xylostella. 1) The no-choice feeding experiment showed that although the DBM fed on the inoculated plants had slightly weight loss compared with control, no significant difference was found. 2) The dual-choice feeding assay showed that DBM larvae preferred to feed on control plants more than on inoculated plants. 3) The dual-choice oviposition assay also demonstrated that with when DBM adults were exposed to both treatments, the DBM laid significantly more eggs on control plants over T. harzianum inoculated plants.

Aboveground volatiles organic compounds (VOCs) from control and $T$. harzianum inoculated cabbages were collected with a modified "push-pull” headspace collection chamber. The trapped VOCs were analyzed by Gas Chromatography coupled to a Mass Spectrometer (GC-MS). Analysis showed that T. harzianum colonization conducted strong quantitative differences between inoculated and control plants. Although not always significant, inoculated plants exhibited a relative lower amount of 1-hexyl acetate, hexanal, (Z)-2-pentenyl acetate, (Z)-3-hexen-1-ol, (Z)-3-hexenyl acetate and a higher amount of 2-ethyl-1-hexanol and all terpenes (except no $\alpha$-farnesene in Tu treated plants was detected).

Key words: Trichoderma harzianum, volatiles, colonization, endophyte, systemic defense, volatile organic compound 


\section{Introduction}

Trichoderma spp. are free-living fungi that are common in soil and root ecosystems; their capacity to control plant pathogenic fungi has been widely studied (Harman et al. 2004). They are also widely recognized as endophytes which could live within host plant tissues without causing any visible symptoms of diseases (Wilson 1995). Direct and indirect mechanisms have been employed by Trichoderma spp. to attack target pathogenic fungi. Strategies include mycoparasitism, antibiosis, competition, and enzymes producing leading to a direct impact to the target fungi (Howell 2003). Besides direct strategies, Trichoderma spp. have been shown to indirectly influence the pathogenic fungi by inducing localized or systemic resistance systems in host plants (De Meyer et al. 1998).

Although plenty of studies have been done regarding to the interaction of Trichoderma spp. and pathogenic fungi, only limited researchers have focused on the relationship between Trichoderma spp. and insects. Until recently, there are still some different arguments about whether Trichoderma spp. could work against insects. Several studies revealed that besides controlling plant pathogenic fungi, Trichoderma spp. also have the ability to work against insects. (Ownley et al. 2010). Kaaya and Okech (1990) isolated Trichoderma spp. from field-collected Glossina pallidipes, and found it was mildly pathogenic for adult G. pallidipes; in a study from Santamarina et al. (2002) T. harzianum extracts was found leading to a high mortality in Oncopeltus fasciatus; Cardoza et al. (2006) reported T. harzanium significantly reduced spruce beetle survival and reproduction; Shakeri and Foster (2007) reported that two strains (101645 and 206040) of T. harzianum induced a higher mortality in Tenebrio molitor larvae; Ganassi et al. (2009) reported strain ITEM908 showed entomopathogenic activity resulting in a significant decrease of aphid (Myzus persicae) survival. However, some studies also showed that the presence of Trichoderma spp. has no direct effect on the insects. Studies found that T. harzianum is not toxic to termites Coptotermes formosanus (Jayasimha and Henderson 2007), and some pollinators such 
as honey bees Apis mellifera, bumble bees Bombus terrestris (Brownold et al. 1997, Van der Steen et al. 2004, Mommaerts et al. 2008). Honey bees, bumble bees and dusky sap beetle Carpophilus lugubris were even studied for their potential to act as vectors to disperse T. harzianum (Vega et al. 1995, Kovach et al. 2000, Albano et al. 2009). Currently, the effect of T. harzianum, the most ubiquitous biological control fungus, on plant defense induction against herbivores has not been studied in detail. Therefore, in this study, we did the feeding and oviposition bioassay to assess the influence of T. harzianum colonization on herbivore behavior.

Insect herbivores are known to use visual and chemical cues to seek for host plants or oviposition sites (Bernays and Chapman 1994, Renwick and Chew 1994), thus plant volatiles play an important role in the host-location process for herbivores insects and natural enemies (Bruce et al. 2005). The release of the volatiles organic compounds (VOCs) was associated with a range of biotic and abiotic stress factors (Holopainen and Gershenzon 2010). As a generalization, abiotic stress factors such as temperature (Tingey et al. 1980), high light intensity (Duhl et al. 2008), water stress (Ebel et al. 1995, Vallat et al. 2005) and salt stress (Loreto et al. 2002) have been reported to increase the VOCs emission which results in the increase of a variety of different terpene volatiles in a range of woody and herbaceous species. Biotic stress also has been proved to induce the emission of plant volatiles. Biotic factors including herbivore damage (Dudareva et al. 2006), mechanical damage (Fontana et al. 2009) and pathogenic attack (Leitner et al. 2008, Yi et al. 2009) have been proved to increase the release of inducible VOCs, especially green leaf volatiles (GLVs) and terpenes. However, there are only a few studies related to the interaction of endophytic fungi colonization and headspace volatile emission (Battaglia et al. 2013).

The diamondback moth (DBM) Plutella xylostella, which cause a great economic loss of US\$4-5 billion annually worldwide (Zalucki et al. 2012), is one of the most serious pests of cruciferous crops throughout the world (Talekar and Shelton 1993). Cruciferous plants emit a complex mixture of biogenic VOCs which are 
known to be important cues for host selection by cruciferous pests and their parasitoids, aiding both finding and recognition of the host plant (Bartlet et al. 1993, Alford et al. 2003). Electrophysiology response studies already proved that many VOCs including Z-3-hexenyl acetate, Z-3-hexen-1-ol, nonanol, E-myrcene, $\alpha$-pinene, D-limonene, $\alpha$-terpinene, $\beta$-caryophyllene and $\beta$-caryophyllene DBM were bioactive compounds for the DBM (Han et al. 2001). Behavior test also confirmed that the cabbage green leaf volatiles (GLVs) [(Z)-3-hexenyl acetate, (E)-2-hexenal, (Z)-3-hexen-1-ol, hexanal, 1-hexanol, 1-hexen-3-ol, and hexyl acetate] were able to induced attractant/arresting behavior in DBM in wind tunnel (Reddy and Guerrero 2000). As the volatiles are important cues for the DBM, in this study, we demonstrated that $T$. harzianum was able to colonize the cabbage plants endophytically and this colonization also affected the host plant volatile profiles and the DBM behavioral responses.

The objectives of this study were (i) to introduce T. harzianum as an endophyte in cabbage plant, (ii) to check the colonization efficiency of the endophytic $T$. harzianum, (iii) to evaluate the effect of T. harzianum colonization on the herbivore DBM, (iv) to examine the influence of T. harzianum colonization on volatile profiles.

\section{Materials and methods}

\subsection{Marterials}

\subsubsection{Fungus:}

Trichoderma harzianum strain T39 (Makhteshim-Agan LtD, Tel Aviv, Israel) and Tu (Uniseeds Co, Ltd, Thailand) were used in current study. To prepare the fungal suspension, fungal conidia were harvested from 3-week-old PDA (potato dextrose agar) cultures with a camel hairbrush into a sterile beaker which had $500 \mathrm{ml}$ sterile $0.1 \%$ Tween 20 solution. The suspensions were then homogenized by using a magnetic stirrer for 5 minutes and adjusted to $2 \times 10^{7}$ conidia/ml using an improved Neubauer 
hemocytometer. The germination rates were examined by PDA medium using the method according to Posada and Vega (2005).

\subsubsection{Host plant}

Cabbage plants, Brassica oleracea var. capitata L. (cultivar: Furchenkohl; Norddeutsche Pflanzenzucht Hans-Georg Lembke KG) were grown in seedling trays from seeds in a greenhouse chamber $\left(24 \pm 3^{\circ} \mathrm{C}\right.$; $80 \%$ relative humidity; 16L: 8D photoperiod). 10-day-old seedlings were transplanted into $11 \mathrm{~cm}$ diameter pots with non-sterile soil (Fruhstorfer Erde Typ 25, Hawita Gruppe GmbH, peat fine structure with volcanic clay, pH: 5.7-6.3, Fertilization of 200-300mg N) and sand mixture (3:1 volume). Plants were grown in the chamber, irrigated regularly and fertilized once a week until needed (fertilizer: Hakaphos $2 \mathrm{~g} / \mathrm{L}\left(15 \% \mathrm{~N}, 11 \% \mathrm{P}_{2} \mathrm{O}_{5}, 15 \% \mathrm{~K}_{2} \mathrm{O}, 1 \% \mathrm{Mg}\right.$, 0.1\% Fe, 0.1\% Mn, 0.04\% Cu, $0.025 \% \mathrm{~B}$ and $0.005 \% \mathrm{Mo})$ ).

\subsubsection{Insect}

The diamondback moths (DBM) used in the bioassays were collected from a laboratory culture which has been kept in a cage $(90 \mathrm{~cm} \times 50 \mathrm{~cm} \times 50 \mathrm{~cm})$ in a climate controlled insectary $\left(18 \pm 2^{\circ} \mathrm{C}, 70 \pm 5 \%\right.$ relative humidity; L16:D8 photoperiod), fed with cabbage plants (cultivar: Furchenkohl) until needed.

\subsection{Root inoculation}

Two weeks after the transplanting, the seedlings (growth stage: 4 leaves) were gently removed from the pots and the soil was washed off under the tap water. The inoculation was performed by immersing the root of the seedling into $2 \times$ $10^{7}$ conidia/ml spore suspension or sterile water (control treatment) for 30 minutes, then the seedlings were transplanted into a new pot and placed in the greenhouse chamber, irrigated regularly and fertilized once a week. Inoculated plants were kept in the chamber for another three weeks, by then they were used for volatile profiles extraction, fungal re-isolation, q-PCR detection, feeding experiments and oviposition 
choice assay.

\subsection{Selective medium re-isolation}

Trichoderma selective medium (2\% agar, $2 \mathrm{~g} / \mathrm{L} \mathrm{MgSO}_{4}\left(7 \mathrm{H}_{2} \mathrm{O}\right), 0.9$ g/L K $\mathrm{K}_{2} \mathrm{HPO}_{4}$, $0.15 \mathrm{~g} / \mathrm{L} \mathrm{KCl}, 1.0$ g/L N H $\mathrm{NO}_{3}, 3.0 \mathrm{~g} / \mathrm{L}$ glucose, $0.15 \mathrm{~g} / \mathrm{L}$ rose bengal, $20 \mathrm{~g} / \mathrm{L}$ agar, $0.25 \mathrm{~g} / \mathrm{L}$ chloramphenicol, $0.2 \mathrm{~g} / \mathrm{L}$ quintozene, $1.6 \mathrm{~g} / \mathrm{L}$ metalaxyl (Elad and Chet 1983, Askew and Laing 1993)) were used to detect the existence of the fungi.

Three weeks after the inoculation, stems (six $5 \mathrm{~mm}$ segments each plant), roots (three $2 \mathrm{~cm}$ taproot segments each plant) and three leaves from different position (newly emerged, medium and oldest) of each treated plants were collected and surface sterilized by immersing the samples in $70 \%$ ethanol for 1 minute, $2 \% \mathrm{NaClO}$ for 3 minutes, then rinsed in sterile water for three times. The samples were left in clean bench until the surface was dry. Four leaf discs per leaf were obtained using a sterile cork borer (diameter: $8 \mathrm{~mm}$ ), with three leaves per plant and 10 plants per treatment. A total number 120 leaf discs per treatment were collected and put on species specific selective medium. For stem and root samples, each segment was placed in a single selective medium petri dish. In order to examine whether the surface sterilization was successful, $100 \mu \mathrm{l}$ of the third time rinsing water was pipetted and plated on selective medium. All the petri dishes were sealed with parafilm (Pechiney Plastic Packaging. Chicago Il) and incubated in an incubator at $25^{\circ} \mathrm{C}$ for three weeks, by then the fungal existence was examined visually for $T$. harzianum fungal growth which was characterized based on the white, yellow, green mycelia and spores. After keeping the selective medium in incubator for three weeks, the colonization percentage was calculated by: \% colonization $=$ number of leaf discs showing $T$. harzianum outgrowth divided by the total number of incubated leaf discs $\times 100 \%$. The remaining of the sterilized samples were well labeled and stored in $-80^{\circ} \mathrm{C}$ for the molecular check.

\subsection{Quantitative real-time PCR}




\subsubsection{Sample preparation}

Fungal DNA samples used in PCR assays were obtained from one-week-old potato dextrose broth (PDB) culture. Mycelium was harvested by filtration. Plant samples were already prepared in the 2.3 which were ready for lyophilization directly. All the samples were freeze-dried and homogenized using the bead mill.

Same CTAB DNA extraction method was used for all pure fungal samples and plant samples. The steps were as follows. $50 \mathrm{mg}$ fine sample powders were transferred into a $2 \mathrm{ml}$ Eppendorf-tubes. $1 \mathrm{ml}$ CTAB buffer (supplemented with $2 \mu \mathrm{l}$ mercaptoethanol and $1 \mu \mathrm{l}$ proteinase; recipe for 1 liter: 7.4448 g Na-EDTA; 23 g Sorbitol; 10 g N-Laurylsarcosine, 8 g CTAB, 47 g Sodium Chloride, 10 g Polyvinylpyrrolidon 25.000-40.000, $10 \mathrm{~mL} 1 \mathrm{M}$ Tris stock solution. $800 \mathrm{~mL}$ water) was added to each cup, mixed well; subsequently, the mixture was incubated at $42{ }^{\circ} \mathrm{C}$ for 10 minutes, then $65^{\circ} \mathrm{C}$ for 10 minutes. In the following step, $800 \mu \mathrm{l}$ chlorofom/isoamylalcohol(24: 1) was added, mixed and incubated on ice for 10 minutes. After spinning at 8000 rpm for 10 minutes, the DNA was precipitated by transferring the supernatant into a new tube with $100 \mu \mathrm{l} 5 \mathrm{M} \mathrm{NaCl}$ and $200 \mu \mathrm{l} 30 \%$ PEG, incubating at room temperature for $5 \mathrm{~min}$, then spinning at $14000 \mathrm{rpm}$ for 15 min. The supernatant was decanted and the pellet was then rinsed two times with 500 $\mu \mathrm{l}$ 75\% ethanol, dried in SpeedVac and dissolved in $100 \mu \mathrm{l} 1 \times \mathrm{TE}$ buffer.

\subsubsection{Preparation of standards for quantitative real-time PCR}

The fungal genomic DNA was purified with phenol, and then the quality of the pure fungal DNA was checked by electrophoresis in $0.8 \%$ agarose gels. In order to determine the quantity of the DNA, densitometry values were compared with standard lambda phage DNA (methylated, from Escherichia coli host strain W3110) using Multi Analyst-Software (BioRad, Hercules, CA, USA) (Brandfass and Karlovsky 2008). 
Dilution series (0.5 pg, 1pg, 2.5 pg, 5 pg, 10 pg, 20 pg, 50 pg, 100 pg) of fungal genomic DNA were prepared to step up a standard curve in each PCR set. In addition to the standard curve 3 negative controls without template DNA (bidest water) were also processed in each PCR set. The plant samples were diluted 10 times and a PCR set was run to eliminate the matrix effect from the plant DNA which was made by comparing the q-PCR result from certain amount of fungal genomic DNA and fungal genomic DNA plus 10 times diluted cabbage DNA.

\subsubsection{Quantitative real time PCR assays}

T. harzianum specific primers (forward: 5'-TACAACTCCCAAACCCAATGTGA-3', reverse: 5'-CCGTTGTTGAAAGTTTTGATTCATTT-3') designed by Lopez-Mondejar et al. (2010) were used for the T. harzianum quantification. Quantitative PCR amplifications were performed in a total volume of $10 \mu \mathrm{l}$ using the iCycler System (CFX384 Real time system, Bio-Rad, Hercules, CA, USA). The reaction mixtures contained a final concentration of $1 \mu \mathrm{l} 10 \times$ Buffer (Bioline, Luckenwalde, Germany), $0.6 \mu \mathrm{l}$ of $50 \mathrm{mM} \mathrm{MgCl}_{2}$ (Bioline, Luckenwalde, Germany), $0.8 \mu \mathrm{l}$ of $2.5 \mathrm{mM} \mathrm{dNTP}$ (Bioline, Luckenwalde, Germany), $0.3 \mu \mathrm{l}$ of $10 \mu \mathrm{M}$ each primers (Invitrogen, Karlsruhe, Germany), $0.1 \mu \mathrm{l}$ of SybrGreen Mol Probes 1:1000 (Invitrogen, Karlsruhe, Germany), $0.02 \mu \mathrm{l}$ of DNA Polymerase (BIOTaq, Bioline, Luckenwalde, Germany), $5.88 \mu \mathrm{l}$ of sterile water and $1 \mu \mathrm{l}$ of sample DNA. The same thermal cycling conditions as Lopez-Mondejar et al. were used for amplification: started with an initial denaturation steps for 5 minutes at $95^{\circ} \mathrm{C}$, followed by 35 cycles each consisting of a denaturation step for 5 seconds at $95^{\circ} \mathrm{C}$, annealing for 15 seconds at $64{ }^{\circ} \mathrm{C}$, extension for 15 seconds at $72{ }^{\circ} \mathrm{C}$ and a plate read at the end of each cycle. This was followed by a final melting curve from $55^{\circ} \mathrm{C}$ to $95^{\circ} \mathrm{C}$ in $0.5^{\circ} \mathrm{C}$ increments held for 5 seconds at each temperature and a plate read at each temperature, which was used to determine the purity of the reaction products. 


\section{Quantification}

Two replicates for each DNA sample were assessed in PCR assay; the average data was used in the final data analysis. Since the amount of plant material and total volume of DNA volume was already known, the fungal DNA concentration in plant samples can be assessed from the PCR standard curve in each reaction.

\subsection{Feeding choice assay}

\subsubsection{No-choice feeding}

No-chocie and dual-choice experiments were performed to evaluate the impact of the T. harzianum root inoculation to the herbivore $P$. xylostella. In the no-choice feeding experiment, second instar larvae were carefully collected from the laboratory cultures. Clip cages were used to keep the larvae on the new fully-expanded leaves (three weeks after inoculation) until the larvae were reached the pupae stage. After eight days, all the larvae were successfully pupated. After then, the pupae were carefully removed from the clip cage and weighed and the consumed areas were calculated by the Digi-trace (IMATEC Elektronische Bildanalysesysteme GmbH).

\subsubsection{Dual choice feeding}

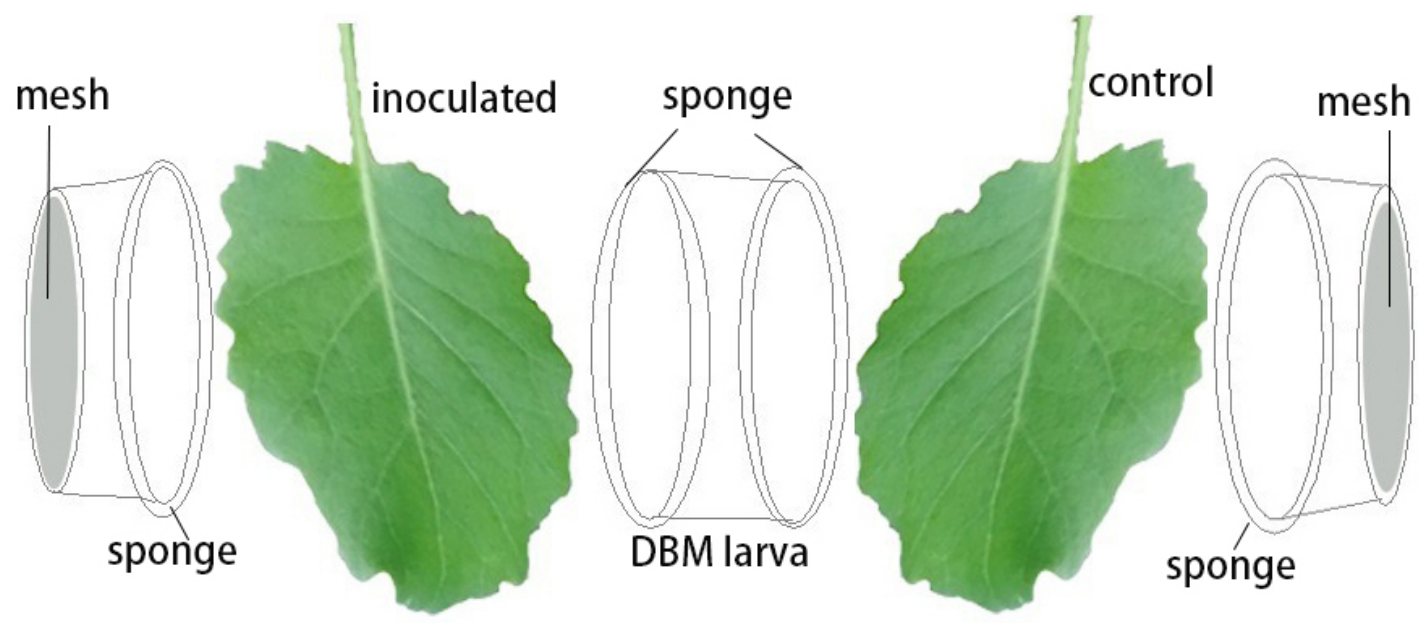




\section{Figure 1 Structure of dual-choice clip cage}

Dual-choice clip cages (diameter: $5.5 \mathrm{~cm}$ ) were used in DMB feeding choice assay. As shown in Figure 1, the dual-choice clip cage consists of three perspex cylinders; two of them have a close end with fine mesh. The feeding choice assay was processed on the undetached leaves in the same greenhouse chamber as previously mentioned. In order to protect the leaves from artificial damage, sponge was glued to the edge of the open end of the cylinder.

Three weeks after inoculation, the newly full-expanded leaves from T. harzianum inoculated and control plant were used in feeding choice assay. One second-instar DBM larvae was placed in the middle of the open cylinder, then the two leaves were attached to the clip cage with a clip carefully. After three days the consumed leaves were picked up, and the consumed area were analyzed with Digi-Trace (IMATEC Elektronische Bildanalysesysteme $\mathrm{GmbH}$ ). 20 larvae per treatment were used in this experiment.

\subsubsection{Oviposition choice assay:}

Insect rearing cages (size: $90 \mathrm{~cm} \times 50 \mathrm{~cm} \times 50 \mathrm{~cm}$ ) were used in the oviposition bioassay. Fresh pupae from lab culture were collected into a plastic box $(18 \mathrm{~cm} \times 15$ $\mathrm{cm} \times 5 \mathrm{~cm}$ ). After five days, five pairs of newly emerged male and female DBMs were placed in a test tube $(1.7 \mathrm{~cm}$ diameter $\times 10.5 \mathrm{~cm}$ length $)$ and closed off with a lid. Two cabbage plants were placed on opposite sides of the rearing cage. One plant has been inoculated with T. harzianum; the second plant was a T. harzianum-free control. To avoid the influence of the light, the cages were left in darkness for 48 hours. In a pre-experiment, the DBM was found to have a significant preference to oviposit on parafilm (Pechiney Plastic Packaging. Chicago Il), thus in this experiment, in order to enhance the oviposition performance of DBM, parafilm stripes were used to cover all the stems surface of the tested plants to form the oviposition sites. The cabbage plants were then removed from the cage and the number of the eggs oviposited on each plant 
(including the pots, stems, leaves) counted. Ten cages for each treatment were applied in the assay.

\subsection{Collection of plant volatiles}

The aboveground Volatile Organic Compounds of each three weeks old plant were collected with a modified "push-pull” method from Tholl et al. (2006). Where each plant was enclosed in a volatiles free polyester bag and the VOCs were trapped through the adsorbent tube filled with a porous polymer (Tenax ${ }^{\circledR}$ TA). The air sample was soaked in a Tenax filter with a vacuum pump with 16 volt and a 1.800 bar. Collecting time was for a period of 20 minutes. All treatments where collected at the same time under greenhouse conditions.

A Thermal Desorption System (TDS) was used for the analysis of the VOCs samples with a Hewlett Packard HP6890N gas chromatography loaded with a non-polar column $(30 \mathrm{~m} \times 0.2 \mu \mathrm{m}$ ID $\times 0.25 \mu \mathrm{m}$ film thickness, HP-5MS, Agilent Technologies, UK ) directly coupled to a quadrupole mass selective detector 5973N (Agilent Technologies, UK).

The GC-MS chromatograms were analysed using MSD ChemStation Data Analysis Software, Agilent Technologies as well as with Automated Mass Spectral Deconvolution and Identification System AMDIS. Tentative identification of the compounds was based on matching of the mass spectra with NIST 98 MS library. For each sample the peak area was calculated by integrating the peaks and the given results are based on the percentage of compound according with the total peak area.

\subsection{Statistics}

The no-choice feeding experiment data were analyzed by one way ANOVA. Percentage of dual-choice feeding area data was analyzed as paired comparison using Wilcoxon’s signed-rank test for two groups (Sokal and Rohlf 1995, Charleston et al. 
2005). For oviposition choice assay, the total number of eggs in the ten trials of the same treatments was summed and analyzed using goodness-of-fit test. The relative amount of volatile compounds in the inoculated treatments was compared with control treatments using $T$-test. All the statistics were conducted by SAS 9.3.

\section{Results}

\subsection{Selective medium re-isolation and q-PCR}

Spore germination rates of both strains were higher than $90 \%$. Nothing was growing in the medium with $100 \mu$ l third time rinsing water which indicates the sterilization was sufficient to kill the microbes on the surface of plant samples.

Table 1 Fungal recovery rate $(\%)$ of $T$. harzianum in root inoculated cabbage by selective medium re-isolation

\begin{tabular}{cccc}
\hline Treatments & Leaf $^{\mathrm{a}}$ & Stem $^{\mathrm{b}}$ & Root $^{\mathrm{c}}$ \\
\hline control & 0 & 0 & 0 \\
T39 & 0 & $43.33 \%$ & $100 \%$ \\
$\mathrm{Tu}$ & 0 & $13.33 \%$ & $100 \%$
\end{tabular}

Ten replicates per treatment. ${ }^{a}:$ three leaves per plant were obtained, after surface sterilization, four leaf discs were cut and placed on selective medium; ${ }^{\text {b: }}$ six $5 \mathrm{~mm}$ long stem segments per plant were sampled and placed on selective medium after surface sterilization; ${ }^{\mathrm{c}}$ : three $2 \mathrm{~cm}$ long root segments per plant were cut and placed on selective medium after surface sterilization; ${ }^{\mathrm{d}}: \mathrm{n}$ represents the total number of samples.

We can see from Table 1 that, with root inoculation, both of the T. harzianum strains can survive in cabbage as endophytes especially in the root as all the root samples were discovered to have fungus mycelia grown out on selective medium. Nothing was discovered from the leaf samples which indicated that the fungus did not reach the leaves three weeks after the inoculation. In the stem samples, treatment T39 (43.33\%) had a higher colonization rate than treatment $\mathrm{Tu}(13.33 \%)$.

Quantification of T. harzianum (Table 2) also confirmed the result from selective 
medium re-isolation, as a very high fungal DNA concentration was detected from the root samples while all the leaf samples were negative. Only one out of ten (T39), two out ten $(\mathrm{Tu})$ from the stem of the inoculated plants sample was positive, and the fungal concentration was also very low (see Table 2). All the root samples were positive for q- PCR. As shown in Table 2, an average $15.67 \pm 6.38$ pg DNA per mg dried root was discovered from T39 treatment, and $19.31 \pm 4.78$ pg DNA per mg dried root was found from Tu treatment.

Table 2 DNA Quantification of T. harzianum (Mean \pm SE) in root inoculated cabbage by q-PCR (pg/mg plant material) using primer pair Reverse: 5'-CCGTTGTTGAAAGTTTTGATTCATTT-3' ; 5'-TACAACTCCCAAACCCAATGTGA-3 (Lopez-Mondejar et al., 2010)

\begin{tabular}{cccc}
\hline Treatments & Leaf $^{\mathrm{a}}$ & Stem $^{\mathrm{b}}$ & Root $^{\mathrm{c}}$ \\
\hline control & $\mathrm{Nd}^{\mathrm{d}}$ & $\mathrm{Nd}$ & $\mathrm{Nd}$ \\
$\mathrm{T} 39$ & $\mathrm{Nd}$ & $0.02 \pm 0.02(1 / 10)^{\mathrm{e}}$ & $15.67 \pm 6.38(10 / 10)$ \\
$\mathrm{Tu}$ & $\mathrm{Nd}$ & $0.04 \pm 0.03(2 / 10)$ & $19.31 \pm 4.78(10 / 10)$ \\
\hline
\end{tabular}

Ten plants per treatments were used for quantification analysis; ${ }^{a}$ : number of replicates $=30$; ${ }^{b}$ : number of replicates $=10 ;{ }^{c}$ : number of replicates $=10 ;{ }^{\mathrm{d}}$ : Nd represents the amount of DNA was under the detectable amount. ${ }^{\text {e: }}$ data in the brackets followed the quantification represents the number of positive samples from the total number of tested samples. (The original data is listed in appendix)

\subsection{Behavior assay}

\subsubsection{No-choice clip cage feeding assay}

When the DBM larvae were forced to feed on leaves from different treatments, no significant differences $(p=0.85$ ) in final pupae weight were found between the inoculated and control treatments. There were also no significant difference $(p=0.71)$ in leaf consumed area between the inoculated treatments and control (Table 3). 
Table 3 No-choice feeding of $P$. xylostella on $T$. harzianum inoculated cabbage leaves (undetached)

\begin{tabular}{lccc}
\hline Treatment & Start weight $(\mathrm{mg})$ & Pupal weight $(\mathrm{mg})$ & Consumed area $\left(\mathrm{cm}^{2}\right)$ \\
\hline control & $0.74 \pm 0.06 \mathrm{a}$ & $8.21 \pm 0.29 \mathrm{a}$ & $4.27 \pm 0.29 \mathrm{a}$ \\
T39 & $0.77 \pm 0.05 \mathrm{a}$ & $8.03 \pm 0.46 \mathrm{a}$ & $4.07 \pm 0.27 \mathrm{a}$ \\
Tu & $0.72 \pm 0.06 \mathrm{a}$ & $7.92 \pm 0.30 \mathrm{a}$ & $4.47 \pm 0.18 \mathrm{a}$ \\
\hline
\end{tabular}

15 insects were tested in each treatment; means ( \pm SE) followed by the same letter within a column are not significantly different at $P>0.05$

\subsubsection{Dual-choice clip cage feeding assay}

Results in Figure 2 showed that DBM larvae preferred control plant to inoculated plant in both treatments. Wilcoxon's signed-rank test showed that the larvae had a significant preference in control plant than Tu inoculated plant $(p=0.02)$. However, although the larvae also fed on more control plant than T39 inoculated plant, the preference was not significant $(p=0.33)$.

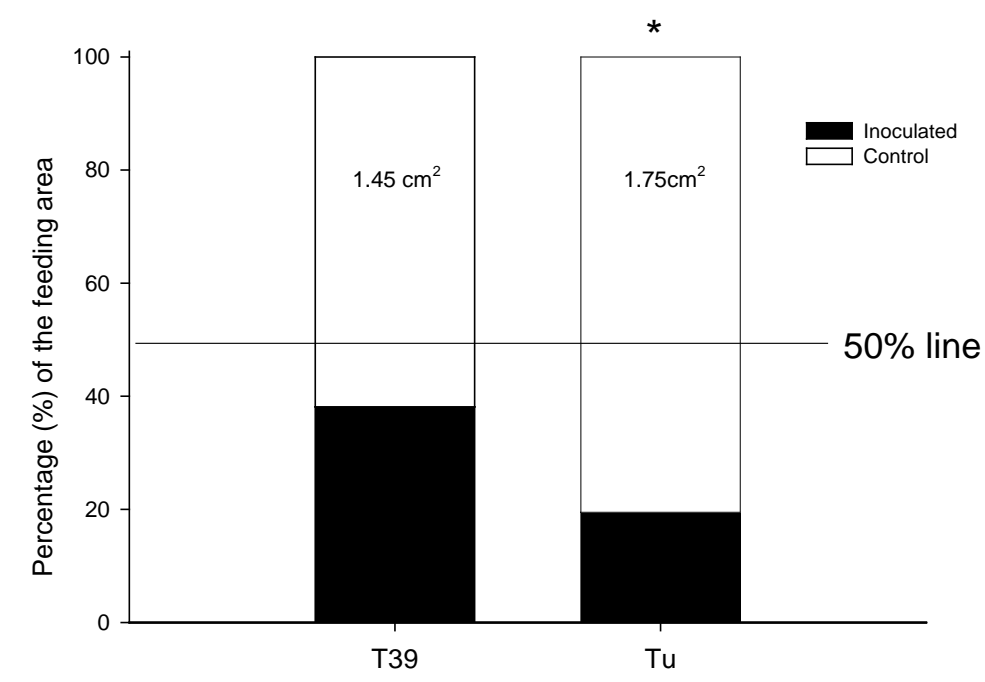

Figure2 Mean feeding area percentage of dual-choice feeding of DBM on control and T. harzianum(strain T39 and Tu) inoculated cabbage leaves (a: the mean total leaf area consumed by DBM;* represents significant feeding preference was found between $T$. harzianum inoculated and control plants (Wilcoxon's signed-rank test)) 


\subsubsection{Dual-choice oviposition assay}

T. harzianum strain T39 and Tu root inoculated cabbage plants were tested in the oviposition bioassay. We found that DBM significantly preferred the control plants over inoculated plants. In T39 inoculated trials, DBM laid an average $64.8 \pm 6.3$ eggs on control plants and $48.0 \pm 8.4$ eggs on T39 inoculated plants (goodness of fit for total number of eggs: $\mathrm{p}<0.001)$; the same pattern was discovered from the $\mathrm{Tu}$ treatments as $76.2 \pm 7.4$ eggs were found on the control plants, and $53.7 \pm 5.2$ eggs were found on treated plants (goodness of fit for total number of eggs: $\mathrm{p}<0.001$ ).

\subsection{Plant volatiles}

The volatiles profiles from $T$. harzianum inoculated plants were found to be affected by the treatment (Table 4) in the way that terpenes such as 1,8-cineole, limonene, sabinene, $\alpha$-pinene, $\beta$-pinene, $\alpha$-terpinene, $\gamma$-terpinene, $\alpha$-terpinolene, $\alpha$-thujene, $\beta$-myrcene shared a higher percentage of the relative abundance in T39 and Tu inoculated plants compared with control plants. Meanwhile, the green leaf volatiles (Z)-3-hexenyl acetate, (Z)-3-hexen-1-ol relative amount was lower in inoculated treatments than control (Table 4). 
Table 4 Compounds identified in headspaces volatiles of cabbage inoculated by Trichoderma harzianum (strain T39 and Tu)

\begin{tabular}{|c|c|c|c|}
\hline \multirow{2}{*}{ Compounds } & \multicolumn{3}{|c|}{ Relative abundance (mean \pm SE\%) } \\
\hline & Control $(\mathrm{n}=4)^{\mathrm{a}}$ & $\mathrm{Tu}(\mathrm{n}=4)$ & T39 $(n=5)$ \\
\hline \multicolumn{4}{|l|}{ Green leaf volatiles } \\
\hline (Z)-3-hexen-1-ol & $0.88 \pm 0.35(4)^{\mathrm{c}}$ & $0.42 \pm 0.16(3)$ & $0.33 \pm 0.13(4)$ \\
\hline (Z)-3-hexenyl acetate & $18.07 \pm 6.62(4)$ & $12.14 \pm 4.96(4)$ & $8.05 \pm 3.47(5)$ \\
\hline \multicolumn{4}{|l|}{ Terpenes } \\
\hline 1,8-cineole & $0.11 \pm 0.06(2)$ & $0.46 \pm 0.12(4)$ & $0.34 \pm 0.07(5) *$ \\
\hline limonene & $0.23 \pm 0.13(2)$ & $1.15 \pm 0.27(4)^{*}$ & $0.89 \pm 0.17(5) *$ \\
\hline sabinene & $0.21 \pm 0.13(2)$ & $1.00 \pm 0.39(4)$ & $0.91 \pm 0.32(5)$ \\
\hline$\alpha$-farnesene & $0.05 \pm 0.03(2)$ & $\mathrm{Nd}^{\mathrm{b}}$ & $0.10 \pm 0.05$ \\
\hline$\alpha$-pinene & $0.20 \pm 0.09$ & $0.29 \pm 0.20(3)$ & $0.48 \pm 0.07(5)$ \\
\hline$\alpha$-terpinene & $\mathrm{Nd}$ & $\mathrm{Nd}$ & $0.18 \pm 0.06(4) *$ \\
\hline$\alpha$-terpinolene & $0.06 \pm 0.04(2)$ & $0.09 \pm 0.05(2)$ & $0.08 \pm 0.01(5)$ \\
\hline$\alpha$-thujene & $0.06 \pm 0.05(1)$ & $0.14 \pm 0.12(1)$ & $0.28 \pm 0.11(4)$ \\
\hline$\beta$-myrcene & $0.22 \pm 0.05(4)$ & $0.30 \pm 0.17(3)$ & $0.36 \pm 0.06(5)$ \\
\hline$\beta$-pinene & $0.07 \pm 0.04(2)$ & $0.19 \pm 0.10(2)$ & $0.14 \pm 0.10(2)$ \\
\hline$\gamma$-terpinene & $0.03 \pm 0.03(1)$ & $0.30 \pm 0.17(2)$ & $0.25 \pm 0.04(5) * *$ \\
\hline \multicolumn{4}{|l|}{ Others } \\
\hline 1-hexyl acetate & $0.92 \pm 0.51(2)^{\mathrm{c}}$ & $0.77 \pm 0.30$ & $0.21 \pm 0.21(1)$ \\
\hline 2-ethyl-1-hexanol & $0.16 \pm 0.08(2)$ & $0.30 \pm 0.26(2)$ & $0.87 \pm 0.53(3)$ \\
\hline hexanal & $0.10 \pm 0.04$ & $0.09 \pm 0.08(2)$ & $0.05 \pm 0.05(1)$ \\
\hline 6-methyl-5-hepten-2-one & $0.08 \pm 0.04$ & $0.06 \pm 0.03(2)$ & $0.10 \pm 0.03$ \\
\hline nonanal & $0.10 \pm 0.05$ & $0.12 \pm 0.11(2)$ & $0.24 \pm 0.12$ \\
\hline (Z)-2-pentenyl acetate & $0.27 \pm 0.11(3)$ & $0.04 \pm 0.04(1)$ & $0.05 \pm 0.21(3)$ \\
\hline
\end{tabular}

${ }^{\mathrm{a}}$ :number of replications of GC-MS analysis; ${ }^{\mathrm{b}}$ : Nd represents under detective amount; ${ }^{\mathrm{c}}$ : number of replicates where the released amount of the compound was above the detection threshold by the GC-MS; * indicates $t$-test between control and inoculated treatment $0.01<p<0.05$; ** indicates $t$-test between control and inoculated treatment $p<0.01$.

\section{Discussions}

Since the re-isolation method can better describe the vitality of the fungus on inoculated plants and as a compliment the real-time quantitative PCR can demonstrate the total quantity of the fungus present in the plant, in this study, we combined both methods to evaluate the fungal endophytic colonization status. We can see that both the T39 and Tu strains could colonize the root as endophytes efficiently, but no evidence was found that this fungus could colonize the leaves, so in both of the behavior bioassays, the DBM made no direct contact to the fungus. In a 
pre-experiment conducted in $9 \mathrm{~cm}$ diameter petri dishes (unpublished data) in climate chamber $\left(24^{\circ} \mathrm{C}\right.$; $80 \%$ relative humidity; $16 \mathrm{~L}$ : 8D photoperiod), DBM larvae (started from second instar until pupated) were forced to feed on detached cabbage leaves treated with T39 and Tu $2 \times 10^{7}$ conidia/ml suspension. Compared with the control group which fed on water treated leaves, no significant difference in mortality was observed in the groups that fed on T. harzianum treated leaves. With this experiment, we can see that $T$. harzianum has no direct adverse effect on DBM larvae. In current study, we also did not found any significant difference between the DBM fed on $T$. harzianum inoculated and control plants in the no-choice experiment. In a study from Maag et al. (2014) similar results were found from the feeding experiment, as the development of DBM larvae were not affected when the host plants were grown in the presence of T. atroviride, meanwhile, the final pupal weight was also not affected, however, they did not conduct the dual-choice bioassay. In our dual-choice experiments, we found the existence of the fungi in the host plant did significantly change the preference of both the larval and adult DBM in feeding and oviposition. It was obvious from the result that, even though doesn't kill the herbivores directly, the presence of $T$. harzianum plays a role in mediating the host pant herbivore interactions.

Herbivore damage has been widely studied as an important biotic stress factor for volatile emission variation, however, only a few studies have been published in Brassicas currently. Conti et al. (2008) reported that the Murgantia histrionica feeding or oviposition significantly changed the percent emission of volatiles of the cabbage plant. They also found that, although the herbivore damage and control plants generally exhibited a similar volatile profile, with the combining of feeding and oviposition, the total terpenes content significantly decreased, the total jasmonates and total glucosinolate hydrolysis products contents significantly increased compared with the control treatment. Ibrahim et al. (2008) demonstrated that leaves infestation significantly influence the VOCs in Bt oilseed rape plants: although not always significant, in most cases the infested plants has a higher content for $\alpha$-thujene, 
sabinene, $\beta$-myrcene, 1,8-cineole, nonanal and DMNT; for the green leaf volatile, no significant differences were found in both (Z)-3-hexen-1-ol and (Z)-3-hexenyl acetate between control and infested treatments.

There are only a few studies related to the interaction of endophytic fungi colonization and headspace volatile emission until now (Battaglia et al. 2013). The opinions of the researchers are also distinct. $\mathrm{Li}$ (2008) revealed that the cabbage aphids Brevicoryne brassicae preferred control plants to than Acremoniun alternatum colonized cabbage plants. However, Jallow et al. (2008) demonstrated that the herbivore preferred the inoculated plants as with the existence of endophytic $A$. strictum, both laboratory and field strains of Helicoverpa armigera oviposited more on leaves of A. strictum inoculated plants as compared to endophyte-free plants, the existence of the endophyte also showed strong influence on the quantitative volatiles profiles analysis as most of the terpenes were found to have a lower amount in inoculated plants. Battaglia et al. (2013) found that with root colonization of $T$. longibrachiatum, the release of cis-3-hexen-1-ol, $\alpha$-pinene, methyl salicylate, longifolene, and $\beta$-caryophyllene compounds was significantly stimulated in tomato; meanwhile, the development of aphid Marcrolophus pygmaeus was also promoted. Unlike the two literatures above, in our study, DBM larvae preferred to feed on control leaves and the adults also laid more eggs on control treatments. To summarize, in our study the behavior of the herbivore was affected by the presence of the endophytic fungi.

Although the change of the VOCs under different stress has been observed in many species, to date, how plants receive volatiles and how they fortify their defenses is largely unknown (Sugimoto et al. 2014). In current study, we had lower green leaf volatile and higher terpene content in T. harzianum inoculated treatments and even though we evaluated the influence of T. harzianum inoculation on the VOCs and behavior bioassays, our experimental settings were not sufficient to establish a clear relationship between the volatile changes and altered feeding and oviposition behavior 
of DBM. With the presence of herbivores, host plants can release herbivore induced volatiles which can attract the natural enemies, therefore further impact the herbivore. Nevertheless, VOCs are not the only cues for herbivores. The Brassica pests have been documented to employ non-volatile glucosinolates as a very important host recognition cues for oviposition (de Vos et al. 2008, Mumm et al. 2008, Sun et al. 2009), furthermore, other factors like nutrition (Chen et al. 2008), color, waxes etc. also influence the herbivore behavior (Walling 2008).

Plants may defend themselves against herbivores and pathogens by direct or indirect mechanisms (Holopainen and Blande 2012). The direct defense including producing toxins, digestibility reducers, repellents and other secondary products; in addition to direct defense, plants may also defend themselves indirectly by triggering the systemic resistance (Jones and Dangl 2006) or enhancing the effectiveness of natural enemies of herbivores (Soler et al. 2007). As a well-studied fungi genus, Trichoderma spp. has been reported to elicit the systemic resistance against antagonistic fungus (although the mechanism was not clear yet). With colonization of Trichoderma spp in the host plants, many plants showed increased resistance to pathogen attack (Harman et al. 2004, Vinale et al. 2008, Shoresh et al. 2010). In a recent study, Maag et al. argued that T. atroviride strain LU132 promotes plant growth but not induce systemic resistance to DBM in oilseed rape. The study found that plant defense related compound jasmonic acid level was influenced by herbivore feeding but not by fungal treatment, moreover, the expression of the defense-related genes were also not induced by T. atroviride. Combining the fact that the DBM larvae were not affected by the presence of $T$. atroviride, the authors got the result that the $T$. atroviride strain LU132 could not induce systemic resistance to DBM in oilseed rape although it could promotes plant growth (Maag et al. 2014). We did not test the expression of the plant defense gene and the jasmonic level in our study but we found that although the presence of $T$. harzianum did not have a direct influence on the herbivore development, an indirect effect was found and confirmed by dual-choice feeding experiments. Unfortunately, the reason of this phenomenon still cannot be 
explained by this study. We have the hypothesis that the inoculation of T. harzianum may trigger the induced systemic defense which results in the change of nutrient condition and/or metabolism of the plants which further influence the behavior of herbivore behavior. However, more work related to nutrition and signal reaction pathways should be done to confirm this hypothesis.

\section{Acknowledgement}

We would like to thank Chinese Scholar Council (CSC) for the financial support.

\section{References}

Albano, S., M. Chagon, D. de Oliveira, E. Houle, P. Thibodeau, and A. Mexia. 2009. Effectiveness of Apis mellifera and Bombus impatiens as dispensers of the Rootshield ${ }^{\circledR}$ biofungicide (Trichoderma harzianum, strain T-22) in a strawberry crop. Hell Plant Prot J 2: 57-66.

Alford, D. V., C. Nilsson, and B. Ulber. 2003. Insect pests of oilseed rape crops. Biocontrol of oilseed rape pests: 9-41.

Askew, D. J., and M. D. Laing. 1993. An adapted selective medium for the quantitative isolation of Trichoderma species. Plant Pathol 42: 686-690.

Bartlet, E., M. Blight, A. Hick, and I. Williams. 1993. The responses of the cabbage seed weevil (Ceutorhynchus assimilis) to the odour of oilseed rape (Brassica napus) and to some volatile isothiocyanates. Entomol Exp Appl 68: 295-302.

Battaglia, D., S. Bossi, P. Cascone, M. C. Digilio, J. D. Prieto, P. Fanti, E. Guerrieri, L. Iodice, G. Lingua, M. Lorito, M. E. Maffei, N. Massa, M. Ruocco, R. Sasso, and V. Trotta. 2013. Tomato below ground-above fround interactions: Trichoderma longibrachiatum affects the performance of Macrosiphum euphorbiae and its natural antagonists. Mol Plant Microbe In 26: 1499-1499.

Bernays, E. A., and R. F. Chapman. 1994. Host-plant selection by phytophagous 
insects, Springer.

Brandfass, C., and P. Karlovsky. 2008. Upscaled CTAB-based DNA extraction and real-time PCR assays for Fusarium culmorum and F. graminearum DNA in plant material with reduced sampling error. Int J Mol Sci 9: 2306-2321.

Brownold, E., S. Flanders, and J. Kovach. 1997. The effect of Trichoderma harzianum on honey bee survival. NYS Fruit Project Reports Relating to IPM. Cornell Cooperative Extension, Cornell University, NYS IPM 214: 92-94.

Bruce, T. J., L. J. Wadhams, and C. M. Woodcock. 2005. Insect host location: a volatile situation. Trends in plant science 10: 269-274.

Cardoza, Y. J., K. D. Klepzig, and K. F. Raffa. 2006. Bacteria in oral secretions of an endophytic insect inhibit antagonistic fungi. Ecol Entomol 31: 636-645.

Charleston, D. S., R. Kfir, L. E. M. Vet, and M. Dicke. 2005. Behavioural responses of diamondback moth Plutella xylostella (Lepidoptera : Plutellidae) to extracts derived from Melia azedarach and Azadirachta indica. B Entomol Res 95: 457-465.

Chen, Y., J. R. Ruberson, and D. M. Olson. 2008. Nitrogen fertilization rate affects feeding, larval performance, and oviposition preference of the beet armyworm, Spodoptera exigua, on cotton. Entomol Exp Appl 126: 244-255.

Conti, E., C. Zadra, G. Salerno, B. Leombruni, D. Volpe, F. Frati, C. Marucchini, and F. Bin. 2008. Changes in the volatile profile of Brassica oleracea due to feeding and oviposition by Murgantia histrionica (Heteroptera: Pentatomidae). European Journal of Entomology 105.

De Meyer, G., J. Bigirimana, Y. Elad, and M. Hofte. 1998. Induced systemic resistance in Trichoderma harzianum T39 biocontrol of Botrytis cinerea. Eur J Plant Pathol 104: 279-286.

de Vos, M., K. L. Kriksunov, and G. Jander. 2008. Indole-3-acetonitrile production from indole glucosinolates deters oviposition by Pieris rapae. Plant physiology 146: 916-926.

Dudareva, N., F. Negre, D. A. Nagegowda, and I. Orlova. 2006. Plant volatiles: recent advances and future perspectives. Critical Reviews in Plant Sciences 25: 
417-440.

Duhl, T., D. Helmig, and A. Guenther. 2008. Sesquiterpene emissions from vegetation: a review. Biogeosciences 5.

Ebel, R. C., J. P. Mattheis, and D. A. Buchanan. 1995. Drought stress of apple trees alters leaf emissions of volatile compounds. Physiologia Plantarum 93: 709-712.

Elad, Y., and I. Chet. 1983. Improved selective media for isolation of Trichoderma spp. or Fusarium spp. Phytoparasitica 11: 55-58.

Fontana, A., M. Reichelt, S. Hempel, J. Gershenzon, and S. B. Unsicker. 2009. The effects of arbuscular mycorrhizal fungi on direct and indirect defense metabolites of Plantago lanceolata L. Journal of chemical ecology 35: 833-843.

Ganassi, S., C. Altomare, and M. Sabatini. 2009. Interactions between fungi belonging to the genus Trichoderma and Myzus persicae (Hemiptera: Aphidoidea) to open new perspectives of biologicol control. Micologia Italiana 38: 3-9.

Han, B., Z. Zhang, and Y. Fang. 2001. Electrophysiology and behavior feedback of diamondback moth, Plutella xylostella, to volatile secondary metabolites emitted by Chinese cabbage. Chinese Science Bulletin 46: 2086-2088.

Harman, G. E., C. R. Howell, A. Viterbo, I. Chet, and M. Lorito. 2004. Trichoderma species - Opportunistic, avirulent plant symbionts. Nat Rev Microbiol 2: 43-56.

Holopainen, J. K., and J. Gershenzon. 2010. Multiple stress factors and the emission of plant VOCs. Trends in plant science 15: 176-184.

Holopainen, J. K., and J. D. Blande. 2012. Molecular plant volatile communication, pp. 17-31, Sensing in nature. Springer.

Howell, C. R. 2003. Mechanisms employed by Trichoderma species in the biological control of plant diseases: The history and evolution of current concepts. Plant Dis 87: 4-10. 
Ibrahim, M., A. Stewart - Jones, J. Pulkkinen, G. Poppy, and J. Holopainen. 2008. The influence of different nutrient levels on insect - induced plant volatiles in Bt and conventional oilseed rape plants. Plant Biology 10: 97-107.

Jallow, M. F., D. Dugassa-Gobena, and S. Vidal. 2008. Influence of an endophytic fungus on host plant selection by a polyphagous moth via volatile spectrum changes. Arthropod-Plant Interactions 2: 53-62.

Jayasimha, P., and G. Henderson. 2007. Effect of Aspergillus flavus and Trichoderma harzianum on survival of Coptotermes formosanus (Isoptera : Rhinotermitidae). Sociobiology 49: 135-141.

Jones, J. D., and J. L. Dangl. 2006. The plant immune system. Nature 444: 323-329.

Kaaya, G. P., and M. A. Okech. 1990. Microorganisms associated with tsetse in nature -preliminary-results on isolation, identification and pathogenicity. Insect Sci Appl 11: 443-448.

Kovach, J., R. Petzoldt, and G. E. Harman. 2000. Use of honey bees and bumble bees to disseminate Trichoderma harzianum 1295-22 to strawberries for Botrytis control. Biological Control 18: 235-242.

Leitner, M., R. Kaiser, M. O. Rasmussen, H. Driguez, W. Boland, and A. Mithöfer. 2008. Microbial oligosaccharides differentially induce volatiles and signalling components in Medicago truncatula. Phytochemistry 69: 2029-2040.

Li, H. 2008. Preference-performance relationships in herbivorous insects feeding on oilseed rape inoculated with soil-borne fungi. Niedersächsische Staats-und Universitätsbibliothek Göttingen.

Lopez-Mondejar, R., A. Anton, S. Raidl, M. Ros, and J. A. Pascual. 2010. Quantification of the biocontrol agent Trichoderma harzianum with real-time TaqMan PCR and its potential extrapolation to the hyphal biomass. Bioresource Technol 101: 2888-2891.

Loreto, F., M. Centritto, R. Baraldi, F. Rapparini, and S. Liu. 2002. Emission of isoprenoids from natural vegetation in the Beijing region (Northern China). Plant Biosystems-An International Journal Dealing with all Aspects of Plant 
Biology 136: 251-255.

Maag, D., D. R. W. Kandula, C. Muller, A. Mendoza-Mendoza, S. D. Wratten, A. Stewart, and M. Rostas. 2014. Trichoderma atroviride LU132 promotes plant growth but not induced systemic resistance to Plutella xylostella in oilseed rape. Biocontrol 59: 241-252.

Mommaerts, V., G. Platteau, J. Boulet, G. Sterk, and G. Smagghe. 2008. Trichoderma-based biological control agents are compatible with the pollinator Bombus terrestris: A laboratory study. Biological Control 46: 463-466.

Mumm, R., M. Burow, G. Bukovinszkine'Kiss, E. Kazantzidou, U. Wittstock, M. Dicke, and J. Gershenzon. 2008. Formation of simple nitriles upon glucosinolate hydrolysis affects direct and indirect defense against the specialist herbivore, Pieris rapae. Journal of chemical ecology 34: 1311-1321.

Ownley, B. H., K. D. Gwinn, and F. E. Vega. 2010. Endophytic fungal entomopathogens with activity against plant pathogens: ecology and evolution. Biocontrol 55: 113-128.

Posada, F., and F. E. Vega. 2005. Establishment of the fungal entomopathogen Beauveria bassiana (Ascomycota : Hypocreales) as an endophyte in cocoa seedlings (Theobroma cacao). Mycologia 97: 1195-1200.

Reddy, G., and A. Guerrero. 2000. Behavioral responses of the diamondback moth, Plutella xylostella, to green leaf volatiles of Brassica oleracea Subsp. c apitata. Journal of agricultural and food chemistry 48: 6025-6029.

Renwick, J., and F. Chew. 1994. Oviposition behavior in Lepidoptera. Annual review of entomology 39: 377-400.

Santamarina, M., J. Roselló, R. Llacer, and V. Sanchis. 2002. Antagonistic activity of Penicillium oxalicum Corrie and Thom, Penicillium decumbens Thom and Trichoderma harzianum Rifai isolates against fungi, bacteria and insects in vitro. Revista iberoamericana de micología 19: 99-103.

Shakeri, J., and H. A. Foster. 2007. Proteolytic activity and antibiotic production by Trichoderma harzianum in relation to pathogenicity to insects. Enzyme 
Microb Tech 40: 961-968.

Shoresh, M., G. E. Harman, and F. Mastouri. 2010. Induced systemic resistance and plant responses to fungal biocontrol agents. Annual review of phytopathology 48: 21-43.

Sokal, R. R., and F. J. Rohlf. 1995. Biometry: the principals and practice of statistics in biological research. WH Freeman and Company, New York.

Soler, R., J. A. Harvey, A. F. Kamp, L. E. Vet, W. H. Van der Putten, N. M. Van Dam, J. F. Stuefer, R. Gols, C. A. Hordijk, and T. Martijn Bezemer. 2007. Root herbivores influence the behaviour of an aboveground parasitoid through changes in plant - volatile signals. Oikos 116: 367-376.

Sugimoto, K., K. Matsui, Y. Iijima, Y. Akakabe, S. Muramoto, R. Ozawa, M. Uefune, R. Sasaki, K. M. Alamgir, and S. Akitake. 2014. Intake and transformation to a glycoside of (Z)-3-hexenol from infested neighbors reveals a mode of plant odor reception and defense. Proceedings of the National Academy of Sciences: 201320660.

Sun, J. Y., I. E. Sønderby, B. A. Halkier, G. Jander, and M. De Vos. 2009. Non-volatile intact indole glucosinolates are host recognition cues for ovipositing Plutella xylostella. Journal of chemical ecology 35: 1427-1436.

Talekar, N. S., and A. M. Shelton. 1993. Biology, ecology, and management of the diamondback moth. Annual Review of Entomology 38: 275-301.

Tholl, D., W. Boland, A. Hansel, F. Loreto, U. S. Röse, and J. P. Schnitzler. 2006. Practical approaches to plant volatile analysis. The Plant Journal 45: 540-560.

Tingey, D. T., M. Manning, L. C. Grothaus, and W. F. Burns. 1980. Influence of light and temperature on monoterpene emission rates from slash pine. Plant Physiology 65: 797-801.

Vallat, A., H. Gu, and S. Dorn. 2005. How rainfall, relative humidity and temperature influence volatile emissions from apple trees in situ. Phytochemistry 66: 1540-1550.

Van der Steen, J., C. Langerak, C. Van Tongeren, and A. Dik. Year. Published. Aspects of the use of honeybees and bumblebees as vector of antagonistic 
micro-organisms in plant disease control, pp. 41-46. In, Proceedings of the section experimental and applied entomology-Netherlands entomological society, 2004.

Vega, F. E., P. F. Dowd, and R. J. Bartelt. 1995. Dissemination of microbial agents using an autoinoculating device and several insect species as vectors. Biological Control 5: 545-552.

Vinale, F., K. Sivasithamparam, E. L. Ghisalberti, R. Marra, S. L. Woo, and M. Lorito. 2008. Trichoderma-plant-pathogen interactions. Soil Biology and Biochemistry 40: 1-10.

Walling, L. L. 2008. Avoiding effective defenses: strategies employed by phloem-feeding insects. Plant Physiology 146: 859-866.

Wilson, D. 1995. Endophyte - the evolution of a term, and clarification of its use and definition. Oikos 73: 274-276.

Yi, H.-S., M. Heil, R. M. Adame-Álvarez, D. J. Ballhorn, and C.-M. Ryu. 2009. Airborne induction and priming of plant defenses against a bacterial pathogen. Plant physiology 151: 2152-2161.

Zalucki, M. P., A. Shabbir, R. Silva, D. Adamson, S. S. Liu, and M. J. Furlong. 2012. Estimating the economic cost of one of the world's major insect pests, Plutella xylostella (Lepidoptera: Plutellidae): just how long is a piece of string? J Econ Entomol 105: 1115-1129. 


\section{Appendix}

DNA Quantification of $T$. harzianum in root inoculated cabbage by q-PCR

\section{(pg/mg plant material) original data}

\begin{tabular}{ccccc}
\hline & \multicolumn{2}{c}{ T39 } & & Tu \\
\hline & Stem & Root & Stem & Root \\
\hline 1 & Nd & 0.943 & Nd & 18.84 \\
2 & Nd & 4.148 & Nd & 0.448 \\
3 & Nd & 26.74 & Nd & 11.01 \\
4 & Nd & 1.752 & 0.231 & 42.26 \\
5 & Nd & 4.946 & Nd & 16.82 \\
6 & Nd & 62.30 & Nd & 22.88 \\
7 & Nd & 35.44 & 0.134 & 12.64 \\
8 & 0.178 & 13.90 & Nd & 45.52 \\
9 & Nd & 5.576 & Nd & 0.640 \\
10 & Nd & 0.990 & Nd & 22.00 \\
\hline
\end{tabular}

Nd: Not detected.

No DNA was detected from all the 30 leaf samples from both T39 and Tu treatments.

No DNA was detected from all the control samples. 


\title{
Chapter 4
}

\section{Endophytic colonization of faba bean Vicia faba by two beneficial fungi: Beauveria bassiana and Trichoderma harzianum}

Leilei Zhang and Stefan Vidal

\author{
Georg-August-University Goettingen \\ Department of Crop Sciences \\ Section of Agricultural Entomology \\ Grisebachstrasse 6 \\ 37077 Goettingen Germany
}




\section{Abstract}

Root and seed inoculations were applied to introduce Beauveria bassiana (strain EABb04/01-tip (EABb04) and Naturalis) or Trichoderma harzianum (strain T39 and Tu) as endophyte in faba beans (Vicia faba). Selective medium re-isolation and quantitative PCR were used to evaluate the colonization status of the two fungi inside the host plants.

In root inoculation trials, both $B$. bassiana strains were able to colonize the root, stem and leaf. The roots had a higher colonization efficiency (EABb04: 32.5\% and Naturalis: 40.0\%) and fungal DNA concentration (EABb04: $7.11 \pm 3.20 \mathrm{pg} / \mathrm{mg}$ and Naturalis: $7.10 \pm 2.54 \mathrm{pg} / \mathrm{mg}$ ) than the stems and the leaves, which had very low colonization rates $(\leq 13.0 \%)$ and fungal DNA concentrations (over eight out of ten samples were undetectable). Both T39 and Tu strains had 100\% fungal recovery rate and a very high fungal DNA concentration $(197.67 \pm 40.29 \mathrm{pg} / \mathrm{mg}$ and $229.50 \pm 50.09$ $\mathrm{pg} / \mathrm{mg}$, respectively) in the root system. In contrast, the fungal recovery rate and concentration decreased in the stems, and subsequently nothing was found in the leaves. No significant difference of fungal DNA quantity was found between the two B. bassiana strains and the two T. harzianum strains.

In the seed inoculation trials, $B$. bassiana was discovered in the under-ground tissues (seed remains and roots), but not in the aerial parts. T. harzianum existed in all checked plant tissues (aerial parts, seed remains and roots) at a very high frequency, although the concentration in aerial parts and roots were much lower than in the seed remains. There were no significant differences in fungal DNA concentration for roots and aerial parts between the strains of both fungi; EABb04 and T39, however, had a significant higher fungal DNA concentration in the seed remains.

Key words: Beauveria bassiana, colonization, endophyte, quantitative PCR, Trichodderma harzianum 


\section{Introduction}

Endophytes are fungi or bacteria which occur inside plant tissues without causing any apparent symptom in their host plants (Wilson 1995, Abd-El-Khair et al. 2010). Several studies already demonstrated that fungal endophytes are quite common in nature and some of them are able to work as bio-control agent to defend insects, nematodes and plant pathogens (Vega et al. 2008, Biswas et al. 2012). As endophytic fungi are continuously present throughout the crop cycle, endophytic expression of the beneficial fungi within the plant system is expected to be more advantageous than an external application (Biswas et al. 2012). The fungi Beauveria bassiana Vuillemin and Trichoderma harzianum Rifai are already well-studied as endophytes in host plants, which work against both herbivores and plant pathogens.

Studies related to the most famous entomopathogen, B. bassiana, showed that this fungi was able to establish naturally as well as by artificial inoculation in many plants including maize (Bing and Lewis 1991), potato (Jones 1994), cocoa (Posada and Vega 2005), coffee (Posada et al. 2007), banana (Akello et al. 2007b), date palm (Gomez-Vidal et al. 2006), sorghum (Tefera and Vidal 2009), opium poppy (Quesada-Moraga et al. 2009), cotton, pumpkin, wheat (Gurulingappa et al. 2010), Pinus radiate (Reay et al. 2010), jute (Biswas et al. 2012) and common bean (Parsa et al. 2013). Meanwhile, the effects of endophytic B. bassiana on herbivores were also widely studied. Endophytic B. bassiana has been demonstrated to suppress many pest species: it significantly reduces the tunneling of European corn borer larvae (Ostrinia nubilalis) and successfully suppresses the stem-borer (Sesamia calamistis) in maize (Bing and Lewis 1991, Cherry et al. 2004); Moreover, B. bassiana reduces the population of banana weevil (Cosmopolites sordidus) (Akello et al. 2008)and controls the stem borer Chilo partellus in millet crop plants (Sorghum bicolor) (Reddy et al. 2009); B. bassiana has also been shown to provide systemic protection against damage by poppy stem gall wasp, Iraella luteipes in opium poppy (Quesada-Moraga et al. 2009). To date, no studies have been done to investigate whether B. bassiana is 
capable to colonize faba beans.

Trichoderma spp. are free-living fungi that are common in soil and root ecosystems. Their capacity to control plant pathogenic fungi has been widely studied (Harman et al. 2004). Direct and indirect mechanisms including mycoparasitism, antibiosis, competition, producing enzymes and inducing host plant resistance have been employed by Trichoderma spp. to defend plants against pathogenic fungi (De Meyer et al. 1998, Howell 2003). Moreover, Trichoderma spp. can also improve photosynthetic efficiency and increase nutrients uptake efficiency in plants, which result in promotion of plant growth and greater yields in crops colonized by this fungi (Shoresh et al. 2010). There is increasing evidence that many Trichoderma species are able to work in controlling the plant diseases (Wilson 1997). For example, it has been demonstrated that $T$. harzianum was able to control the damping off disease of beans (Phaseolus vulgaris) caused by Fusarium solani and Rhizoctonia solani (Abd-El-Khair et al. 2010) as well as the chocolate spot disease in faba beans caused by Botrytis fabae or B. cinerea (Saber et al. 2009, Abd El-Rahman and Mohamed 2014). Although whether the T. harzianum could also control the plant herbivore has not been ascertained, some studies indeed found T. harzianum has a negative effect for the herbivores. In recent years, several studies documented that $T$. harzianum was effective against herbivores: Santamarina et al. (2002) demonstrated a high mortality in Oncopeltus fasciatus , Cardoza et al. (2006) found a significant reduction in spruce beetle survival and reproduction, (Shakeri and Foster 2007) induced a higher mortality in Tenebrio molitor larvae and (Ganassi et al. 2009) showed a significant decreased survival of the aphid Myzus persicae. Artificial introduction would be the first step to evaluate the capability of the endophytic T. harzianum.

As an important food legume and an excellent nitrogen fixer (Sahile et al. 2010), faba beans are an important economic crop plant that is cultivated worldwide. Therefore, improving the production of this crop has become one of the objectives in agriculture in many countries (Bendahmane et al. 2012). To protect faba bean crops 
against herbivores and plant pathogens, endophytic expression of the famous bio-control fungi B. bassiana and T. harzianum can be considered as potential new efficient tools. Therefore, we use root and seed inoculation to introduce $B$. bassiana (strain: EABb04 and Naturalis) and T. harzianum (strain: T39 and Tu) into the host faba bean plants in our study. Selective medium re-isolation as well as quantitative PCR was used to confirm the existence of the endophytic fungi.

\section{Materials and methods}

\subsection{Materials}

\subsubsection{Fungi}

Table 1 Beauveria bassiana and Trichoderma harzianum strains used for endophytic establishment in faba bean

\begin{tabular}{lccc}
\hline \multicolumn{1}{c}{ species } & strains & Geographic origin & \\
\hline B. bassiana & EABb 04/01-Tip & Spain & $\begin{array}{c}\text { stem-borer Timaspis papaveris } \\
\text { (Kieffer) larva }\end{array}$ \\
& Naturalis ${ }^{2}$ & USA & $\begin{array}{c}\text { (strain ATCC74040-based } \\
\text { bioinsecticide) cotton boll weevil } \\
\text { Anthonomus grandis (Boheman) }\end{array}$ \\
T. harzianum & Tu & Thailand & Uniseeds Co. Ltd. \\
& T39 & Israel & TRICHODEX (Makhteshim-Agan \\
& & & Ltd., Tel Aviv, Israel)
\end{tabular}

Two strains of each fungal species were used in this experiment: $B$. bassiana (EABb04 (full name: EABb04/01-Tip) and Naturalis); T. harzianum (T39 and Tu). The origins and basic information on the fungal strains are listed in Table 1. Fungal conidia were harvested from 3-week-old PDA (potato dextrose agar) cultures using a camel hairbrush into a sterile beaker which had $500 \mathrm{ml}$ sterile $0.1 \%$ Tween 20 solution. The suspensions were then homogenized by using magnetic stirrer for 5 minutes and adjusted to $2 \times 10^{7}$ conidia/ml or $5 \times 10^{7}$ conidia/ml using an improved Neubauer hemocytometer. The germination of all the fungal strains was checked by PDA medium using the same method according to Posada and Vega (2005) . 


\subsubsection{Host plant}

Faba bean plants, cultivar Hangdown Grünkernig (Gevo GmbH) were used as a host. During the experiments, a soil (Fruhstorfer Erde Typ 25, Hawita Gruppe GmbH, peat fine structure with volcanic clay, pH: 5.7-6.3, Fertilization of 200-300mg N) and sand mixture (3:1 volume) was used as a growth medium. Plants were grown under controlled conditions in a greenhouse chamber $\left(24 \pm 3^{\circ} \mathrm{C}\right.$; $80 \%$ relative humidity; $16 \mathrm{~L}$ : 8D photoperiod).

\subsection{Inoculation}

\section{Root inoculation}

Faba beans were grown from seeds on a seedling bed. 10-day-old seedlings were taken out from the seedling bed, released from the root soil with tape water and inoculated with the fungal suspension. The inoculation was performed by immersing the root of the seedling into $2 \times 10^{7}$ conidia/ml spore suspension or sterile $0.1 \%$ Tween 20 solution (control treatment) for 30 minutes. Thereafter, the seedlings were transplanted into a new pot. For the root inoculation experiment, we used ten replicate pots for each fungal strain.

\section{Seed inoculation}

Faba bean seeds were surface sterilized with $2 \% \mathrm{NaClO}$ for five minutes followed by $70 \%$ ethanol for five minutes. After rinsed in sterile water for three times, the seeds were soaked in sterile water for 24 hours. The following day, the pre-treated seeds were immersed in $5 \times 10^{7}$ conidia/ml fungal suspension for two hours. The inoculated seeds were planted in pots and kept in a greenhouse chamber $\left(24 \pm 3^{\circ} \mathrm{C}\right.$; $80 \%$ relative humidity; 16L: 8D photoperiod). For the control treatment, sterilized water was used instead of the fungal suspension. Ten replicates for each treatment were used in seed inoculation. 


\subsection{Selective medium re-isolation}

Beauveria selective medium (2\% oatmeal infusion, 2\% agar, $550 \mathrm{mg} / \mathrm{L}$ dodine, 5 $\mathrm{mg} / \mathrm{L}$ chloramphenicol and $5 \mathrm{mg} / \mathrm{L}$ crystal violet; (Chase et al. 1986)) and Trichoderma selective medium (2\% agar, $2 \mathrm{~g} / \mathrm{L} \mathrm{MgSO}_{4}\left(7 \mathrm{H}_{2} \mathrm{O}\right), 0.9$ g/L K $\mathrm{HPO}_{4}, 0.15$ g/L KCl, 1.0 g/L NH${ }_{4} \mathrm{NO}_{3}, 3.0$ g/L glucose, 0.15 g/L rose bengal, 20 g/L agar, 0.25 g/L chloramphenicol, 0.2 g/L quintozene, 1.6 g/L metalaxyl (Elad and Chet 1983, Askew and Laing 1993)) were used to detect the presence of the fungi in the root inoculated plants. As the faba bean plants were quite small at the harvest time in the seed inoculated treatments, the plant material were not sufficient for both selective medium re-isolation and q-PCR, thus in these treatments, only q-PCR were used to detect the existence of the endophytic fungi.

Two weeks after the root inoculation, plants were harvested and the fungal colonization status was investigated by selective medium re-isolation. Leaf, stem and root samples were collected and surface sterilized by the following procedure: $70 \%$ ethanol for one minute, $0.5 \% \mathrm{NaClO}$ for three minutes, then samples were rinsed three times in sterile water. The samples were then surface-dried at room temperature on clean bench. For the leaf samples, ten leaf discs from each of the ten replicated plants were taken using a sterile cork borer (diameter: $8 \mathrm{~mm}$ ). For the stem samples, eight $5 \mathrm{~mm}$ stem segments were taken from each of the ten replicated plants. For the root samples, three $2 \mathrm{~cm}$ long root segments were taken from each plant, with ten replicates, a total number of 30 root samples were obtained in each treatment. The samples were cultured in $5.5 \mathrm{~cm}$ diameter petri dishes with fungi specific selective medium. In order to examine whether the surface sterilization was successful, $100 \mu \mathrm{l}$ of the third time rinsing water was pipetted and plated on the selective medium. The petri dishes were sealed with parafilm (Pechiney Plastic Packaging. Chicago Il) and incubated at $25^{\circ} \mathrm{C}$ for three weeks, by then the fungal existence was examined visually for fungal growth. Fungal growth was characterized as B. bassiana based on white dense mycelia, becoming cream to pale yellow at the edge (Humber 1997); $T$. 
harzianum based on the white, yellow, green mycelia and spores. For each treatment, the fungal percent colonization was calculated following the Petrini (1991) formula: colonization rate $=$ number of plant samples showing inoculated-fungi outgrowth divided by the total number of incubated plant samples $\times 100 \%$. The remains of the sterilized samples were well labeled and stored in $-80^{\circ} \mathrm{C}$ for molecular check.

\subsection{Quantitative real-time PCR}

Quantitative real-time PCR was used to quantify the fungal concentration in the host plant. After 17 days in the greenhouse chamber, the seed inoculated plant seedlings were harvested and surface sterilized using the same method as for the root inoculated treatments. After surface sterilization, the samples were kept separately as above ground parts, roots and seed remains. All samples (including the root inoculated samples) were stored at $-80{ }^{\circ} \mathrm{C}$ until they were freeze dried.

\subsubsection{Sample preparation}

Fungal DNA samples used for PCR assays were obtained from one-week-old potato dextrose broth (PDB) culture. Mycelium was harvested by filtration. After lyophilization, all freeze-dried fungal and plant samples were homogenized using a bead mill.

Same CTAB DNA extraction method was used for all pure fungal samples and plant samples. The steps were as follows: $50 \mathrm{mg}$ fine sample powders were transferred into $2 \mathrm{ml}$ Eppendorf-tubes, $1 \mathrm{ml}$ CTAB buffer (supplemented with $2 \mu \mathrm{l}$ mercaptoethanol and $1 \mu \mathrm{l}$ proteinase; recipe for 1 liter: $7.4448 \mathrm{~g}$ Na-EDTA; $23 \mathrm{~g}$ Sorbitol; 10 g N-Laurylsarcosine, 8 g CTAB, 47 g Sodium Chloride, 10 g Polyvinylpyrrolidon 25.000-40.000, $10 \mathrm{~mL} 1 \mathrm{M}$ Tris stock solution. $800 \mathrm{ml}$ water) was then added to each tube and mixed well; subsequently, the mixture was incubated at $42{ }^{\circ} \mathrm{C}$ for ten minutes and incubated at $65{ }^{\circ} \mathrm{C}$ for 10 minutes. In the following step, 800 $\mu$ l chloroform/isoamylalcohol (24:1) was added, mixed and incubated on ice for 
10 minutes. After spinning at 8000 rpm for 10 minutes the DNA was precipitated by transferring the supernatant into a new tube with $100 \mu \mathrm{l} 5 \mathrm{M} \mathrm{NaCl}$ and $200 \mu \mathrm{l} 30 \%$ PEG, the mixture was incubated at room temperature for 5 minutes and then spun at 14000 rpm for 15 minutes; thereafter, the supernatant was decanted and the pellet was rinsed two times with $500 \mu \mathrm{l}$ 75\% ethanol, dried in SpeedVac and dissolved in $100 \mu \mathrm{l}$ $1 \times$ TE buffer.

\subsubsection{Preparation of standards for quantitative real-time PCR}

The fungal genomic DNA was purified with phenol, and the quality of the pure fungal DNA was checked by electrophoresis in $0.8 \%$ agarose gels. In order to determine the quantity of the fungal DNA, densitometry values were compared with standard lambda phage DNA (methylated, from Escherichia coli host strain W3110) using Multi Analyst-Software (BioRad, Hercules, CA, USA) (Brandfass and Karlovsky 2008).

Dilution series (0.5 pg, 1 pg, 2.5 pg, 5 pg, 10 pg, 20 pg, 50 pg, 100 pg) of fungal genomic DNA were prepared to step up a standard curve in each PCR set. In addition to the standard curve three negative controls without template DNA were processed in each PCR set. The plant samples were diluted 25 times and a PCR set was run to eliminate the matrix effect from the plant DNA, which was made by comparing the q-PCR result from certain amount of fungal genomic DNA and fungal genomic DNA plus 25 times diluted faba bean DNA.

\subsection{3 q-PCR assays}

\section{Quantification of endophytic B. bassiana:}

Self-designed primers bsn1-2 (forward: 5'-3' GCGTCAAGGTGCTCGAAGACAG, reverse: 5'-3' TCTGGGCGGCATCCCTATTGT) were used to evaluate the concentration of Beauveria bassiana DNA in the host plant. The primers were designed based on the 1765 bp Beauveria bassiana cuticle-degrading protease 
bassiasin I (bsn1) gene (GenBank Accession No. AF154118.1). A 231 bp. product was amplified in the PCR (detailed method of primer design is described in chapter 2).

The iCycler System (CFX384 Real time system, Bio-Rad, Hercules, CA, USA) was used for the amplification and quantification of endophytic fungi. The reaction mixtures contained a final concentration of $1 \mu \mathrm{l} 10 \times$ Buffer (Bioline, Luckenwalde,

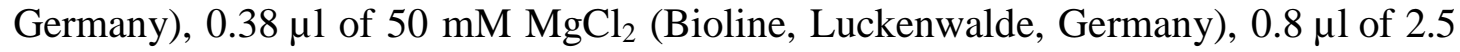
mM dNTP (Bioline, Luckenwalde, Germany), $0.3 \mu \mathrm{l}$ of $10 \mu \mathrm{M}$ each primer (Invitrogen, Karlsruhe, Germany), $0.1 \mu \mathrm{l}$ of SybrGreen Mol Probes 1:1000 (Invitrogen, Karlsruhe, Germany), $0.02 \mu$ l of DNA Polymerase (BIOTaq, Bioline, Luckenwalde, Germany), $6.1 \mu \mathrm{l}$ of sterile water and $1 \mu \mathrm{l}$ of sample DNA. The thermal cycling conditions for amplification were set as: an initial denaturation step for 5 minutes at $94^{\circ} \mathrm{C}$, followed by 35 cycles each consisting of a denaturation step for 30 seconds at $94^{\circ} \mathrm{C}$, annealing for 20 seconds at $60{ }^{\circ} \mathrm{C}$, extension for 30 seconds at $72{ }^{\circ} \mathrm{C}$ and a plate read at the end of each cycle. This was followed by a final melting curve from $55^{\circ} \mathrm{C}$ to $95^{\circ} \mathrm{C}$ in $0.5^{\circ} \mathrm{C}$ increments held for five seconds at each temperature and a plate read at each temperature, which was used to determine the purity of the products.

\section{Quantification of endophytic T. harzianum}

T. harzianum specific primers (forward: 5'-TACAACTCCCAAACCCAATGTGA-3', reverse: 5'-CCGTTGTTGAAAGTTTTGATTCATTT-3') designed by Lopez-Mondejar et al. (Lopez-Mondejar et al. 2010) were used for the T. harzianum quantification. Q-PCR amplifications were performed in a total volume of $10 \mu \mathrm{l}$. The reaction mixtures contained a final concentration of $1 \mu \mathrm{l} 10 \times$ Buffer (Bioline, Luckenwalde, Germany), $0.6 \mu \mathrm{l}$ of $50 \mathrm{mM} \mathrm{MgCl}_{2}$ (Bioline, Luckenwalde, Germany), $0.8 \mu \mathrm{l}$ of 2.5 mM dNTP (Bioline, Luckenwalde, Germany), $0.3 \mu \mathrm{l}$ of $10 \mu \mathrm{M}$ each primer (Invitrogen, Karlsruhe, Germany), $0.1 \mu$ l of SybrGreen Mol Probes 1:1000 (Invitrogen, Karlsruhe, Germany), $0.02 \mu$ l of DNA Polymerase (BIOTaq, Bioline, Luckenwalde, Germany), $5.88 \mu \mathrm{l}$ of sterile water and $1 \mu \mathrm{l}$ of sample DNA. The same thermal cycling 
conditions as Lopez-Mondejar et al. (2010) were used for amplification.

\section{Quantification}

Two replicates for each DNA sample were assessed in a PCR assay. The average data was used in the final data analysis. Since the amount of plant material and total volume of DNA volume was already known, the fungal DNA concentration in plant samples could be assessed from the PCR standard curve in each reaction. The undetected samples were considered as zero in the data analysis. The differences between the two strains were analyzed by $t$-test (SAS 9.3).

\section{Results and discussion}

Spore germination rates of both strains were higher than $90 \%$. No organism were grown on the medium with $100 \mu \mathrm{l}$ third time rinsing water indicating that the sterilization was sufficient to kill the microbes on the surface of the plant samples. It was already described by Rojo et al. (2007) that the germination of peanut seed was not affected by seed inoculation with $T$. harzianum. In this study, we also recorded that all inoculated faba bean seeds were germinated successfully. Therefore, the inoculated process of both fungi species did not harm the seed germination.

\subsection{Root inoculation}

Selective medium re-isolation and real time quantitative PCR showed that both $B$. bassiana and T. harzianum are able to establish in faba beans as endophytes via root inoculation. B. bassiana was present in all the checked tissues (Table 2 and Table 4), whereas T. harzianum was found only in stems and roots but not in leaves (Table 3 and Table 5). 
Table 2 Fungal recovery rate (\%) of $B$. bassiana in root inoculated $V$. faba by selective medium re-isolation

\begin{tabular}{lccc}
\hline Treatment & Leaf & Stem & Root \\
\hline Control & 0 & $0.0 \%$ & $0.0 \%$ \\
EABb04 & $13.0 \%$ & $12.5 \%$ & $32.5 \%$ \\
Naturalis & $8.0 \%$ & $12.5 \%$ & $40.0 \%$ \\
\hline
\end{tabular}

Ten plants (=replicates) were used in each treatment.

Table 3 Fungal recovery rate (\%) of $T$. harzianum in root inoculated $V$. faba by selective medium re-isolation

\begin{tabular}{lccc}
\hline Treatment & Leaf & Stem & Root \\
\hline Control & 0 & $0.0 \%$ & 0 \\
T39 & 0 & $13.8 \%$ & $100 \%$ \\
Tu & 0 & $16.3 \%$ & $100 \%$ \\
\hline
\end{tabular}

Ten plants (=replicates) were used in each treatment.

Via root inoculation, $B$. bassiana had high colonization rates in the root system: quantitative PCR revealed that strain EABb04 and strain Naturalis had $7.11 \pm 3.20 \mathrm{pg}$ and $7.10 \pm 2.54$ pg DNA per mg dried faba bean root, respectively (Table 4). Both strains had lower recovery rates in leafs (13.0\% and $8.0 \%)$ and stems (12.5\% in both) (Table 2), which was also confirmed by q-PCR as only one and two samples were positive in those samples, respectively (Table 4$)$. No significant differences $(p=0.996)$ in root fungal DNA concentration were found between the two B. bassiana strains (Table 4).

Table 4 DNA concentration of $B$. bassiana (mean $\pm \mathrm{SE}$ ) in root inoculated $V$. faba by q-PCR (pg/mg plant material)

\begin{tabular}{|c|c|c|c|c|c|c|}
\hline & Leaf & & Stem & & Root & \\
\hline Control & $\mathrm{Nd}^{\mathrm{a}}(0 / 10)^{\mathrm{b}}$ & & Nd $(0 / 10)$ & & $\mathrm{Nd}(0 / 10)$ & \\
\hline EABb04 & $0.23 \pm 0.23(1 / 10)$ & \multirow{2}{*}{ No data ${ }^{c}$} & $0.81 \pm 0.81(1 / 10)$ & \multirow{2}{*}{ No data } & $7.11 \pm 3.20(9 / 10)$ & \multirow{2}{*}{$p=0.996$} \\
\hline Naturalis & $1.15 \pm 0.78(2 / 10)$ & & $1.39 \pm 1.39(1 / 10)$ & & $7.10 \pm 2.54(8 / 10)$ & \\
\hline
\end{tabular}

a: Nd represents the fungal DNA concentration is below the detective amount; ${ }^{b}$ : the value in brackets represents number of positive samples in total PCR samples; ${ }^{c}: t$-test was run only in case the positive samples in both treatment were over 3 (Original data is listed in appendix Table 1). 
Table 5 DNA concentration of $T$. harzianum (mean \pm SE) in root inoculated $V$. faba by q-PCR (pg/mg plant material)

\begin{tabular}{|c|c|c|c|c|c|c|}
\hline Treatment & Lea & & Stem & & Root & \\
\hline Control & $\mathrm{Nd}^{\mathrm{a}}(0 / 10)^{\mathrm{b}}$ & & $\mathrm{Nd}(0 / 10)$ & & $\mathrm{Nd}(0 / 10)$ & \\
\hline T39 & Nd $(0 / 10)$ & \multirow{2}{*}{ No data ${ }^{\mathrm{c}}$} & $5.07 \pm 4.71(2 / 10)$ & \multirow{2}{*}{ No data } & $197.67 \pm 40.29(10 / 10)$ & \multirow{2}{*}{$p=0.637$} \\
\hline $\mathrm{Tu}$ & $\mathrm{Nd}(0 / 10)$ & & $1.25 \pm 1.10(2 / 10)$ & & $229.50 \pm 50.09(10 / 10)$ & \\
\hline
\end{tabular}

${ }^{a}$ : Nd represents the fungal DNA concentration is below the detective amount; ${ }^{\text {b}}:$ the value in brackets represents number of positive samples in total PCR samples; ' ${ }^{\mathrm{c}} t$-test was run only in case the positive samples in both treatment were over 3 (Original data is listed in appendix Table 2).

Selective medium re-isolations and q-PCR results illustrated that $T$. harzianum colonized faba bean roots perfectly (Table 3 and Table 5). The colonization rates of both strains were $100 \%$ and the q-PCR was positive for all inoculated plants; the average fungal DNA concentrations from T39 and Tu inoculated faba bean roots reached $197.67 \pm 40.29 \mathrm{pg}$ and $229.50 \pm 50.09$ pg per mg dry root, respectively. Much lower colonization rates (13.8\% for T39 and $16.3 \%$ for Tu) and fungal DNA concentrations were found in the stems, and both T. harzianum strains did not colonize the leaves (Table 5). No significant difference in T. harizanum DNA concentration was found between the two strains in the root samples (Table 5).

\subsection{Seed inoculation}

Real time quantitative PCR showed that both $B$. bassiana and T. harzianum are able to establish as endophytes in faba beans via seed inoculation (Table 6 and Table 7). B. bassiana was only present in the seed remains and roots of the 17-day-old seed inoculated faba bean seedlings, but not in aerial parts. All seed remain samples from strain EABb04 were positive for q-PCR, whereas strain Naturalis had a lower colonization frequency with half the samples (five out of ten samples) being positive. In the seed remains, the average fungal DNA concentration of strain EABb04 (42.07 \pm $12.21 \mathrm{pg} / \mathrm{mg})$ was significant higher than of strain Naturalis $(2.46 \pm 0.91 \mathrm{pg} / \mathrm{mg})(p=$ 0.010, Table 6). Colonization frequency in the root samples was generally lower than in the seed remain samples, as half the samples (five out of ten) from strain EABb04were positive for q-PCR and only two out of ten samples were positive for 
strain Naturalis (Table 6).

Table 6 DNA concentration of $B$. bassiana (mean \pm SE) in seed inoculated $V$. faba by q-PCR (pg/mg plant material)

\begin{tabular}{|c|c|c|c|c|c|c|}
\hline Treatment & Aeria & art & Root & & Seed rema & \\
\hline Control & $\mathrm{Nd}^{\mathrm{a}}(0 / 10)^{\mathrm{b}}$ & & $\mathrm{Nd}(0 / 10)$ & & Nd $(0 / 10)$ & \\
\hline EABb04 & Nd $(0 / 10)$ & \multirow{2}{*}{ No data ${ }^{c}$} & $2.51 \pm 1.08(5 / 10)$ & \multirow{2}{*}{ No data } & $42.07 \pm 12.21(10 / 10)$ & \multirow{2}{*}{$p=0.010$} \\
\hline Naturalis & Nd (0/10) & & $0.74 \pm 0.50(2 / 10)$ & & $2.46 \pm 0.91(5 / 10)$ & \\
\hline
\end{tabular}

${ }^{a}$ : Nd represents the fungal DNA concentration is below the detective amount; ${ }^{b}$ : the value in brackets represents number of positive samples in total PCR samples; ${ }^{\mathrm{c}}: t$-test was run only in case the positive samples in both treatment were over 3 (Original data is listed in appendix Table 3).

Table 7 DNA concentraionof $T$. harzianum (mean \pm SE) in seed inoculated $V$. faba by q-PCR (pg/mg plant material)

\begin{tabular}{|c|c|c|c|c|c|}
\hline Treatment & Aerial parts & Root & & Seed remains & \\
\hline Control & $\mathrm{Nd}^{\mathrm{a}}(0 / 10)^{\mathrm{b}}$ & $\mathrm{Nd}(0 / 10)$ & & Nd (0/5) & \\
\hline Т39 & $2.09 \pm 0.73(8 / 9)$ & $10.64 \pm 9.82(9 / 9)$ & \multirow{2}{*}{$p=0.683$} & $161.20 \pm 47.79(5 / 5)$ & \multirow{2}{*}{$p=0.045$} \\
\hline $\mathrm{Tu}$ & $1.02 \pm 0.57(7 / 8)$ & $6.28 \pm 3.34(8 / 8)$ & & $23.91 \pm 3.12(5 / 5)$ & \\
\hline
\end{tabular}

${ }^{a}$ : Nd represents the fungal DNA concentration is below the detective amount; ${ }^{b}$ : the value in brackets represents number of positive samples in total PCR samples. (Original data is listed in appendix Table 4).

As demonstrated in Table 7, T. harzianum could establish as an endophyte in all plant tissues of the inoculated plants. All root and seed remains samples were positive for q-PCR for both strains, and eight out of nine above-grounds samples for T39 and seven out of eight samples for Tu were positive. Strain T39 was found to have a higher fungal DNA concentration in all tissues. The highest fungal DNA concentration was found in the seed remains of the T39 inoculated treatment $(161.20 \pm 47.79 \mathrm{pg} / \mathrm{mg})$, which was significant higher than the concentration in the Tu Inoculated treatment (23.91 $\pm 3.12 \mathrm{pg} / \mathrm{mg})(p=0.045)$. In the root and aerial parts, fungal DNA was much lower with concentrations below $10.7 \mathrm{pg} / \mathrm{mg}$ dry plant material and $2.1 \mathrm{pg} / \mathrm{mg}$ dry plant material, respectively. 


\subsection{Comparison of the colonization of different fungi and different inoculation methods}

B. bassiana and T. harzianum successfully colonized faba bean plants either by root inoculation or seed inoculation, but the distribution of the endophytic fungi in the plants was not homogenous. Our studies also confirmed that with both root and seed inoculation, the colonization of both fungi in underground parts was always higher than in aerial parts. Similar results were found in cocoa and banana, as the root system has a better colonization status than other plant parts (Posada and Vega 2005, Akello et al. 2007a). The reason for the better establishment in the root might due to the fact that the fungus first establishes in the rhizosphere, and then it may colonize the root and spread to other parts of the plant. Moreover, B. bassiana as well as T. harzianum are soil borne fungi and they may be able to live and colonize in rhizosphere, thereby increasing re-infection in the roots (Akello et al. 2007a).

The colonization status of an endophyte is related to many factors. It has been illustrated that fungal species and strains, inoculation methods, host plant species and cultivar and plant growing conditions are major factors affecting the inoculation efficiency (Kessler et al. 2003, Tefera and Vidal 2009, Parsa et al. 2013). In current study, we compared the colonization efficiency of different fungal species and strains by using different inoculation methods. T. harzianum had higher colonization rates and higher fungal concentrations in all treatments and in all tissues except that it did not reach the leaves in the root inoculated treatments. The inoculation methods also affect the fungal colonization efficiency. In the root inoculated treatment, $T$. harzianum colonized the root system perfectly, but did not reach the leaves. In contratst to the root inoculation, seed inoculation with both strains of T. harzianum led to an establishment in the leaves, although the concentration was not high. T. harzianum is a strong opportunistic invader, fast grower, prolific producer of spores and also a powerful antibiotic producer with the consequence that it becomes a very successful fungus in agriculture (Monte 2001, Woo et al. 2006). Unlike T. harzianum, 
B. bassiana is a poor competitor with a slower growth and reproduction rate (Parsa et al. 2013), this might explain its lower colonization rates and fungal concentrations in most plant parts with both root and seed inoculations.

As a more economic and easier method for field applications, seed inoculation is considered to be an advanced method for introducing the endophytic fungi into the host plants. With seed inoculation, T. harzianum had a better establishment in above-ground tissues; however, we also found that both fungi had a lower efficiency in colonizing the root system via the seed inoculation than via the root inoculation method. This might due to the fact that during the inoculation section, the host faba bean carries less fungal spores at seed inoculation. Our aim is to use endophytic $B$. bassiana and T. harzianum strains to control phytopathogens and herbivorous pests. Eventhough $B$. bassiana and $T$. harzianum can survive as endophytes inside faba beans, more research is needed to improve the colonization efficiency in above-ground plant parts and to test the potential of antagonism of the inoculated plants against different herbivores and pathogens.

\section{Acknowledgement}

We would like to thank Chinese Scholar Council (CSC) for the financial support and Prof. Dr. Petr Karlovsky for his suggestions and assistances concerning the molecular experiments.

\section{References}

Abd-El-Khair, H., R. Khalifa, and K. Haggag. 2010. Effect of Trichoderma species on damping off diseases incidence, some plant enzymes activity and nutritional status of bean plants. J Am Sci 6: 486-497.

Abd El-Rahman, S., and H. Mohamed. 2014. Application of benzothiadiazole and Trichoderma harzianum to control faba bean chocolate spot disease and their 
effect on some physiological and biochemical traits. Acta Physiologiae Plantarum 36: 343-354.

Akello, J., T. Dubois, D. Coyne, and S. Kyamanywa. 2008. Endophytic Beauveria bassiana in banana (Musa spp.) reduces banana weevil (Cosmopolites sordidus) fitness and damage. Crop Prot 27: 1437-1441.

Akello, J., T. Dubois, D. Coyne, C. Gold, and S. Kyamanywa. Year. Published. Colonization and persistance of the entomopathogenic fungus, Beauveria bassiana, in tissue culture of banana, pp. 857-861. In, African Crop Science Conference Proceedings, 2007a.

Akello, J., T. Dubois, C. S. Gold, D. Coyne, J. Nakavuma, and P. Paparu. 2007b. Beauveria bassiana (Balsamo) Vuillemin as an endophyte in tissue culture banana (Musa spp.). Journal of Invertebrate Pathology 96: 34-42.

Askew, D. J., and M. D. Laing. 1993. An adapted selective medium for the quantitative isolation of Trichoderma species. Plant Pathol 42: 686-690.

Bendahmane, B. S., D. Mahiout, I. E. Benzohra, and M. Y. Benkada. 2012. Antagonism of three Trichoderma species against Botrytis fabae and $B$. cinerea, the causal agents of chocolate spot of faba bean (Vicia faba L.) In Algeria. World Applied Sciences Journal 17: 278-283.

Bing, L. A., and L. C. Lewis. 1991. Suppression of Ostrinia nubilalis (Hubner) (Lepidoptera, Pyralidae) by endophytic Beauveria bassiana (Balsamo) Vuillemin. Environ Entomol 20: 1207-1211.

Biswas, C., P. Dey, S. Satpathy, and P. Satya. 2012. Establishment of the fungal entomopathogen Beauveria bassiana as a season long endophyte in jute (Corchorus olitorius) and its rapid detection using SCAR marker. Biocontrol 57: $565-571$

Brandfass, C., and P. Karlovsky. 2008. Upscaled CTAB-based DNA extraction and real-time PCR assays for Fusarium culmorum and F. graminearum DNA in plant material with reduced sampling error. Int J Mol Sci 9: 2306-2321.

Chase, A., L. Osborne, and V. Ferguson. 1986. Selective isolation of the entomopathogenic fungi Beauveria bassiana and Metarhizium anisopliae from 
an artificial potting medim. Florida Entomologist 69: 285-292.

Cherry, A., A. Banito, D. Djegui, and C. Lomer. 2004. Suppression of the stem-borer Sesamia calamistis (Lepidoptera; Noctuidae) in maize following seed dressing, topical application and stem injection with African isolates of Beauveria bassiana. Int J Pest Manage 50: 67-73.

De Meyer, G., J. Bigirimana, Y. Elad, and M. Hofte. 1998. Induced systemic resistance in Trichoderma harzianum T39 biocontrol of Botrytis cinerea. Eur J Plant Pathol 104: 279-286.

Elad, Y., and I. Chet. 1983. Improved selective media for isolation of Trichoderma spp. or Fusarium spp. Phytoparasitica 11: 55-58.

Ganassi, S., C. Altomare, and M. Sabatini. 2009. Interactions between fungi belonging to the genus Trichoderma and Myzus persicae (Hemiptera: Aphidoidea) to open new perspectives of biologicol control. Micologia Italiana 38: 3-9.

Gomez-Vidal, S., L. V. Lopez-Llorca, H. B. Jansson, and J. Salinas. 2006. Endophytic colonization of date palm (Phoenix dactylifera L.) leaves by entomopathogenic fungi. Micron 37: 624-632.

Gurulingappa, P., G. A. Sword, G. Murdoch, and P. A. Mcgee. 2010. Colonization of crop plants by fungal entomopathogens and their effects on two insect pests when in planta. Biological Control 55: 34-41.

Harman, G. E., C. R. Howell, A. Viterbo, I. Chet, and M. Lorito. 2004. Trichoderma species - Opportunistic, avirulent plant symbionts. Nat Rev Microbiol 2: 43-56.

Howell, C. R. 2003. Mechanisms employed by Trichoderma species in the biological control of plant diseases: The history and evolution of current concepts. Plant Dis 87: 4-10.

Humber, R. A. 1997. Fungi: Identification. Manual of techniques in insect pathology: 153.

Jones, K. D. 1994. Aspects of the biology and biological control of the European corn borer in North Carolina. Ph.D. Dissertation, North Carolina State University. 
Kessler, P., H. Matzke, and S. Keller. 2003. The effect of application time and soil factors on the occurrence of Beauveria brongniartii applied as a biological control agent in soil. Journal of Invertebrate Pathology 84: 15-23.

Lopez-Mondejar, R., A. Anton, S. Raidl, M. Ros, and J. A. Pascual. 2010. Quantification of the biocontrol agent Trichoderma harzianum with real-time TaqMan PCR and its potential extrapolation to the hyphal biomass. Bioresource Technol 101: 2888-2891.

Monte, E. 2001. Understanding Trichoderma: between biotechnology and microbial ecology. International Microbiology 4: 1-4.

Parsa, S., V. Ortiz, and F. E. Vega. 2013. Establishing fungal entomopathogens as endophytes: towards endophytic biological control. Journal of visualized experiments: JoVE.

Petrini, O. 1991. Fungal endophytes of tree leaves, pp. 179-197, Microbial ecology of leaves. Springer.

Posada, F., and F. E. Vega. 2005. Establishment of the fungal entomopathogen Beauveria bassiana (Ascomycota: Hypocreales) as an endophyte in cocoa seedlings (Theobroma cacao). Mycologia 97: 1195-1200.

Posada, F., M. C. Aime, S. W. Peterson, S. A. Rehner, and F. E. Vega. 2007. Inoculation of coffee plants with the fungal entomopathogen Beauveria bassiana (Ascomycota : Hypocreales). Mycol Res 111: 748-757.

Quesada-Moraga, E., F. J. Munoz-Ledesma, and C. Santiago-Alvarez. 2009. Systemic protection of Papaver somniferum L. against Iraella luteipes (Hymenoptera: Cynipidae) by an endophytic strain of Beauveria bassiana (Ascomycota: Hypocreales). Environ Entomol 38: 723-730.

Reay, S. D., M. Brownbridge, B. Gicquel, N. J. Cummings, and T. L. Nelson. 2010. Isolation and characterization of endophytic Beauveria spp. (Ascomycota: Hypocreales) from Pinus radiata in New Zealand forests. Biological Control 54: 52-60.

Reddy, N. P., A. P. Ali Khan, U. K. Devi, H. C. Sharma, and A. Reineke. 2009. Treatment of millet crop plant ( Sorghum bicolor) with the entomopathogenic 
fungus (Beauveria bassiana) to combat infestation by the stem borer, Chilo partellus Swinhoe (Lepidoptera: Pyralidae). Journal of Asia-Pacific Entomology 12: 221-226.

Rojo, F. G., M. M. Reynoso, M. Ferez, S. N. Chulze, and A. M. Torres. 2007. Biological control by Trichoderma species of Fusarium solani causing peanut brown root rot under field conditions. Crop Prot 26: 549-555.

Saber, W., K. Abd El-Hai, and K. Ghoneem. 2009. Synergistic effect of Trichoderma and Rhizobium on both biocontrol of chocolate spot disease and induction of modulation, physiological activities and productivity of Vicia faba. Research Journal of Microbiology 4.

Sahile, S., C. Fininsa, P. Sakhuja, and S. Ahmed. 2010. Yield loss of faba bean (Vicia faba) due to chocolate spot (Botrytis fabae) in sole and mixed cropping systems in Ethiopia. Archives of Phytopathology and Plant Protection 43: 1144-1159.

Santamarina, M., J. Roselló, R. Llacer, and V. Sanchis. 2002. Antagonistic activity of Penicillium oxalicum Corrie and Thom, Penicillium decumbens Thom and Trichoderma harzianum Rifai isolates against fungi, bacteria and insects in vitro. Revista iberoamericana de micología 19: 99-103.

Shakeri, J., and H. A. Foster. 2007. Proteolytic activity and antibiotic production by Trichoderma harzianum in relation to pathogenicity to insects. Enzyme Microb Tech 40: 961-968.

Shoresh, M., G. E. Harman, and F. Mastouri. 2010. Induced systemic resistance and plant responses to fungal biocontrol agents. Annual review of phytopathology 48: 21-43.

Tefera, T., and S. Vidal. 2009. Effect of inoculation method and plant growth medium on endophytic colonization of sorghum by the entomopathogenic fungus Beauveria bassiana. Biocontrol 54: 663-669.

Vega, F. E., F. Posada, M. C. Aime, M. Pava-Ripoll, F. Infante, and S. A. Rehner. 2008. Entomopathogenic fungal endophytes. Biological Control 46: 72-82.

Wilson, D. 1995. Endophyte - the evolution of a term, and clarification of its use and 
definition. Oikos 73: 274-276.

Wilson, M. 1997. Biocontrol of aerial plant diseases in agriculture and horticulture: current approaches and future prospects. Journal of industrial Microbiology and Biotechnology 19: 188-191.

Woo, S., F. Scala, M. Ruocco, and M. Lorito. 2006. The molecular biology of the interactions between Trichoderma spp., phytopathogenic fungi, and plants. Phytopathology 96: 181-185. 


\section{Appendix}

Table 1 DNA concentration of $B$. bassiana in root inoculated $V$. faba by q-PCR (pg/mg plant material) original data

\begin{tabular}{ccc|ccc}
\hline & EABb04 & & \multicolumn{3}{c}{ Naturalis } \\
\hline leaf & stem & root & leaf & stem & root \\
\hline nd & nd & 7.26 & 4.26 & 13.94 & 18.98 \\
nd & nd & 2.80 & nd & nd & 4.96 \\
nd & 0.81 & 6.22 & nd & nd & 6.58 \\
nd & nd & nd & nd & nd & 2.44 \\
nd & nd & 2.08 & nd & nd & nd \\
nd & nd & 7.96 & nd & nd & 15.50 \\
0.23 & nd & 35.00 & nd & nd & nd \\
nd & nd & 2.62 & 6.58 & nd & 20.20 \\
nd & nd & 2.56 & nd & nd & 0.71 \\
nd & nd & 4.60 & nd & nd & 1.52 \\
\hline
\end{tabular}

nd: not detected; No DNA was detected in the control samples.

Table 2 Table 5 DNA concentration of $T$. harzianum in root inoculated $V$. faba by q-PCR (pg/mg plant material) original data

\begin{tabular}{cc|cc}
\hline & T39 & & Tu \\
\hline stem & root & stem & root \\
\hline 44.80 & 443.65 & nd & 7.02 \\
nd & 182.10 & 10.48 & 327.85 \\
nd & 56.25 & nd & 143.55 \\
nd & 344.15 & nd & 92.65 \\
nd & 309.80 & nd & 156.90 \\
nd & 64.90 & nd & 534.50 \\
nd & 124.70 & nd & 398.85 \\
0.85 & 125.70 & 1.03 & 297.85 \\
nd & 143.55 & nd & 159.00 \\
nd & 181.85 & nd & 166.85 \\
\hline
\end{tabular}

nd: not detected; No DNA was detected in the 10 leaf samples from both T39 and Tu treatments;

No DNA was detected in the control samples. 
Table 3 DNA concentration of $B$. bassiana in seed inoculated $V$. faba by q-PCR (pg/mg plant material) original data

\begin{tabular}{cc|cc}
\hline \multicolumn{2}{c|}{ EABb04 } & \multicolumn{2}{c}{ Naturalis } \\
\hline root & seed remains & root & seed remains \\
\hline 3.86 & 8.00 & nd & nd \\
3.1 & 2.34 & nd & nd \\
5.76 & 31.20 & nd & nd \\
nd & 4.00 & nd & 4.93 \\
10.29 & 106.90 & nd & 4.34 \\
nd & 83.20 & nd & nd \\
nd & 52.90 & 4.11 & nd \\
nd & 84.65 & nd & 7.53 \\
2.12 & 41.49 & nd & 5.56 \\
nd & 6.02 &
\end{tabular}

nd: not detected; No DNA was detected in the control samples and in the 10 leaf samples.

Table 4 DNA concentraionof T. harzianum in seed inoculated $V$. faba by q-PCR (pg/mg plant material) original data

\begin{tabular}{ccc|ccc}
\hline & \multicolumn{2}{c|}{ T39 } & \multicolumn{3}{c}{ Tu } \\
\hline leaf & root & seed remains & leaf & root & seed remains \\
\hline 1.73 & 0.99 & 37.50 & 0.31 & 3.42 & 26.50 \\
3.84 & 89.21 & 282.50 & 0.03 & 1.80 & 28.21 \\
3.34 & 1.06 & 250.50 & 0.24 & 2.76 & 17.90 \\
nd & 0.38 & 162.00 & 0.19 & 3.65 & 15.45 \\
2.69 & 1.10 & 73.50 & 0.13 & 4.18 & 31.80 \\
6.63 & 0.96 & - & 3.66 & 29.50 & - \\
0.08 & 0.85 & - & nd & 1.22 & - \\
0.08 & 0.92 & - & 3.61 & 3.69 & - \\
0.04 & 0.33 & - & \multicolumn{3}{|c}{ sample degraded } \\
& sample degraded & & \multicolumn{3}{|c}{ sample degraded } \\
\hline
\end{tabular}

nd: not detected; No DNA was detected in the control samples. 


\section{General discussion}

\section{Factors influence fungal endophytic establishment}

Studies already found that species and cultivar of the host plants, species and strain of endophytes, concentration of the inoculum, age and growing conditions of the host plants influence the establishment of endophytes (Kessler et al. 2003, Tefera and Vidal 2009, Parsa et al. 2013). In this study, the efficiency of different inoculation methods (leaf, root, seed, shoot and stem inoculations) to introduce the endophytic fungi $B$. bassiana and $T$. harzinaum to the host plant cabbage and faba bean was assessed.

\section{Inoculation methods and fungal species}

By summarizing all the colonization data in the four chapters we found that inoculation method plays an important role in the endophytic colonization of fungus. Moreover, the two different species B. bassiana and T. harzianum had different colonization performance by different inoculation methods. Soil is a complex and dynamic biological system; the microbial population in soil is very diverse (Nannipieri et al. 2003). One gram of soil may harbor up to 10 billion microorganisms of possibly thousands of different species (Rosselló - Mora and Amann 2001, Torsvik and Øvreås 2002).The microbes play an important role in the fungal endophytic colonization. According to whether inoculation sites touch the soil, the inoculation method can be classified as aboveground inoculation (leaf, shoot and stem), and underground inoculation (seed and root). As a strong opportunistic invader, fast growing and prolific producer of spores, and powerful antibiotic producer (Monte 2001, Woo et al. 2006), T. harzianum remains very competitive in soil. Thus in the underground inoculation treatments, $T$. harizianum was able to colonize the host plants efficiently. However, although known as a soil-borne fungus, B. bassiana grows and reproduces very slowly in soil, which makes it a poor competitor in soil 
(Parsa et al. 2013). This also explains why B. bassiana had a lower colonization rate and a lower fungal concentration in most of the plant parts with both root and seed inoculation.

\section{Host plant species}

From the results we can also get the conclusion that host plant species also influence the endophytic fungi establishment. Different plants differ in size, structure, growth rate, development time and natural endophytes. These differences may explain why endophytic fungi have a varying colonization status.

From the root inoculation data, we can see that faba bean had a higher colonization rate and higher fungal concentration than cabbage. In another failed experiment, the same seed inoculation method as described in chapter 4 was used in order to introduce B. bassiana as an endophyte in cabbage; unfortunately no positive data from re-isolation and q-PCR was found in the treated cabbages plants. The better colonization in faba bean is due to the fact that faba bean plants have bigger seeds and bigger root system. Subsequently during the inoculation, they were able to attach more fungal spores, which might form a better micro environment in the rhizosphere, help the fungi survive in the earlier competition phase and during further development.

The host plant growth rate also influences the fungal endophytic establishment. Endophytes need certain time to systemically infect the host. In the fast growing host, as the endophyte growth rate is lower than the host growth rate, the endophytic fungi content in the newly emerged tissues would decrease. This is also the reason why a higher colonization rate was always found in the inoculation site but a lower in other parts of the host.

\section{Inoculum concentration}

A proper inoculum concentration is also needed in an optimized inoculation 
procedure. Under a certain threshold, the endophytic establishment is positively correlated to the inoculum concentration; however, if the concentration is too high, the damage of the host plant caused during the inoculation might be irreversible. In two failed root inoculations, $1 \times 10^{8}$ conidia/ml B. bassiana and T. harzianum was applied to 10-day-old cabbage seedlings for 2 hours; in the next few days we found that half of the cabbage seedlings died and the rest of them had a very slow growth rate. In a high fungal concentration for a long time, the young roots of the host plants became dehydrated, which either kills the plants directly or causes irreversible damage. In the following experiments, we used older seedlings (2 weeks after transplantation with stronger roots) and adjusted the spore suspension concentration to $2 \times 10^{7}$ conidia/ml and the inoculation time to $30 \mathrm{~min}$. This resulted in no growth differences between the inoculated plants and control plants (treated with sterile water).

\section{Others}

Other factors such as fungal strains, temperature, and humidity also influence the fungal endophytic establishment. In order to optimize the fungal establishment in the host, all the factors should be considered in the inoculation procedure.

\section{Fungal endophytic colonization and insects}

The ultimate goal of this study is to develop an efficient inoculation method to introduce $B$. bassiana and $T$. harzianum into the host plant to provide durable systemic resistance against herbivory and/or disease. However, how the colonization of the endophytic fungi influence the herbivore behavior is still unclear. Plants may defend themselves against herbivores and pathogens by direct or indirect mechanisms (Holopainen and Blande 2012). The direct defense includes producing toxins, digestibility reducers, repellents and other secondary products; in addition to direct defense, plants may also defend themselves indirectly by triggering the systemic resistance (Jones and Dangl 2006) or enhancing the effectiveness of natural enemies 
of herbivores (Soler et al. 2007).

Using endophytic B. bassiana as a bio-control agent to defend herbivores has been well illustrated in many studies. In the current study (chapter 1), we also found that the colonization (leaf inoculation) of the endophytic B. bassiana significantly affected the development of the P. xylostella larvae and the oviposition choice of the adults in the bioassay. This provides the evidence that endophytic B. bassiana could serve as a potential bio-control agent. Although T. harzianum cannot kill DBM larvae directly, our study showed that, at a high colonization level, endophytic $T$. harzianum affects both the feeding choice and oviposition choice in the dual-choice bioassays,

B. bassiana has a very low level of endophytic colonization in the root inoculated trials, thus in the no-choice feeding experiment (unpublished data), although the DBM has a higher mortality, slightly longer development time and lighter pupal weight, these data were not significant; in the oviposition choice experiment, no significant difference was found between the inoculated plants and control. A similar result was observed in the oviposition choice between T. harzianum stem inoculated plants and control plants where no significant different results were found. From these experiments, we can see that the endophytic B. bassiana and $T$. harzianum significantly influence the DBM development and oviposition choice at a high colonization level. Therefore, to improve the function of endophytic fungi, an efficient inoculation method is needed.

Currently, detailed mechanisms about how inoculation influences the herbivore indirectly still remain unclear. As we found the volatile profiles were strongly influenced by endophytic T. harizanum, we have the hypothesis that the inoculation of the fungi may trigger the induced systemic defense, which results in the change of nutrient condition and/or metabolism of the plants and further influences the behavior of herbivore. However, more work related to nutrition and signal reaction pathways should be done to confirm this hypothesis. 


\section{References cited in general introduction and discussion}

Abd-El-Khair, H., R. Khalifa, and K. Haggag. 2010. Effect of Trichoderma species on damping off diseases incidence, some plant enzymes activity and nutritional status of bean plants. J Am Sci 6: 486-497.

Abd El-Rahman, S., and H. Mohamed. 2014. Application of benzothiadiazole and Trichoderma harzianum to control faba bean chocolate spot disease and their effect on some physiological and biochemical traits. Acta Physiologiae Plantarum 36: 343-354.

Akello, J., T. Dubois, D. Coyne, and S. Kyamanywa. 2008. Endophytic Beauveria bassiana in banana (Musa spp.) reduces banana weevil (Cosmopolites sordidus) fitness and damage. Crop Prot 27: 1437-1441.

Akello, J., T. Dubois, D. Coyne, and S. Kyamanywa. 2009. The effects of Beauveria bassiana dose and exposure duration on colonization and growth of tissue cultured banana Musa sp.) plants. Biological control 49: 6-10.

Akello, J., T. Dubois, D. Coyne, C. Gold, and S. Kyamanywa. Year. Published. Colonization and persistance of the entomopathogenic fungus, Beauveria bassiana, in tissue culture of banana, pp. 857-861. In, African Crop Science Conference Proceedings, 2007a.

Akello, J., T. Dubois, C. S. Gold, D. Coyne, J. Nakavuma, and P. Paparu. 2007b. Beauveria bassiana (Balsamo) Vuillemin as an endophyte in tissue culture banana (Musa spp.). Journal of Invertebrate Pathology 96: 34-42.

Alabouvette, C., C. Olivain, Q. Migheli, and C. Steinberg. 2009. Microbiological control of soil - borne phytopathogenic fungi with special emphasis on wilt inducing Fusarium oxysporum. New Phytologist 184: 529-544.

Albano, S., M. Chagon, D. de Oliveira, E. Houle, P. Thibodeau, and A. Mexia. 2009. Effectiveness of Apis mellifera and Bombus impatiens as dispensers of the Rootshield ${ }^{\circledR}$ biofungicide (Trichoderma harzianum, strain T-22) in a strawberry crop. Hell Plant Prot J 2: 57-66.

Azevedo, J. L., W. Maccheroni Jr, J. O. Pereira, and W. L. de Araújo. 2000. 
Endophytic microorganisms: a review on insect control and recent advances on tropical plants. Electronic Journal of Biotechnology 3: 15-16.

Bacon, C., J. Porter, J. Robbins, and E. Luttrell. 1977. Epichloe typhina from toxic tall fescue grasses. Applied and environmental microbiology 34: 576-581.

Bacon, C. W., J. K. Porter, and J. D. Robbins. 1975. Toxicity and occurrence of Balansia on grasses from toxic fescue pastures. Applied microbiology 29: 553-556.

Bing, L. A., and L. C. Lewis. 1991. Suppression of Ostrinia nubilalis (Hubner) (Lepidoptera, Pyralidae) by endophytic Beauveria bassiana (Balsamo) Vuillemin. Environ Entomol 20: 1207-1211.

Biswas, C., P. Dey, S. Satpathy, and P. Satya. 2012. Establishment of the fungal entomopathogen Beauveria bassiana as a season long endophyte in jute (Corchorus olitorius) and its rapid detection using SCAR marker. Biocontrol 57: 565-571.

Brownbridge, M., S. D. Reay, T. L. Nelson, and T. R. Glare. 2012. Persistence of Beauveria bassiana (Ascomycota: Hypocreales) as an endophyte following inoculation of radiata pine seed and seedlings. Biological control 61: 194-200.

Brownold, E., S. Flanders, and J. Kovach. 1997. The effect of Trichoderma harzianum on honey bee survival. NYS Fruit Project Reports Relating to IPM. Cornell Cooperative Extension, Cornell University, NYS IPM 214: 92-94.

Cardoza, Y. J., K. D. Klepzig, and K. F. Raffa. 2006. Bacteria in oral secretions of an endophytic insect inhibit antagonistic fungi. Ecol Entomol 31: 636-645.

Chase, A., L. Osborne, and V. Ferguson. 1986. Selective isolation of the entomopathogenic fungi Beauveria bassiana and Metarhizium anisopliae from an artificial potting medim. Florida Entomologist 69: 285-292.

Cherry, A., A. Banito, D. Djegui, and C. Lomer. 2004. Suppression of the stem-borer Sesamia calamistis (Lepidoptera; Noctuidae) in maize following seed dressing, topical application and stem injection with African isolates of Beauveria bassiana. Int J Pest Manage 50: 67-73.

De Bary, H. A. 1884. Vergleichende Morphologie und Biologie der Pilze Mycetozoen 
und Bacterien. Verlag von Wilhelm Engelmann Leipzig.

Ganassi, S., C. Altomare, and M. Sabatini. 2009. Interactions between fungi belonging to the genus Trichoderma and Myzus persicae (Hemiptera: Aphidoidea) to open new perspectives of biologicol control. Micologia Italiana 38: 3-9.

Gomez-Vidal, S., L. V. Lopez-Llorca, H. B. Jansson, and J. Salinas. 2006. Endophytic colonization of date palm (Phoenix dactylifera L.) leaves by entomopathogenic fungi. Micron 37: 624-632.

Gurulingappa, P., G. A. Sword, G. Murdoch, and P. A. Mcgee. 2010. Colonization of crop plants by fungal entomopathogens and their effects on two insect pests when in planta. Biological Control 55: 34-41.

Harman, G. E., C. R. Howell, A. Viterbo, I. Chet, and M. Lorito. 2004. Trichoderma species - Opportunistic, avirulent plant symbionts. Nat Rev Microbiol 2: 43-56.

Holopainen, J. K., and J. D. Blande. 2012. Molecular plant volatile communication, pp. 17-31, Sensing in nature. Springer.

Humber, R. A. 1997. Fungi: Identification. Manual of techniques in insect pathology: 153.

Jayasimha, P., and G. Henderson. 2007. Effect of Aspergillus flavus and Trichoderma harzianum on survival of Coptotermes formosanus (Isoptera : Rhinotermitidae). Sociobiology 49: 135-141.

Jones, J. D., and J. L. Dangl. 2006. The plant immune system. Nature 444: 323-329.

Jones, K. D. 1994. Aspects of the biology and biological control of the European corn borer in North Carolina. Ph.D. Dissertation, North Carolina State University.

Kessler, P., H. Matzke, and S. Keller. 2003. The effect of application time and soil factors on the occurrence of Beauveria brongniartii applied as a biological control agent in soil. Journal of Invertebrate Pathology 84: 15-23.

Kovach, J., R. Petzoldt, and G. E. Harman. 2000. Use of honey bees and bumble bees to disseminate Trichoderma harzianum 1295-22 to strawberries for Botrytis control. Biological Control 18: 235-242. 
Mommaerts, V., G. Platteau, J. Boulet, G. Sterk, and G. Smagghe. 2008. Trichoderma-based biological control agents are compatible with the pollinator Bombus terrestris: A laboratory study. Biological Control 46: 463-466.

Monte, E. 2001. Understanding Trichoderma: between biotechnology and microbial ecology. International Microbiology 4: 1-4.

Nannipieri, P., J. Ascher, M. Ceccherini, L. Landi, G. Pietramellara, and G. Renella. 2003. Microbial diversity and soil functions. European Journal of Soil Science 54: 655-670.

Parsa, S., V. Ortiz, and F. E. Vega. 2013. Establishing fungal entomopathogens as endophytes: towards endophytic biological control. Journal of visualized experiments: JoVE.

Petrini, O. 1991. Fungal endophytes of tree leaves, pp. 179-197, Microbial ecology of leaves. Springer.

Porras-Alfaro, A., and P. Bayman. 2011. Hidden fungi, emergent properties: endophytes and microbiomes. Phytopathology 49: 291.

Posada, F., and F. E. Vega. 2005. Establishment of the fungal entomopathogen Beauveria bassiana (Ascomycota: Hypocreales) as an endophyte in cocoa seedlings (Theobroma cacao). Mycologia 97: 1195-1200.

Posada, F., M. C. Aime, S. W. Peterson, S. A. Rehner, and F. E. Vega. 2007. Inoculation of coffee plants with the fungal entomopathogen Beauveria bassiana (Ascomycota : Hypocreales). Mycol Res 111: 748-757.

Posada, F. J., F. C. Chaves, T. J. Gianfagna, M. Pava-Ripoll, and P. Hebbar. 2010. Establishment of the fungal entomopathogen Beauveria bassiana as an endophyte in cocoa pods (Theobroma cacao L.). Revista UDCA actualidad \& divulgación científica 13: 71-78.

Quesada-Moraga, E., F. J. Munoz-Ledesma, and C. Santiago-Alvarez. 2009. Systemic protection of Papaver somniferum L. against Iraella luteipes (Hymenoptera: Cynipidae) by an endophytic strain of Beauveria bassiana (Ascomycota: Hypocreales). Environ Entomol 38: 723-730. 
Reay, S. D., M. Brownbridge, B. Gicquel, N. J. Cummings, and T. L. Nelson. 2010. Isolation and characterization of endophytic Beauveria spp. (Ascomycota: Hypocreales) from Pinus radiata in New Zealand forests. Biological Control 54: 52-60.

Reddy, N. P., A. P. Ali Khan, U. K. Devi, H. C. Sharma, and A. Reineke. 2009. Treatment of millet crop plant (Sorghum bicolor) with the entomopathogenic fungus ( Beauveria bassiana) to combat infestation by the stem borer, Chilo partellus Swinhoe (Lepidoptera: Pyralidae). Journal of Asia-Pacific Entomology 12: 221-226.

Rosselló - Mora, R., and R. Amann. 2001. The species concept for prokaryotes. FEMS microbiology reviews 25: 39-67.

Saber, W., K. Abd El-Hai, and K. Ghoneem. 2009. Synergistic effect of Trichoderma and Rhizobium on both biocontrol of chocolate spot disease and induction of modulation, physiological activities and productivity of Vicia faba. Research Journal of Microbiology 4.

Santamarina, M., J. Roselló, R. Llacer, and V. Sanchis. 2002. Antagonistic activity of Penicillium oxalicum Corrie and Thom, Penicillium decumbens Thom and Trichoderma harzianum Rifai isolates against fungi, bacteria and insects in vitro. Revista iberoamericana de micología 19: 99-103.

Shakeri, J., and H. A. Foster. 2007. Proteolytic activity and antibiotic production by Trichoderma harzianum in relation to pathogenicity to insects. Enzyme Microb Tech 40: 961-968.

Shoresh, M., G. E. Harman, and F. Mastouri. 2010. Induced systemic resistance and plant responses to fungal biocontrol agents. Annual review of phytopathology 48: 21-43.

Soler, R., J. A. Harvey, A. F. Kamp, L. E. Vet, W. H. Van der Putten, N. M. Van Dam, J. F. Stuefer, R. Gols, C. A. Hordijk, and T. Martijn Bezemer. 2007. Root herbivores influence the behaviour of an aboveground parasitoid through changes in plant - volatile signals. Oikos 116: 367-376.

Stone, J. K., J. Polishook, and J. White. 2004. Endophytic fungi. Biodiversity of 
Fungi. Elsevier Academic Press, Burlington: 241-270.

Tefera, T., and S. Vidal. 2009. Effect of inoculation method and plant growth medium on endophytic colonization of sorghum by the entomopathogenic fungus Beauveria bassiana. Biocontrol 54: 663-669.

Torsvik, V., and L. Øvreås. 2002. Microbial diversity and function in soil: from genes to ecosystems. Current opinion in microbiology 5: 240-245.

Van der Steen, J., C. Langerak, C. Van Tongeren, and A. Dik. Year. Published. Aspects of the use of honeybees and bumblebees as vector of antagonistic micro-organisms in plant disease control, pp. 41-46. In, Proceedings of the section experimental and applied entomology-Netherlands entomological society, 2004.

Vega, F. E., P. F. Dowd, and R. J. Bartelt. 1995. Dissemination of microbial agents using an autoinoculating device and several insect species as vectors. Biological Control 5: 545-552.

Vega, F. E., F. Posada, M. C. Aime, M. Pava-Ripoll, F. Infante, and S. A. Rehner. 2008. Entomopathogenic fungal endophytes. Biological Control 46: 72-82.

Wilson, D. 1995. Endophyte - the evolution of a term, and clarification of its use and definition. Oikos 73: 274-276.

Woo, S., F. Scala, M. Ruocco, and M. Lorito. 2006. The molecular biology of the interactions between Trichoderma spp., phytopathogenic fungi, and plants. Phytopathology 96: 181-185. 


\section{Curriculum Vitae}

Born: March, 1985, Shandong Province, China

\section{Education}

1999-2002 High school: Anqiu Experimental Middle School

2002-2006 Bachelor of Science: Ecology Department, School of Life Science, Shandong University

2007-2010 Master of Agriculture: Forestry Protection, College of Forestry, Northwest A\&F University

Advisor: Prof. Hui CHEN

Topic: Electrophysiological response of Dendroctonus armandi (Coleoptera: Curculionidae: Scolytinae) to Chines white pine volatiles 


\section{Publications}

\section{Articles}

Zhang L., Chen H., Ma C. and Tian Z.. Electrophysiological responses of Dendroctonus armandi (Coleoptera: Curculionidae: Scolytinae) to volatiles of Chinese white pine as well as to pure enantiomers and racemates of some monoterpenes. Chemoecology. 2010, Volume 20, Issue 4, pp 265-275.

\section{Posters}

Zhang L. and Vidal S. (2012) The effects of Beauveria bassiana and Trichoderma harzianum as endophytes on herbivores. Deutsche Planzenshutztagung, Braunschweig.

Zhang L. and Vidal S. (2013) Beauveria bassiana and Trichoderma harzianum as endophytes in cabbage. German Society for General and Applied Entomology (DGAAE), Göttingen, Germany. 


\section{Acknowledgement}

First of all, I have to thank my supervisor Prof. Dr. Stefan Vidal for offering me the opportunity to study here in Göttingen, for his general support, nice suggestions and encouragement during my $\mathrm{PhD}$ study.

Deeply thanks to Prof. Dr. Petr Karlovsky for his advises in molecular questions.

I would like to thank Dr. Mario Schumann, Dr. Katharina Lohaus, Sandra Aragón for their suggestions for my thesis.

Thank Mrs. Dorothea Mennerich for her excellent work in irrigating and fertilizing my plants during the last four years, thank all the colleagues of group Agriculture Entomology and group Molecular Phytopathology for their assistance and company.

Special thanks to Chinese Scholar Committee for providing me the scholarship during the last four years.

By the end, I have to thank my parents and sisters for their continuously support, encouragement and love. 